\title{
Tiling Enumeration of Hexagons with Off-Central Holes
}

\author{
Tri Lai * \\ University of Nebraska - Lincoln \\ Lincoln, NE 68588 U.S.A. \\ tlai3@unl.edu
}

Submitted: Mar 16, 2020; Accepted: Jan 25, 2022; Published: Feb 25, 2022

(C) The author. Released under the CC BY-ND license (International 4.0).

\begin{abstract}
This paper is the sequel of the author's previous paper about tiling enumerations of the cored versions of a doubly-intruded hexagon (Electron. J. Combin. 2020), in which we generalized Ciucu's work about F-cored hexagons (Adv. Math. 2017). This paper provides an extensive list of thirty tiling enumerations of hexagons with three collinear chains of triangular holes. Besides two chains of holes attaching to the boundary of the hexagon, we remove one more chain of triangles that is slightly off the center of the hexagon. Two of our enumerations imply two conjectures posed by Ciucu, Eisenkölbl, Krattenthaler, and Zare (J. Combin. Theory Ser. A 2001).
\end{abstract}

Mathematics Subject Classifications: 05A15, 05B45, 05C30

\section{Contents}

1 Introduction 1

2 Precise statement of the main result $\quad 6$

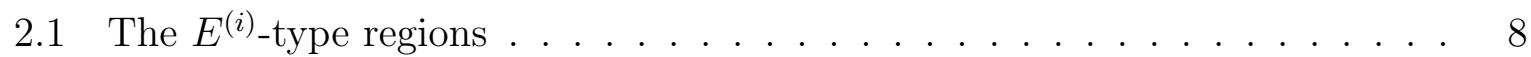

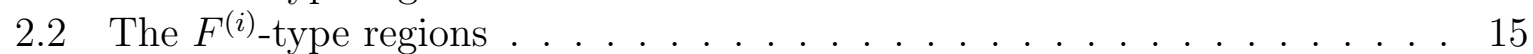

2.3 The $G^{(i)}$-type regions . . . . . . . . . . . . . . . . . . . . . . . . . . . . . . 19

2.4 Off-central counterparts of the $R^{\swarrow}$-type regions . . . . . . . . . . . 22

2.5 The $\bar{E}^{(i)}$ type regions . . . . . . . . . . . . . . . . . 26

2.6 The $\bar{F}^{(i)}$ type regions . . . . . . . . . . . . . . . . . . . . . 29

2.7 The $\bar{G}^{(i)}$ type regions . . . . . . . . . . . . . . . . . . . . . 33

2.8 The $\bar{K}^{(i)}$ type regions . . . . . . . . . . . . . . . . . . . 36

*Supported in part by Simons Foundation Collaboration Grant (\#585923). 
3 Proofs of the main theorems 39

3.1 Organization of the proof . . . . . . . . . . . . . . . . 39

3.2 Kuo condensation and other preliminary results . . . . . . . . . . . 40

3.3 Recurrences for $E^{(i)}$-type regions . . . . . . . . . . . . . . . 41

3.4 Recurrences for $F^{(i)}$-type regions . . . . . . . . . . . . . . 44

3.5 Recurrences for $G^{(i)}$-type regions . . . . . . . . . . . . . . . 47

3.6 Recurrences for $K^{(i)}$-type regions . . . . . . . . . . . . . . 50

3.7 Recurrences for $\bar{E}^{(i)}$ type regions . . . . . . . . . . . . . . . . 53

3.8 Recurrences for $\bar{F}^{(i)}$ type regions . . . . . . . . . . . . . . 55

3.9 Recurrences for $\bar{G}^{(i)}$ type regions . . . . . . . . . . . . . . . . . 57

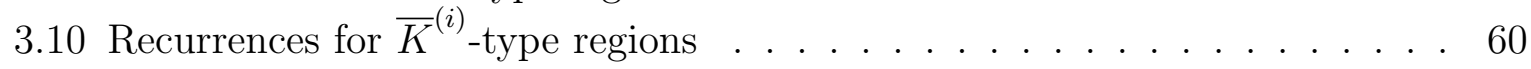

3.11 Two extremal cases for off-central regions . . . . . . . . . . . . . . . 64

3.12 Combined proof for the tiling formulas of all off-central regions . . . . . . . 67

\section{Introduction}

Motivated by MacMahon's elegant product formula for lozenge tilings a hexagon [35], James Propp posed a problem asking for a tiling formula for an almost regular hexagon with the central unit triangle removed (see Problem 2 in [38]). The problem has been solved and generalized by many authors (see, e.g., [3,6,7,10,16,31,37]). A milestone on this line of work is when Ciucu, Eisenkölbl, Krattenthaler, and Zare [7] enumerated the cored hexagon (or punctured hexagon) $C_{x, y, z}(m)$ that are obtained by removing the central equilateral triangle of side-length $m$ from the hexagon $H$ of side-lengths ${ }^{1} x, y+m$, $z, x+m, y, z+m$. We define this region in detail in the next paragraph.

We start with an auxiliary hexagon $H_{0}$ with side-lengths $x, y, z, x, y, z$ (see the hexagons with the dashed boundary in Figure 1). Next, we push the north, the northeast, and the southeast sides of the hexagon $m$ units outward, and keep other sides staying put. This way, we get a larger hexagon $H$, called the base hexagon, of side-lengths $x, y+m, z, x+m, y, z+m$. Finally, we remove an up-pointing $m$-triangle such that its left vertex is located at the closest lattice point to the center of the auxiliary hexagon $H_{0}$. We call this removed triangle a triangular hole. Precisely, there are four cases to distinguish based on the parities of $x, y, z$. When $x, y$, and $z$ have the same parity, the center of the hexagon is a lattice vertex, and our removed triangle has the left vertex at the center. One readily sees that, in this case, the triangular hole stays evenly between each pair of parallel sides of the hexagon $H$. In particular, the distance between the north side of the hexagon and the top of the triangular hole and the distance between the base of the triangular hole and the south side of the hexagon are both $\frac{y+z}{2}$; the distances corresponding to the northeast and southwest sides of the hexagon are both $\frac{x+z}{2}$; the distances corresponding to the northwest and southeast sides of the hexagon are both $\frac{x+y}{2}$ (see Figure 1(a); the hexagon with dashed boundary indicates the auxiliary hexagon). Next, we consider the case when $x$ has parity different from that of $y$ and $z$. In this case,

\footnotetext{
${ }^{1}$ From now on, we always list the side-lengths of a hexagon in clockwise order from the north side.
} 


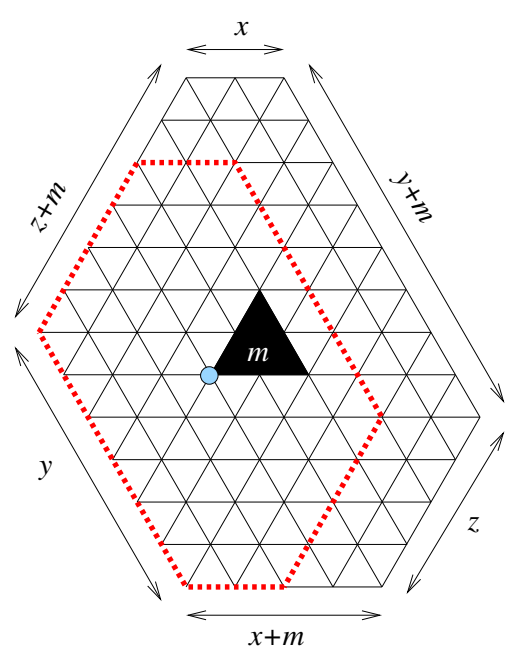

(a)

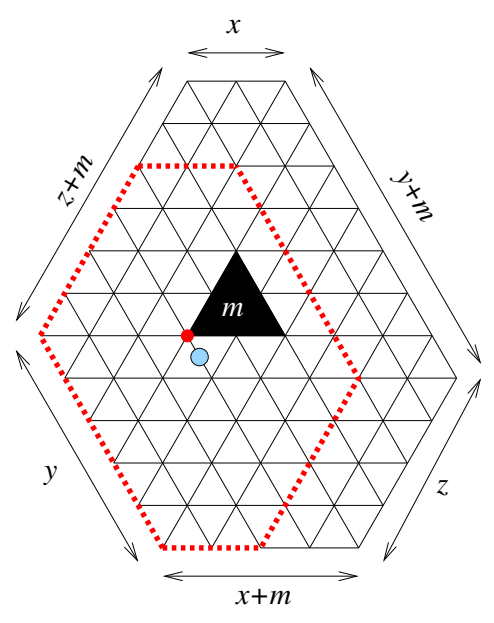

(c)

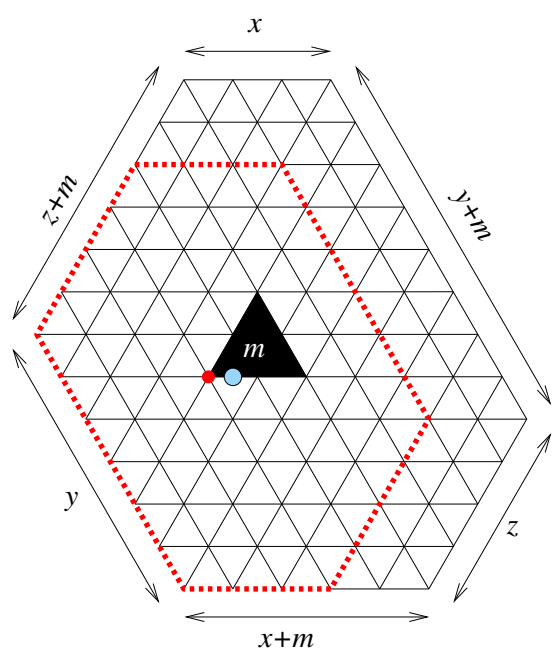

(b)

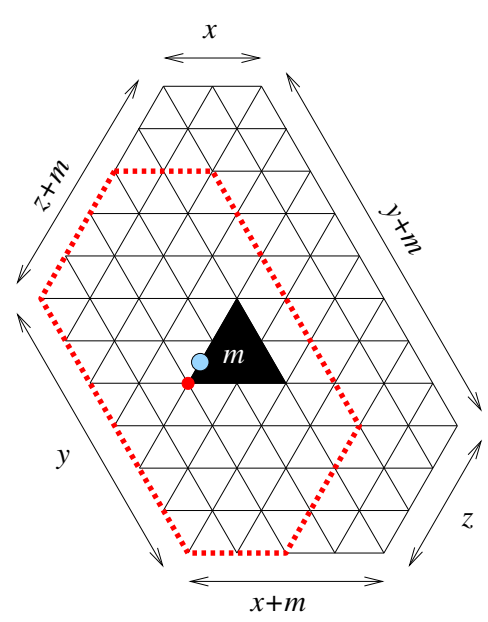

(d)

Figure 1: (a) The cored hexagon $C_{2,6,4}(2)$. (b) The cored hexagon $C_{3,6,4}(2)$. (c) The cored hexagon $C_{2,5,4}(2)$. (d) The cored hexagon $C_{2,6,3}(2)$.

the center of the auxiliary hexagon $H_{0}$ is not a lattice vertex anymore. It is the middle point of a horizontal unit lattice interval. We now place the triangular hole such that its leftmost is $1 / 2$ unit to the left of the center of the auxiliary hexagon (illustrated in Figure 1 (b); the larger shaded dot indicates the center of the auxiliary hexagon). Similarly, if $y$ has the opposite parity to $x$ and $z$, we place the triangular hole $1 / 2$ unit to the northwest of the center of the auxiliary hexagon $H_{0}$ (shown in Figure 1(c)). Finally, if $z$ has parity different from that of $x$ and $y$, the hole is located $1 / 2$ unit to the southwest of the center of $H_{0}$ (see Figure $1(\mathrm{~d})$ ). This way, we get the cored hexagon $C_{x, y, z}(m)$.

The work of Ciucu, Eisenkölbl, Krattenthaler, and Zare has been generalized further by Ciucu and Krattenthaler when the central triangular hole was extended to a cluster 
of four triangles, called a 'shamrock' in [10], and later to a chain of alternating triangles, called 'fern' by Ciucu in [6] (see, e.g., [11, 28-30] for more recent work about the fern structure). Recently, in the prequel of the paper [31], the author extended, even more, the work of Ciucu to a triple of removed ferns; besides the central fern removed, we cut off two more ferns from the boundary of the hexagon.

We note that the hole must be located in the center of the region in the above results. In the general case, if the hole is moved away from the center, the tiling formula is no longer simple. However, Ciucu, Krattenthaler, Eisenkölbl, and Zare [7] observed that when the triangular hole in the cored hexagon $C_{x, y, z}(m)$ is moved 1 unit away, the number of tilings seems to accept only several minor changes. In particular, they conjectured two striking tiling formulas for the case when $x, y, z$ have the same parity and the triangular hole is 1-unit to the left of the center (see Figure 2(a)), and the case when $x$ has parity different from that of $y$ and $z$ and the triangular hole is $3 / 2$-unit to the left of the center (see Figure 2(b)).

Next, we define the hyperfactorial function as follows:

$$
\mathrm{H}(n)= \begin{cases}\prod_{k=0}^{n-1} \Gamma(k+1) & \text { for } n \text { a positive integer; } \\ \prod_{k=0}^{n-\frac{1}{2}} \Gamma\left(k+\frac{1}{2}\right) & \text { for } n \text { a positive half-integer }\end{cases}
$$

where $\Gamma$ denotes the classical gamma function. Recall that $\Gamma(n+1)=n$ ! and $\Gamma\left(n+\frac{1}{2}\right)=$ $\frac{(2 n) !}{4^{n} n !} \sqrt{\pi}$, for a nonnegative integer $n$.

Conjecture 1 (Ciucu-Eisenkölbl-Krattenthaler-Zare; Conjecture 1 in [7]). Let $x, y, z, m$ be nonnegative integers, where $x, y, z$ have the same parity. The number of the lozenge tilings of a hexagon with side-lengths $x, y+m, z, x+m, y, z+m$, with an equilateral triangle of side-length $m$ removed at 1 unit to the left of the center, equals

$$
\begin{aligned}
\Phi_{x, y, z}(m) & :=\frac{1}{4} P_{1}(x, y, z, m) \frac{\mathrm{H}(m+x) \mathrm{H}(m+y) \mathrm{H}(m+z) \mathrm{H}(m+x+y+z)}{\mathrm{H}(m+x+y) \mathrm{H}(m+y+z) \mathrm{H}(m+z+x)} \\
& \times \frac{\mathrm{H}\left(m+\left\lfloor\frac{x+y+z}{2}\right\rfloor\right) \mathrm{H}\left(m+\left\lceil\frac{x+y+z}{2}\right\rceil\right)}{\mathrm{H}\left(m+\frac{x+y}{2}+1\right) \mathrm{H}\left(m+\frac{y+z}{2}\right) \mathrm{H}\left(m+\frac{z+x}{2}-1\right)} \\
& \times \frac{\mathrm{H}\left(\frac{m}{2}\right)^{2} \mathrm{H}\left(\left\lfloor\frac{x}{2}\right\rfloor\right) \mathrm{H}\left(\left\lceil\frac{x}{2}\right\rceil\right) \mathrm{H}\left(\left\lfloor\frac{y}{2}\right\rfloor\right) \mathrm{H}\left(\left\lceil\frac{y}{2}\right\rceil\right) \mathrm{H}\left(\left\lfloor\frac{z}{2}\right\rfloor\right) \mathrm{H}\left(\left\lceil\frac{z}{2}\right\rceil\right)}{\mathrm{H}\left(\frac{m}{2}+\left\lfloor\frac{x}{2}\right\rfloor\right) \mathrm{H}\left(\frac{m}{2}+\left\lceil\frac{x}{2}\right\rceil\right) \mathrm{H}\left(\frac{m}{2}+\left\lfloor\frac{y}{2}\right\rfloor\right) \mathrm{H}\left(\frac{m}{2}+\left\lceil\frac{y}{2}\right\rceil\right) \mathrm{H}\left(\frac{m}{2}+\left\lfloor\frac{z}{2}\right\rfloor\right) \mathrm{H}\left(\frac{m}{2}+\left\lceil\frac{z}{2}\right\rceil\right)} \\
& \times \frac{\mathrm{H}\left(\frac{m}{2}+\left\lfloor\frac{x+y}{2}\right\rfloor\right) \mathrm{H}\left(\frac{m}{2}+\left\lceil\frac{x+y}{2}\right\rceil\right) \mathrm{H}\left(\frac{m}{2}+\frac{y+z}{2}\right)^{2} \mathrm{H}\left(\frac{m}{2}+\left\lfloor\frac{z+x}{2}\right\rfloor\right) \mathrm{H}\left(\frac{m}{2}+\left\lceil\frac{z+x}{2}\right\rceil\right)}{\mathrm{H}\left(\frac{m}{2}+\left\lfloor\frac{x+y+z}{2}\right\rfloor\right) \mathrm{H}\left(\frac{m}{2}+\left\lceil\frac{x+y+z}{2}\right\rceil\right) \mathrm{H}\left(\frac{x+y}{2}-1\right) \mathrm{H}\left(\frac{y+z}{2}\right) \mathrm{H}\left(\frac{z+x}{2}+1\right)},
\end{aligned}
$$

where $P_{1}(x, y, z, m)$ is the polynomial given by

$$
P_{1}(x, y, z, m)= \begin{cases}(x+y)(x+z)+2 x m & \text { if } x \text { is even } \\ (x+y)(x+z)+2(x+y+z+m) m & \text { if } x \text { is odd }\end{cases}
$$




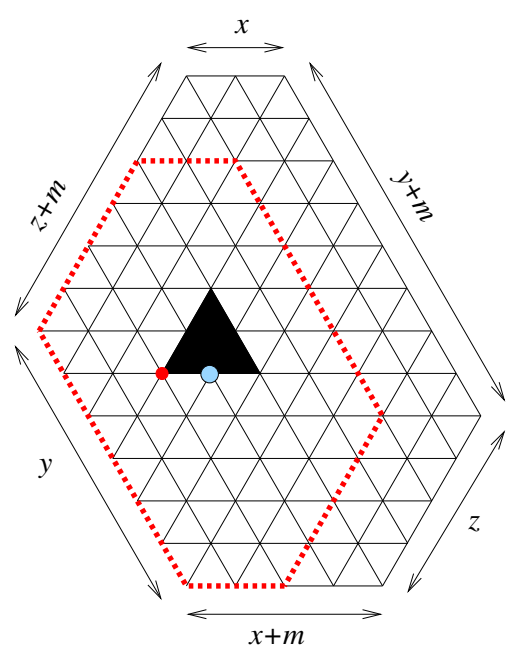

(a)

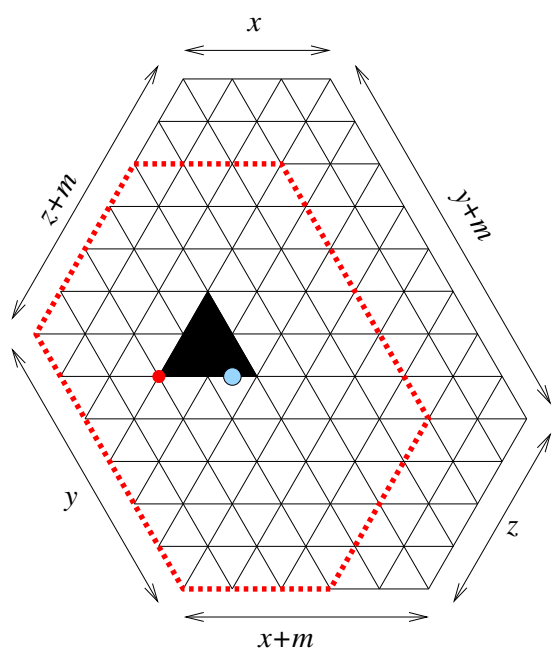

(b)

Figure 2: (a) Removal of the triangle which is off-center by one unit in the case $x, y, z$ have the same parity. (b) Removal of the triangle which is off-center by $3 / 2$ units in the case when $x$ has parity opposite to $y$ and $z$.

Conjecture 2 (Ciucu-Eisenkölbl-Krattenthaler-Zare; Conjecture 2 in [7]). Let $x, y, z, m$ be nonnegative integers, where $x$ is of parity different from the parity of $y$ and $z$. The number of the lozenge tilings of a hexagon of side-lengths $x, y+m, z, x+m, y, z+m$, with an equilateral triangle of side-length $m$ removed at $3 / 2$ unit to the left of the center, equals

$$
\begin{aligned}
\Psi_{x, z, y}(m) & :=\frac{1}{16} P_{2}(x, y, z, m) \frac{\mathrm{H}(m+x) \mathrm{H}(m+y) \mathrm{H}(m+z) \mathrm{H}(m+x+y+z)}{\mathrm{H}(m+x+y) \mathrm{H}(m+y+z) \mathrm{H}(m+z+x)} \\
& \times \frac{\mathrm{H}\left(\frac{m}{2}\right)^{2} \mathrm{H}\left(\left\lfloor\frac{x}{2}\right\rfloor\right) \mathrm{H}\left(\left\lceil\frac{x}{2}\right\rceil\right) \mathrm{H}\left(\left\lfloor\frac{y}{2}\right\rfloor\right) \mathrm{H}\left(\left\lceil\frac{y}{2}\right\rceil\right) \mathrm{H}\left(\left\lfloor\frac{z}{2}\right\rfloor\right) \mathrm{H}\left(\left\lceil\frac{z}{2}\right\rceil\right)}{\mathrm{H}\left(\frac{m}{2}+\left\lfloor\frac{x}{2}\right\rfloor\right) \mathrm{H}\left(\frac{m}{2}+\left\lceil\frac{x}{2}\right\rceil\right) \mathrm{H}\left(\frac{m}{2}+\left\lfloor\frac{y}{2}\right\rfloor\right) \mathrm{H}\left(\frac{m}{2}+\left\lceil\frac{y}{2}\right\rceil\right) \mathrm{H}\left(\frac{m}{2}+\left\lfloor\frac{z}{2}\right\rfloor\right) \mathrm{H}\left(\frac{m}{2}+\left\lceil\frac{z}{2}\right\rceil\right)} \\
& \times \frac{\mathrm{H}\left(\frac{m}{2}+\left\lfloor\frac{x+y}{2}\right\rfloor\right) \mathrm{H}\left(\frac{m}{2}+\left\lceil\frac{x+y}{2}\right\rceil\right) \mathrm{H}\left(\frac{m}{2}+\frac{y+z}{2}\right)^{2} \mathrm{H}\left(\frac{m}{2}+\left\lfloor\frac{z+x}{2}\right\rfloor\right) \mathrm{H}\left(\frac{m}{2}+\left\lceil\frac{z+x}{2}\right\rceil\right)}{\mathrm{H}\left(\frac{m}{2}+\left\lfloor\frac{x+y+z}{2}\right\rfloor\right) \mathrm{H}\left(\frac{m}{2}+\left\lceil\frac{x+y+z}{2}\right\rceil\right) \mathrm{H}\left(\left\lfloor\frac{x+y}{2}\right\rfloor-1\right) \mathrm{H}\left(\frac{y+z}{2}\right) \mathrm{H}\left(\left\lceil\frac{z+x}{2}\right\rceil+1\right)} \\
& \times \frac{\mathrm{H}\left(m+\left\lfloor\frac{x+y+z}{2}\right\rfloor\right) \mathrm{H}\left(m+\left\lceil\frac{x+y+z}{2}\right\rceil\right)}{\mathrm{H}\left(m+\left\lceil\frac{x+y}{2}\right\rceil+1\right) \mathrm{H}\left(m+\frac{y+z}{2}\right) \mathrm{H}\left(m+\left\lfloor\frac{z+x}{2}\right\rfloor-1\right)},
\end{aligned}
$$

where $P_{2}(x, y, z, m)$ is the polynomial given by

$$
\begin{aligned}
& P_{2}(x, y, z, m)= \\
& \begin{cases}\left((x+y)^{2}-1\right)\left((x+z)^{2}-1\right)+4 x m\left(x^{2}+2 x y+y^{2}+2 x z+3 y z+z^{2}\right. & \\
\left.+2 x m+3 y m+3 z m+2 m^{2}-1\right) & \text { if } x \text { is even, } \\
\left((x+y)^{2}-1\right)\left((x+z)^{2}-1\right)+4(x+y+z+m) m\left(x^{2}+x y-1\right) & \text { if } x \text { is odd. }\end{cases}
\end{aligned}
$$


The formulas (T-1) and (T-2) almost agree with the corresponding tiling formulas for cored hexagons. The only remarkable differences are the appearances of the quadratic factors $P_{1}(x, y, z, m)$ and $P_{2}(x, y, z, m)$.

One could define similarly off-central cored hexagons for the case $y$ has different parity from $x, z$ and the case $z$ has different parity from $x, y$. However, these regions could be obtained from the ones in the above conjectures by some $60^{\circ}$-rotations and will be omitted.

It is worth noticing that these conjectures were recently proved by Rosengren [43], using a different method from that in this paper. More precisely, he used lattice path combinatorics and the Selberg integral to obtain a complicated determinantal formula for the tiling generating function of a hexagon with a triangular hole at an arbitrary position. Then, by evaluating the determinant for the special cases when the triangular hole is close to the center, he was able to verify Ciucu-Eisenkölbl-Krattenthaler-Zare's Conjectures 1 and 2 .

Inspired by the above two conjectures and the previous work in [31], we would like to investigate similar situations for the case of hexagons with three ferns removed. Intuitively, we remove a fern next to the center of the hexagon. Then we remove two more ferns at the same level such that the new ferns are touching the boundary of the hexagons. Based on the parity of the side-lengths of our hexagons and the inner fern's position, there are thirty different regions to enumerate. One may be surprised that the number of situations needed to be considered is significantly larger than that in the case of cored hexagons. The reason is that, unlike the cored hexagons, $60^{\circ}$-rotations do not preserve the structures of our regions.

The rest of the paper is organized as follows. Section 2 carefully defines all 30 offcentral regions and states their exact tiling enumerations. Section 3 quotes several fundamental results in the enumeration of tilings, especially two versions of Kuo's graphical condensation [21], that will be the key to our proof. Section 4 is devoted to the detailed proof of our main theorems.

\section{Precise statement of the main result}

Recall that in the prequel paper [31], we enumerated eight different families of hexagons with three ferns removed (one fern is removed from the center, and two more ferns are removed from the boundary). These families are divided into two groups based on the relative position of the lattice line $l$, which contains the three ferns and the west and east vertices of the hexagon. In particular, we have four ' $R$-families,' $R^{\odot}, R^{\leftarrow}, R^{\nwarrow}$, and $R^{\swarrow}$, in the case when $l$ separates the east and west vertices of the hexagon, and four 'Q-families,' $Q^{\odot}, Q^{\leftarrow}, Q^{\nwarrow}$, and $Q^{\nearrow}$, in the case when $l$ leaves both east and west vertices of the hexagon on the same side (see detailed definition in Section 2 of [31]).

Each of the above eight families of regions has several off-central counterparts that will be defined carefully in the following eight subsections.

In order to state our next tilling enumerations, we define five more functions, besides the functions $\Phi$ and $\Psi$ in Conjectures 1 and 2, as follows. 
We define the functions $\Theta_{x, y, z}(m)$ and $\Theta_{x, y, z}^{\prime}(m)$ by:

$$
\begin{aligned}
\Theta_{x, y, z}(m) & :=Q_{1}(y, z, m) \cdot \frac{\mathrm{H}(m+x) \mathrm{H}(m+y) \mathrm{H}(m+z) \mathrm{H}(m+x+y+z)}{\mathrm{H}(m+x+y) \mathrm{H}(m+y+z) \mathrm{H}(m+z+x)} \\
& \times \frac{\mathrm{H}\left(m+\left\lfloor\frac{x+y+z}{2}\right\rfloor\right) \mathrm{H}\left(m+\left\lceil\frac{x+y+z}{2}\right\rceil\right)}{\mathrm{H}\left(m+\left\lfloor\frac{x+y}{2}\right\rfloor\right) \mathrm{H}\left(m+\frac{y+z}{2}+1\right) \mathrm{H}\left(m+\left\lfloor\frac{z+x}{2}\right\rfloor\right)} \\
& \times \frac{\mathrm{H}\left(\frac{m}{2}\right)^{2} \mathrm{H}\left(\left\lfloor\frac{x}{2}\right\rfloor\right) \mathrm{H}\left(\left\lceil\frac{x}{2}\right\rceil\right) \mathrm{H}\left(\left\lfloor\frac{y}{2}\right\rfloor\right) \mathrm{H}\left(\left\lceil\frac{y}{2}\right\rceil\right) \mathrm{H}\left(\left\lfloor\frac{z}{2}\right\rfloor\right) \mathrm{H}\left(\left\lceil\frac{z}{2}\right\rceil\right)}{\mathrm{H}\left(\frac{m}{2}+\left\lfloor\frac{x}{2}\right\rfloor\right) \mathrm{H}\left(\frac{m}{2}+\left\lceil\frac{x}{2}\right\rceil\right) \mathrm{H}\left(\frac{m}{2}+\left\lfloor\frac{y}{2}\right\rfloor\right) \mathrm{H}\left(\frac{m}{2}+\left\lceil\frac{y}{2}\right\rceil\right) \mathrm{H}\left(\frac{m}{2}+\left\lfloor\frac{z}{2}\right\rfloor\right) \mathrm{H}\left(\frac{m}{2}+\left\lceil\frac{z}{2}\right\rceil\right)} \\
& \times \frac{\mathrm{H}\left(\frac{m}{2}+\left\lfloor\frac{x+y}{2}\right\rfloor\right) \mathrm{H}\left(\frac{m}{2}+\left\lceil\frac{x+y}{2}\right\rceil\right) \mathrm{H}\left(\frac{m}{2}+\frac{y+z}{2}\right)^{2} \mathrm{H}\left(\frac{m}{2}+\left\lfloor\frac{z+x}{2}\right\rfloor\right) \mathrm{H}\left(\frac{m}{2}+\left\lceil\frac{z+x}{2}\right\rceil\right)}{\mathrm{H}\left(\frac{m}{2}+\left\lfloor\frac{x+y+z}{2}\right\rfloor\right) \mathrm{H}\left(\frac{m}{2}+\left\lceil\frac{x+y+z}{2}\right\rceil\right) \mathrm{H}\left(\left\lceil\frac{x+y}{2}\right\rceil\right) \mathrm{H}\left(\frac{y+z}{2}\right) \mathrm{H}\left(\left\lceil\frac{z+x}{2}\right\rceil\right)}
\end{aligned}
$$

and

$$
\begin{aligned}
\Theta_{x, y, z}^{\prime}(m) & :=Q_{1}(y, z, m) \cdot \frac{\mathrm{H}(m+x) \mathrm{H}(m+y) \mathrm{H}(m+z) \mathrm{H}(m+x+y+z)}{\mathrm{H}(m+x+y) \mathrm{H}(m+y+z) \mathrm{H}(m+z+x)} \\
& \times \frac{\mathrm{H}\left(m+\left\lfloor\frac{x+y+z}{2}\right\rfloor\right) \mathrm{H}\left(m+\left\lceil\frac{x+y+z}{2}\right\rceil\right)}{\mathrm{H}\left(m+\left\lceil\frac{x+y}{2}\right\rceil\right) \mathrm{H}\left(m+\frac{y+z}{2}-1\right) \mathrm{H}\left(m+\left\lceil\frac{z+x}{2}\right\rceil\right)} \\
& \times \frac{\mathrm{H}\left(\frac{m}{2}\right)^{2} \mathrm{H}\left(\left\lfloor\frac{x}{2}\right\rfloor\right) \mathrm{H}\left(\left\lceil\frac{x}{2}\right\rceil\right) \mathrm{H}\left(\left\lfloor\frac{y}{2}\right\rfloor\right) \mathrm{H}\left(\left\lceil\frac{y}{2}\right\rceil\right) \mathrm{H}\left(\left\lfloor\frac{z}{2}\right\rfloor\right) \mathrm{H}\left(\left\lceil\frac{z}{2}\right\rceil\right)}{\mathrm{H}\left(\frac{m}{2}+\left\lfloor\frac{x}{2}\right\rfloor\right) \mathrm{H}\left(\frac{m}{2}+\left\lceil\frac{x}{2}\right\rceil\right) \mathrm{H}\left(\frac{m}{2}+\left\lfloor\frac{y}{2}\right\rfloor\right) \mathrm{H}\left(\frac{m}{2}+\left\lceil\frac{y}{2}\right\rceil\right) \mathrm{H}\left(\frac{m}{2}+\left\lfloor\frac{z}{2}\right\rfloor\right) \mathrm{H}\left(\frac{m}{2}+\left\lceil\frac{z}{2}\right\rceil\right)} \\
& \times \frac{\mathrm{H}\left(\frac{m}{2}+\left\lfloor\frac{x+y}{2}\right\rfloor\right) \mathrm{H}\left(\frac{m}{2}+\left\lceil\frac{x+y}{2}\right\rceil\right) \mathrm{H}\left(\frac{m}{2}+\frac{y+z}{2}\right)^{2} \mathrm{H}\left(\frac{m}{2}+\left\lfloor\frac{z+x}{2}\right\rfloor\right) \mathrm{H}\left(\frac{m}{2}+\left\lceil\frac{z+x}{2}\right\rceil\right)}{\mathrm{H}\left(\frac{m}{2}+\left\lfloor\frac{x+y+z}{2}\right\rfloor\right) \mathrm{H}\left(\frac{m}{2}+\left\lceil\frac{x+y+z}{2}\right\rceil\right) \mathrm{H}\left(\left\lfloor\frac{x+y}{2}\right\rfloor\right) \mathrm{H}\left(\frac{y+z}{2}+1\right) \mathrm{H}\left(\left\lfloor\frac{z+x}{2}\right\rfloor\right)},
\end{aligned}
$$

where $Q_{1}(y, z, m)$ is $m+\frac{y+z}{2}$ if $x$ is even, and is $\frac{y+z}{2}$ if $x$ is odd. One readily sees that the $\Theta$ - and $\Theta^{\prime}$-functions are almost the same, except for certain differences at several hyperfactorial factors.

We next define the function $\Lambda_{x, y, z}(m)$ and its variation $\Lambda_{x, y, z}^{\prime}(m)$ by:

$$
\begin{aligned}
\Lambda_{x, y, z}(m):= & \frac{1}{8} Q_{2}(x, y, z, m) \cdot \frac{\mathrm{H}(m+x) \mathrm{H}(m+y) \mathrm{H}(m+z) \mathrm{H}(m+x+y+z)}{\mathrm{H}(m+x+y) \mathrm{H}(m+y+z) \mathrm{H}(m+z+x)} \\
& \times \frac{\mathrm{H}\left(m+\left\lfloor\frac{x+y+z}{2}\right\rfloor\right) \mathrm{H}\left(m+\left\lceil\frac{x+y+z}{2}\right\rceil\right)}{\mathrm{H}\left(m+\left\lceil\frac{x+y}{2}\right\rceil\right) \mathrm{H}\left(m+\frac{y+z}{2}+1\right) \mathrm{H}\left(m+\left\lfloor\frac{z+x}{2}\right\rfloor-1\right)} \\
& \times \frac{\mathrm{H}\left(\frac{m}{2}\right)^{2} \mathrm{H}\left(\left\lfloor\frac{x}{2}\right\rfloor\right) \mathrm{H}\left(\left\lceil\frac{x}{2}\right\rceil\right) \mathrm{H}\left(\left\lfloor\frac{y}{2}\right\rfloor\right) \mathrm{H}\left(\left\lceil\frac{y}{2}\right\rceil\right) \mathrm{H}\left(\left\lfloor\frac{z}{2}\right\rfloor\right) \mathrm{H}\left(\left\lceil\frac{z}{2}\right\rceil\right)}{\mathrm{H}\left(\frac{m}{2}+\left\lfloor\frac{x}{2}\right\rfloor\right) \mathrm{H}\left(\frac{m}{2}+\left\lceil\frac{x}{2}\right\rceil\right) \mathrm{H}\left(\frac{m}{2}+\left\lfloor\frac{y}{2}\right\rfloor\right) \mathrm{H}\left(\frac{m}{2}+\left\lceil\frac{y}{2}\right\rceil\right) \mathrm{H}\left(\frac{m}{2}+\left\lfloor\frac{z}{2}\right\rfloor\right) \mathrm{H}\left(\frac{m}{2}+\left\lceil\frac{z}{2}\right\rceil\right)} \\
& \times \frac{\mathrm{H}\left(\frac{m}{2}+\left\lfloor\frac{x+y}{2}\right\rfloor\right) \mathrm{H}\left(\frac{m}{2}+\left\lceil\frac{x+y}{2}\right\rceil\right) \mathrm{H}\left(\frac{m}{2}+\frac{y+z}{2}\right)^{2} \mathrm{H}\left(\frac{m}{2}+\left\lfloor\frac{z+x}{2}\right\rfloor\right) \mathrm{H}\left(\frac{m}{2}+\left\lceil\frac{z+x}{2}\right\rceil\right)}{\mathrm{H}\left(\frac{m}{2}+\left\lfloor\frac{x+y+z}{2}\right\rfloor\right) \mathrm{H}\left(\frac{m}{2}+\left\lceil\frac{x+y+z}{2}\right\rceil\right) \mathrm{H}\left(\left\lfloor\frac{x+y}{2}\right\rfloor\right) \mathrm{H}\left(\frac{y+z}{2}-1\right) \mathrm{H}\left(\left\lceil\frac{z+x}{2}\right\rceil+1\right)}
\end{aligned}
$$

and

$$
\Lambda_{x, y, z}^{\prime}(m):=\frac{1}{8} Q_{2}(x, y, z, m) \cdot \frac{\mathrm{H}(m+x) \mathrm{H}(m+y) \mathrm{H}(m+z) \mathrm{H}(m+x+y+z)}{\mathrm{H}(m+x+y) \mathrm{H}(m+y+z) \mathrm{H}(m+z+x)}
$$




$$
\begin{aligned}
& \times \frac{\mathrm{H}\left(m+\left\lfloor\frac{x+y+z}{2}\right\rfloor\right) \mathrm{H}\left(m+\left\lceil\frac{x+y+z}{2}\right\rceil\right)}{\mathrm{H}\left(m+\left\lfloor\frac{x+y}{2}\right\rfloor\right) \mathrm{H}\left(m+\frac{y+z}{2}-1\right) \mathrm{H}\left(m+\left\lceil\frac{z+x}{2}\right\rceil+1\right)} \\
& \times \frac{\mathrm{H}\left(\frac{m}{2}\right)^{2} \mathrm{H}\left(\left\lfloor\frac{x}{2}\right\rfloor\right) \mathrm{H}\left(\left\lceil\frac{x}{2}\right\rceil\right) \mathrm{H}\left(\left\lfloor\frac{y}{2}\right\rfloor\right) \mathrm{H}\left(\left\lceil\frac{y}{2}\right\rceil\right) \mathrm{H}\left(\left\lfloor\frac{z}{2}\right\rfloor\right) \mathrm{H}\left(\left\lceil\frac{z}{2}\right\rceil\right)}{\mathrm{H}\left(\frac{m}{2}+\left\lfloor\frac{x}{2}\right\rfloor\right) \mathrm{H}\left(\frac{m}{2}+\left\lceil\frac{x}{2}\right\rceil\right) \mathrm{H}\left(\frac{m}{2}+\left\lfloor\frac{y}{2}\right\rfloor\right) \mathrm{H}\left(\frac{m}{2}+\left\lceil\frac{y}{2}\right\rceil\right) \mathrm{H}\left(\frac{m}{2}+\left\lfloor\frac{z}{2}\right\rfloor\right) \mathrm{H}\left(\frac{m}{2}+\left\lceil\frac{z}{2}\right\rceil\right)} \\
& \times \frac{\mathrm{H}\left(\frac{m}{2}+\left\lfloor\frac{x+y}{2}\right\rfloor\right) \mathrm{H}\left(\frac{m}{2}+\left\lceil\frac{x+y}{2}\right\rceil\right) \mathrm{H}\left(\frac{m}{2}+\frac{y+z}{2}\right)^{2} \mathrm{H}\left(\frac{m}{2}+\left\lfloor\frac{z+x}{2}\right\rfloor\right) \mathrm{H}\left(\frac{m}{2}+\left\lceil\frac{z+x}{2}\right\rceil\right)}{\mathrm{H}\left(\frac{m}{2}+\left\lfloor\frac{x+y+z}{2}\right\rfloor\right) \mathrm{H}\left(\frac{m}{2}+\left\lceil\frac{x+y+z}{2}\right\rceil\right) \mathrm{H}\left(\left\lceil\frac{x+y}{2}\right\rceil\right) \mathrm{H}\left(\frac{y+z}{2}+1\right) \mathrm{H}\left(\left\lfloor\frac{z+x}{2}\right\rfloor-1\right)},
\end{aligned}
$$

in which the polynomial $Q_{2}(x, y, z, m)$ is defined as

$$
\begin{aligned}
& Q_{2}(x, y, z, m) \\
& \quad=\left\{\begin{array}{cl}
(x+z+2 m-1)((z+1)(x+z-1)+2 x(m-1)) & \text { if } x \text { is even } \\
+(y+1)\left((x+z)^{2}+4 x m-1\right) & \text { if } x \text { is odd } \\
z\left((x+z+2 m)^{2}-1\right)+y\left((x+z+2 m)^{2}-4 m(x+m)-1\right)
\end{array}\right.
\end{aligned}
$$

Finally, we define a counterpart of the function $\Psi$ in Conjecture 2:

$$
\begin{aligned}
\Psi_{x, y, z}^{\prime}(m):= & \frac{1}{16} \frac{\mathrm{H}(m+x) \mathrm{H}(m+y) \mathrm{H}(m+z) \mathrm{H}(m+x+y+z)}{\mathrm{H}(m+x+y) \mathrm{H}(m+y+z) \mathrm{H}(m+z+x)} \\
& \times \frac{\mathrm{H}\left(\frac{m}{2}\right)^{2} \mathrm{H}\left(\left\lfloor\frac{x}{2}\right\rfloor\right) \mathrm{H}\left(\left\lceil\frac{x}{2}\right\rceil\right) \mathrm{H}\left(\left\lfloor\frac{y}{2}\right\rfloor\right) \mathrm{H}\left(\left\lceil\frac{y}{2}\right\rceil\right) \mathrm{H}\left(\left\lfloor\frac{z}{2}\right\rfloor\right) \mathrm{H}\left(\left\lceil\frac{z}{2}\right\rceil\right)}{\mathrm{H}\left(\frac{m}{2}+\left\lfloor\frac{x}{2}\right\rfloor\right) \mathrm{H}\left(\frac{m}{2}+\left\lceil\frac{x}{2}\right\rceil\right) \mathrm{H}\left(\frac{m}{2}+\left\lfloor\frac{y}{2}\right\rfloor\right) \mathrm{H}\left(\frac{m}{2}+\left\lceil\frac{y}{2}\right\rceil\right) \mathrm{H}\left(\frac{m}{2}+\left\lfloor\frac{z}{2}\right\rfloor\right) \mathrm{H}\left(\frac{m}{2}+\left\lceil\frac{z}{2}\right\rceil\right)} \\
& \times \frac{\mathrm{H}\left(\frac{m}{2}+\left\lfloor\frac{x+y}{2}\right\rfloor\right) \mathrm{H}\left(\frac{m}{2}+\left\lceil\frac{x+y}{2}\right\rceil\right) \mathrm{H}\left(\frac{m}{2}+\frac{y+z}{2}\right)^{2} \mathrm{H}\left(\frac{m}{2}+\left\lfloor\frac{z+x}{2}\right\rfloor\right) \mathrm{H}\left(\frac{m}{2}+\left\lceil\frac{z+x}{2}\right\rceil\right)}{\mathrm{H}\left(\frac{m}{2}+\left\lfloor\frac{x+y+z}{2}\right\rfloor\right) \mathrm{H}\left(\frac{m}{2}+\left\lceil\frac{x+y+z}{2}\right\rceil\right) \mathrm{H}\left(\left\lceil\frac{x+y}{2}\right\rceil+1\right) \mathrm{H}\left(\frac{y+z}{2}\right) \mathrm{H}\left(\left\lfloor\frac{z+x}{2}\right\rfloor-1\right)} \\
& \times \frac{\mathrm{H}\left(m+\left\lfloor\frac{x+y+z}{2}\right\rfloor\right) \mathrm{H}\left(m+\left\lceil\frac{x+y+z}{2}\right\rceil\right)}{\mathrm{H}\left(m+\left\lfloor\frac{x+y}{2}\right\rfloor-1\right) \mathrm{H}\left(m+\frac{y+z}{2}\right) \mathrm{H}\left(m+\left\lceil\frac{z+x}{2}\right\rceil+1\right)} P_{2}(x, y, z, m), \quad\left(\mathrm{T}^{\prime}-2\right)
\end{aligned}
$$

where $P_{2}(x, y, z, m)$ is the polynomial defined in the Conjecture 2 .

We also need the following well-known formula of Cohn, Larsen, and Propp for tiling number of the dented semihexagon in the statements of our main theorems.

For a sequence $\mathbf{a}:=\left(a_{i}\right)_{i=1}^{m}$, we denote $o_{a}:=\sum_{i \text { odd }} a_{i}$ and $e_{a}:=\sum_{i \text { even }} a_{i}$. Let $S\left(a_{1}, a_{2}, \ldots, a_{m}\right)$ denote the upper half of a hexagon of side-lengths $e_{a}, o_{a}, o_{a}, e_{a}, o_{a}, o_{a}$ in which $k:=\left\lfloor\frac{m}{2}\right\rfloor$ triangles of side-lengths $a_{1}, a_{3}, a_{5}, \ldots, a_{2 k+1}$ removed from the base, such that the distance between the $i$-th and the $(i+1)$-th triangles is $a_{2 i}$ (see Figure 3 for an example). We call the region $S\left(a_{1}, a_{2}, \ldots, a_{m}\right)$ a dented semihexagon. Cohn, Larsen, and Propp [12] interpreted semi-strict Gelfand-Tsetlin patterns as lozenge tilings of the dented semihexagon $S\left(a_{1}, a_{2}, \ldots, a_{m}\right)$, and obtained the following tiling formula

$$
\begin{aligned}
s\left(a_{1}, a_{2}, \ldots, a_{2 l-1}\right) & =s\left(a_{1}, a_{2}, \ldots, a_{2 l}\right) \\
& =\frac{1}{\mathrm{H}\left(a_{1}+a_{3}+a_{5}+\ldots+a_{2 l-1}\right)} \frac{\prod_{\substack{1 \leqslant i<j \leqslant 2 l-1 \\
j-i \text { odd }}} \mathrm{H}\left(a_{i}+a_{i+1}+\ldots+a_{j}\right)}{\prod_{\substack{1 \leqslant i<j \leqslant 2 l-1 \\
j-i \text { even }}} \mathrm{H}\left(a_{i}+a_{i+1}+\ldots+a_{j}\right)},
\end{aligned}
$$

where $s\left(a_{1}, a_{2}, \ldots, a_{m}\right)$ denotes the number of tilings of $S\left(a_{1}, a_{2}, \ldots, a_{m}\right)$. 


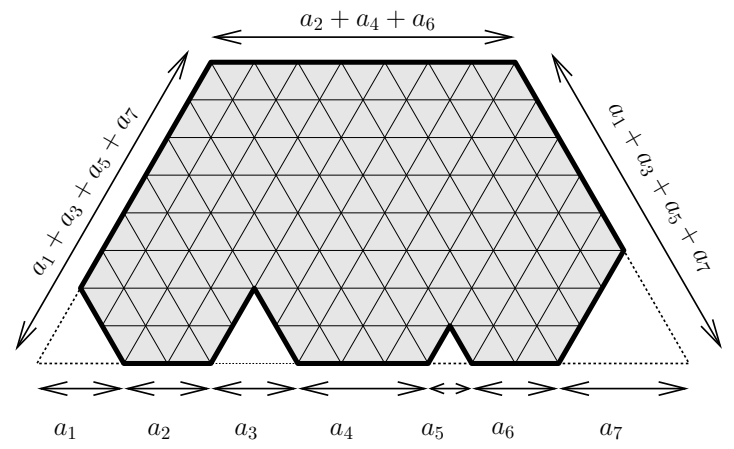

Figure 3: The dented semihexagon $S(2,2,2,3,1,2,4)$.

\subsection{The $E^{(i)}$-type regions}

We will define our region similarly to the cored hexagons. In particular, we start from an auxiliary hexagon $H_{0}$, and push out its sides in a certain way to obtain a larger hexagon, called the base hexagon. Then we will remove three collinear ferns from the base hexagon in a particular way to obtain our regions.

Let us investigate first the case when the auxiliary hexagon $H_{0}$ has side-lengths $x, z, z, x, z, z$ with $x$ and $z$ are of the same parity. We assume that the middle fern is removed at 1 unit from the center of the auxiliary hexagon. In this case, the center of $H_{0}$ is a lattice point, and there are six different lattice points, which have distance 1 from the center (shown in Figure 4(a); the darker shaded nodes indicate the center of the auxiliary hexagon $H_{0}$, and the lighter shaded nodes represent the possible off-central positions for the leftmost of the middle fern). We note that, in the case of cored hexagons, we only need to consider the regions corresponding to one of these six positions, since regions corresponding to other positions can be obtained from the latter region by $60^{\circ}$ rotations. However, this is not true anymore for our hexagons with three ferns removed.

Assume that $x$ and $z$ are nonnegative integers with the same parity, that $\mathbf{a}=\left(a_{1}, a_{2}, \ldots, a_{m}\right), \mathbf{b}:=\left(b_{1}, b_{2}, \ldots, b_{n}\right)$, and $\mathbf{c}=\left(c_{1}, c_{2}, \ldots, c_{k}\right)$ are three (possibly empty) sequences of nonnegative integers, and that $y$ is an integer that may take negative values in certain situations. Three sequences $\mathbf{a}, \mathbf{b}$, and $\mathbf{c}$ determine the side-lengths of triangles in the left, the right, and the central ferns, respectively. Set

$$
\begin{aligned}
& a:=\sum_{i=1}^{m} a_{i}, \quad b:=\sum_{j=1}^{n} b_{j}, \quad c:=\sum_{t=1}^{k} c_{t} \\
& e_{a}:=\sum_{i \text { even }} a_{i}, \quad o_{a}:=\sum_{i \text { odd }} a_{i}, \\
& e_{b}:=\sum_{j \text { even }} b_{j}, \quad o_{b}:=\sum_{j \text { odd }} b_{j}, \\
& e_{c}:=\sum_{t \text { even }} c_{t}, \quad o_{c}:=\sum_{t \text { odd }} c_{t} .
\end{aligned}
$$




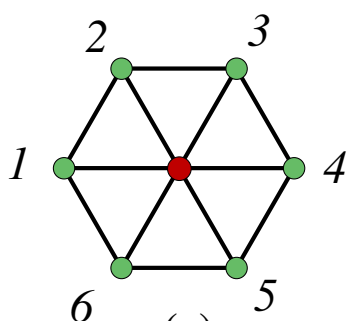

(a)

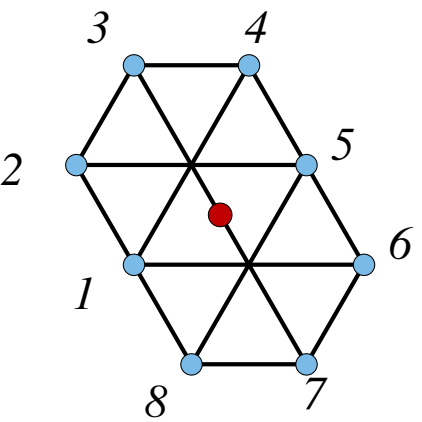

(c)

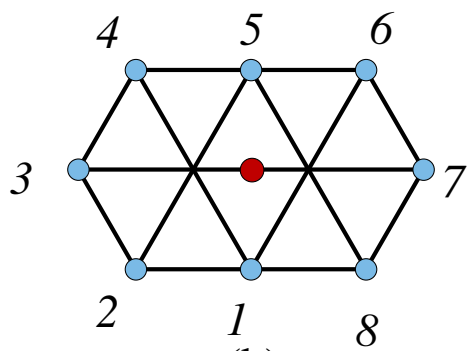

(b)

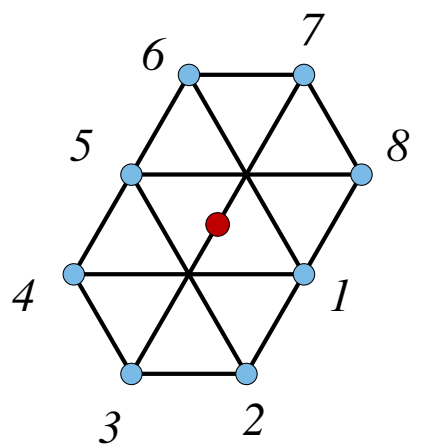

(d)

$[\mathrm{h}]$

Figure 4: (a) Six off-central positions for $E^{(i)}$ - and $\bar{E}^{(i)}$-type regions. (b) Eight off-central positions for $F^{(i)}$ - and $\bar{F}^{(i)}$-type regions. (c) Eight off-central positions for $G^{(i)_{-}}$and $\bar{G}^{(i)}$-type regions. (d) Eight off-central positions for $K^{(i)}-$ an $\bar{K}^{(i)}$-type regions.

We first define the regions corresponding to the off-central positions 1 and 4 in Figure 4(a). In this case, we assume further that $y \geqslant 0$ (the domain of the $y$-parameter changes in the other off-central cases). Let us start with the auxiliary hexagon $H_{0}$ of side-lengths $x, z, z, x, z, z$. We perform the following side-pushing process. We push outward all six sides of the hexagon (in clockwise order, starting from the north side) a distance of $e_{a}+o_{b}+o_{c}, y+\max (b-a, 0), b+c, b+c+y+\max (a-b, 0), o_{a}+e_{b}+e_{c}+y+\max (a-$ $b, 0), a, a+y+\max (b-a, 0)$ units, respectively. We get a larger hexagon $H$, called the base hexagon, which has side-lengths $x+o_{a}+e_{b}+e_{c}, 2 y+z+e_{a}+o_{b}+o_{c}+|a-b|$, $z+o_{a}+e_{b}+e_{c}, x+e_{a}+o_{b}+o_{c}, 2 y+z+o_{a}+e_{b}+e_{c}+|a-b|, z+e_{a}+o_{b}+o_{c}$. This is illustrated by Figure 5 (for $j=0$ ); the auxiliary hexagon is the shaded one with the dashed boundary, and the base hexagon is the one restricted by the bold contour.

For $i=1,4$, we denote by $E_{x, y, z}^{(i)}(\mathbf{a} ; \mathbf{c} ; \mathbf{b})$ the region obtained from the base hexagon $H$ by removing three ferns as follows. The middle fern consists of equilateral triangles of sidelengths $c_{1}, c_{2}, \ldots, c_{k}$ as they appear from left to right. The first triangle (of side-length $c_{1}$ ) is up-pointing, and the next triangles in the fern are oriented in alternating orientations. We remove the middle fern, such that its 'root' (its leftmost point) is at the off-central position $i$ as shown in Figure 4(a), for $i=1,4$. The left fern consists of triangles of sidelengths $a_{1}, a_{2}, \ldots, a_{m}$ running from left to right and starts by a down-pointing triangle, 


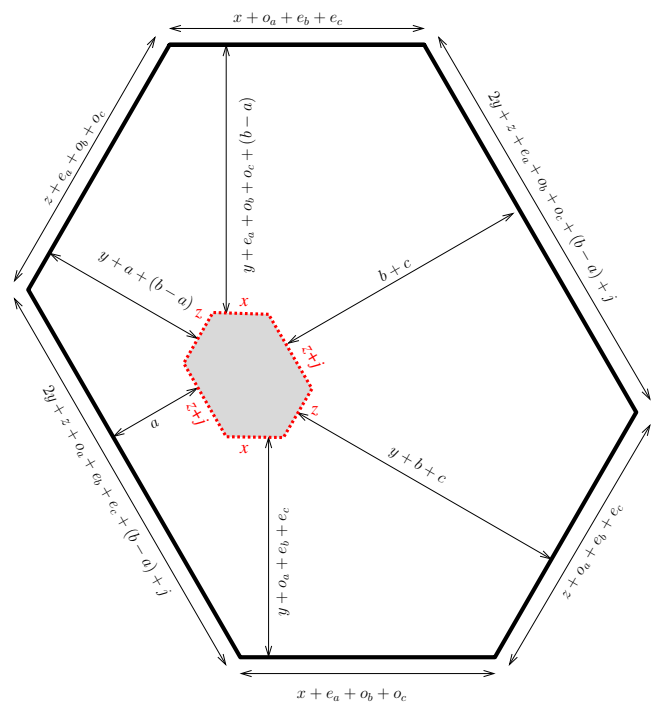

(a)

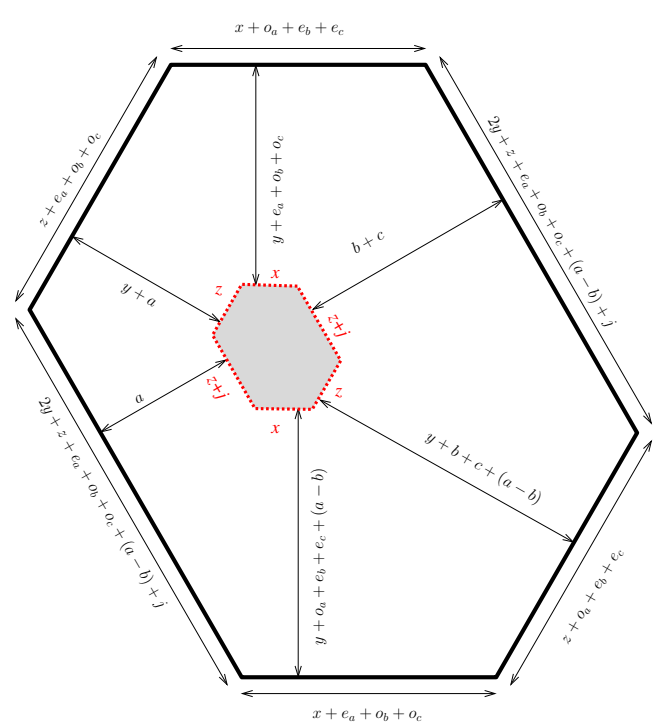

(b)

Figure 5: The edge-pushing procedure used in the definitions of the $E^{(i)_{-}}, F^{(i)_{-}}, G^{(i)_{-}}$, $K^{(i)}$-type regions: (a) the case $a \leqslant b$, (b) the case $a \geqslant b$.

while the right fern consists of triangles of side-lengths $b_{1}, b_{2}, \ldots, b_{n}$ running from right to left and starts by an up-pointing triangle. These latter two ferns are removed such that they are at the same level as the middle fern, that the left fern is touching the southwest side of the hexagon, and that the right fern is touching the northeast side of the hexagon. See Figure 6(a) for an example, in which the removed triangles are indicated by the black ones $^{2}$ ).

For $i=2,3,5,6$, unlike the case $i=1,4$ above, we start with an auxiliary hexagon of side-lengths $x, z+2, z, x, z+2, z$, we still perform the same side-pushing procedure to get the base hexagon of side-lengths $x+o_{a}+e_{b}+e_{c}, 2 y+z+e_{a}+o_{b}+o_{c}+|a-b|+2$, $z+o_{a}+e_{b}+e_{c}, x+e_{a}+o_{b}+o_{c}, 2 y+z+o_{a}+e_{b}+e_{c}+|a-b|+2, z+e_{a}+o_{b}+o_{c}$. If $i=2,3$, we allow $y$ to take integer values greater or equal to $\max (a-b,-2)$. In particular, $y \geqslant 0$ if $a \leqslant b$, however, $y$ may be negative when $a>b$. Our side-pushing process still works well in the same way as the definition of the $E^{(1)}$-type regions. We note that, even though $y$ may be negative, all the pushing distances are still nonnegative. Next, the three ferns are removed similarly, such that the root of the middle fern is at the off-central position $i$ in Figure 4(a), for $i=2,3$. If $i=5,6$, we also assume that $y \geqslant \max (b-a,-2)$, and the $E^{(5)}$ - and $E^{(6)}$-type regions are defined similarly. By the symmetry, it is enough to enumerate only three of the above regions, namely the $E^{(1)}-, E^{(2)}-$ and $E^{(6)}$-type regions (see Figure 6 for examples, as the remaining regions can be obtained from these three regions by $180^{\circ}$-rotations.

\footnotetext{
${ }^{2}$ In this section, we ignore four shaded triangles of labels $u, v, w, s$ in the illustrative figures; these triangles will be used later in Section 3.
} 
Remark 3. In the definition of the $E^{(1)}$-type regions, if we remove the three ferns such that the root of the middle one is at the center of the auxiliary hexagon $H_{0}$, then we obtain the region $R_{x, y, z}^{\odot}(\mathbf{a} ; \mathbf{c} ; \mathbf{b})$ in Theorem 2.2 of [31]. In some sense, the $E^{(i)}$-type regions can be viewed as off-central counterparts of the $R^{\odot}$-type regions investigated in [31].

We note that the positions of the two side ferns are determined uniquely by the level of the middle fern. Thus, from now on, when defining the three removed ferns, we only need to care about the position of the middle fern.

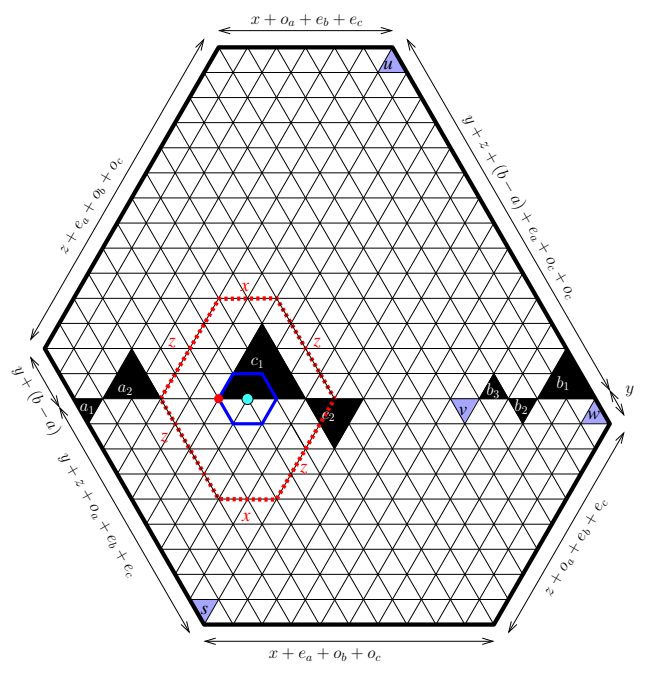

(a)

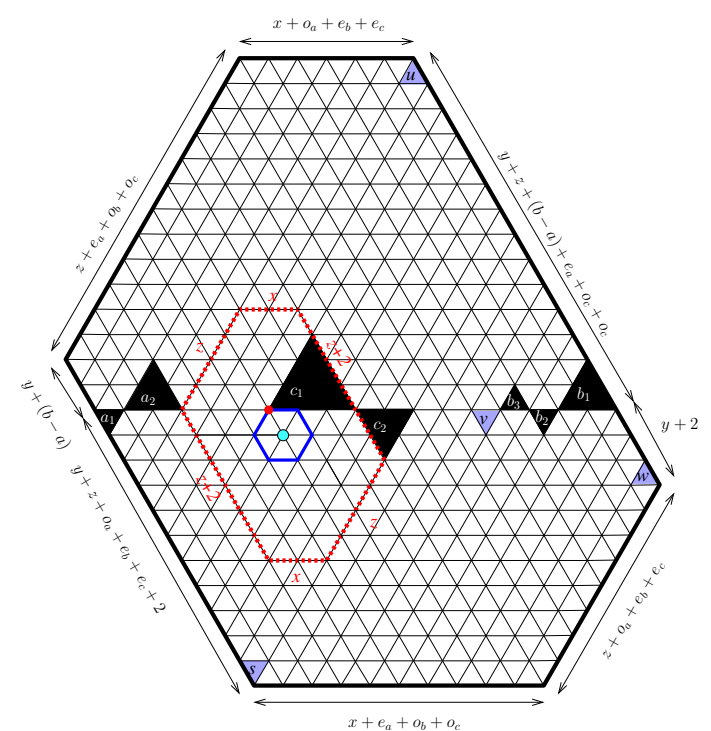

(b)

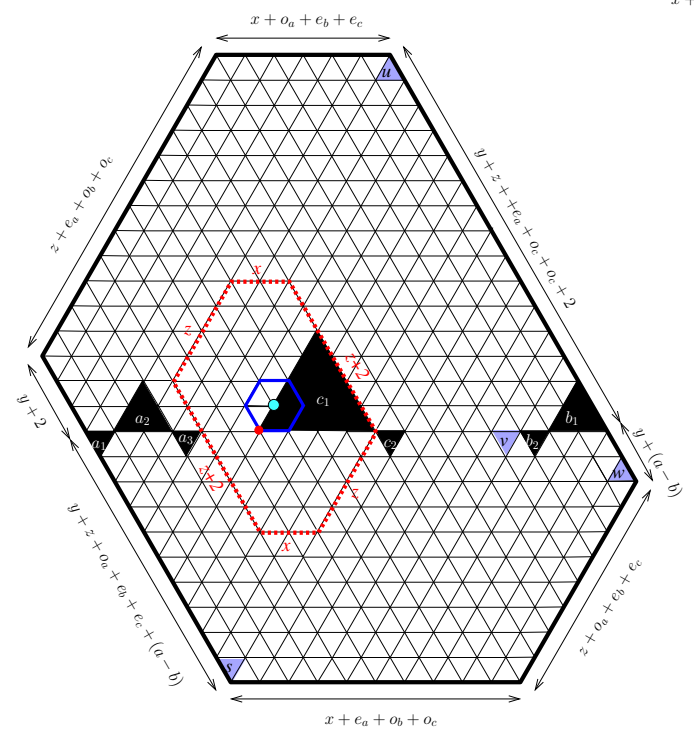

(c)

Figure 6: (a) The region $E_{2,1,4}^{(1)}(1,2 ; 3,2 ; 2,1,1)$. (b) The region $E_{2,1,4}^{(2)}(1,2 ; 3,2 ; 2,1,1)$. (c) The region $E_{2,1,4}^{(6)}(1,2,1 ; 4,1 ; 2,1)$

Before stating our enumerations, we note that one can always assume that each of our ferns has an even number of triangles. Indeed, if a fern has an odd number of triangles, 
we can regard that it contains an even number of triangles by adding a triangle of sidelength 0 to its end. For the sake of simplicity, we assume this in the statements of all our theorems throughout this paper.

Theorem 4. Assume that $\boldsymbol{a}=\left(a_{1}, a_{2}, \ldots, a_{m}\right), \boldsymbol{b}=\left(b_{1}, b_{2}, \ldots, b_{n}\right), \boldsymbol{c}=\left(c_{1}, c_{2}, \ldots, c_{k}\right)$ are three sequences of an even number of nonnegative integers ( $m, n, k$ are all even) and that $x, y, z$ are three nonnegative integers, such that $x$ and $z$ have the same parity. Then

$$
\begin{aligned}
\mathrm{M} & \left(E_{x, y, z}^{(1)}(\boldsymbol{a} ; \boldsymbol{c} ; \boldsymbol{b})\right)=\Phi_{x, 2 y+z+2 \max (a, b), z}(c) \\
& \times s\left(y+b-\min (a, b), a_{1}, \ldots, a_{m}, \frac{x+z}{2}-1, c_{1}, \ldots, c_{k}+\frac{x+z}{2}+1+b_{n}, b_{n-1}, \ldots, b_{1}\right) \\
& \times s\left(a_{1}, \ldots, a_{m-1}, a_{m}+\frac{x+z}{2}-1+c_{1}, c_{2},\right. \\
& \left.\ldots, c_{k}, \frac{x+z}{2}+1, b_{n}, \ldots, b_{1}, y+a-\min (a, b)\right) \\
& \times \frac{\mathrm{H}\left(c+\frac{x+z}{2}-1\right)}{\mathrm{H}(c) \mathrm{H}\left(\frac{x+z}{2}-1\right)} \frac{\mathrm{H}\left(\max (a, b)+y+\frac{x+z}{2}-1\right)}{\mathrm{H}\left(\max (a, b)+c+y+\frac{x+z}{2}-1\right)} \\
& \times \frac{\mathrm{H}(\max (a, b)+y+z) \mathrm{H}(\max (a, b)+c+y+z)}{\mathrm{H}\left(\max (a, b)-o_{a}+o_{b}+o_{c}+y+z\right) \mathrm{H}\left(\max (a, b)+o_{a}-o_{b}+e_{c}+y+z\right)} \\
& \times \frac{\mathrm{H}\left(\max (a, b)-o_{a}+o_{b}+o_{c}+y\right) \mathrm{H}\left(\max (a, b)+o_{a}-o_{b}+e_{c}+y\right)}{\mathrm{H}(\max (a, b)+y)^{2}}
\end{aligned}
$$

where $\Phi_{x, y, z}(m)$ is defined as in (T-1) of Conjecture 1 and where $a, b, c, o_{a}, o_{b}, o_{c}, e_{a}, e_{b}, e_{c}$ are defined as in (7).

Theorem 5. Assume that $\boldsymbol{a}=\left(a_{1}, a_{2}, \ldots, a_{m}\right), \boldsymbol{b}=\left(b_{1}, b_{2}, \ldots, b_{n}\right), \boldsymbol{c}=\left(c_{1}, c_{2}, \ldots, c_{k}\right)$ are three sequences of nonnegative integers ( $m, n, k$ are all even) and that $x, y, z$ are three integers, such that $x, z \geqslant 0, y \geqslant \max (a-b,-2)$, and $x$ and $z$ have the same parity. Then

$$
\begin{aligned}
\mathrm{M} & \left(E_{x, y, z}^{(2)}(\boldsymbol{a} ; \boldsymbol{c} ; \boldsymbol{b})\right)=\Phi_{2 y+z+2 \max (a, b)+2, x, z}(c) \\
& \times s\left(y+b-\min (a, b), a_{1}, \ldots, a_{m}, \frac{x+z}{2}, c_{1}, \ldots, c_{k}+\frac{x+z}{2}+b_{n}, b_{n-1}, \ldots, b_{1}\right) \\
& \times s\left(a_{1}, \ldots, a_{m-1}, a_{m}+\frac{x+z}{2}+c_{1}, \ldots, c_{k}, \frac{x+z}{2}, b_{n}, \ldots, b_{1}, y+a-\min (a, b)+2\right) \\
& \times \frac{\mathrm{H}\left(c+\frac{x+z}{2}\right)}{\mathrm{H}(c) \mathrm{H}\left(\frac{x+z}{2}\right)} \frac{\mathrm{H}\left(\max (a, b)+y+\frac{x+z}{2}\right)}{\mathrm{H}\left(\max (a, b)+c+y+\frac{x+z}{2}\right)} \\
& \times \frac{\mathrm{H}(\max (a, b)+y+z+2) \mathrm{H}(\max (a, b)+c+y+z)}{\mathrm{H}\left(\max (a, b)-o_{a}+o_{b}+o_{c}+y+z\right) \mathrm{H}\left(\max (a, b)+o_{a}-o_{b}+e_{c}+y+z+2\right)} \\
& \times \frac{\mathrm{H}\left(\max (a, b)-o_{a}+o_{b}+o_{c}+y\right) \mathrm{H}\left(\max (a, b)+o_{a}-o_{b}+e_{c}+y+2\right)}{\mathrm{H}(\max (a, b)+y) \mathrm{H}(\max (a, b)+y+2)} .
\end{aligned}
$$


Theorem 6. Assume that $\boldsymbol{a}=\left(a_{1}, a_{2}, \ldots, a_{m}\right), \boldsymbol{b}=\left(b_{1}, b_{2}, \ldots, b_{n}\right), \boldsymbol{c}=\left(c_{1}, c_{2}, \ldots, c_{k}\right)$ are three sequences of nonnegative integers (for $m, n, k$ are even) and that $x, y, z$ are three integers, such that $x, z \geqslant 0, y \geqslant \max (b-a,-2)$, and $x$ and $z$ have the same parity. Then

$$
\begin{aligned}
& \mathrm{M}\left(E_{x, y, z}^{(6)}(\boldsymbol{a} ; \boldsymbol{c} ; \boldsymbol{b})\right)=\Phi_{z, 2 y+z+2 \max (a, b)+2, x}(c) \\
& \quad \times s\left(y+b-\min (a, b)+2, a_{1}, \ldots, a_{m}, \frac{x+z}{2}-1, c_{1},\right. \\
& \left.\quad \ldots, c_{k}+\frac{x+z}{2}+1+b_{n}, b_{n-1}, \ldots, b_{1}\right) \\
& \quad \times s\left(a_{1}, \ldots, a_{m-1}, a_{m}+\frac{x+z}{2}-1+c_{1}, c_{2}\right. \\
& \left.\quad \ldots, c_{k}, \frac{x+z}{2}+1, b_{n}, \ldots, b_{1}, y+a-\min (a, b)\right) \\
& \quad \times \frac{\mathrm{H}\left(c+\frac{x+z}{2}-1\right)}{\mathrm{H}(c) \mathrm{H}\left(\frac{x+z}{2}-1\right) \frac{\mathrm{H}\left(\max (a, b)+y+\frac{x+z}{2}+1\right)}{\mathrm{H}\left(\max (a, b)+c+y+\frac{x+z}{2}+1\right)}} \\
& \quad \times \frac{\mathrm{H}(\max (a, b)+y+z) \mathrm{H}(\max (a, b)+c+y+z+2)}{\mathrm{H}\left(\max (a, b)-o_{a}+o_{b}+o_{c}+y+z+2\right) \mathrm{H}\left(\max (a, b)+o_{a}-o_{b}+e_{c}+y+z\right)}
\end{aligned}
$$

One could see that the factor involving function $\Phi$ in formula (9) (resp., formula (10)) is obtained from that in the formula (8) by interchanging the $x$ and $y$ (resp., $x$ and $z$ ).

Next, we show that Theorem 4 implies Ciucu-Eisenkölbl-Krattenthaler-Zare's Conjecture 1 .

Proof of Conjecture 1 by using Theorem 4. Applying formula (8) in Theorem 4 to the region

$E_{x, \frac{y-z}{2}, z}^{(1)}(\varnothing ; m ; \varnothing)$, we prove the conjecture for the case when $y \geqslant z$ (all factors in (8), except for the first one, cancel out). The remaining case, when $y<z$, is obtained by applying Theorem 4 to a horizontal reflection of the region $E_{x, \frac{z-y}{2}, y}^{(1)}(\varnothing ; 0, m ; \varnothing)$. Here the middle fern consists of a 0 -triangle and an $m$-triangle.

Remark 7 (Geometric Interpretation). One can rewrite the equation (8) in Theorem 4 as

$$
\begin{aligned}
& \mathrm{M}\left(E_{x, y, z}^{(1)}(\mathbf{a} ; \mathbf{c} ; \mathbf{b})\right)=\mathrm{M}\left(C_{x, 2 y+z+2 \max (a, b), z}^{(1)}(c)\right) \\
& \times s\left(y+b-\min (a, b), a_{1}, \ldots, a_{m}, \frac{x+z}{2}-1, c_{1}, \ldots, c_{k}+\frac{x+z}{2}+1+b_{n}, b_{n-1}, \ldots, b_{1}\right) \\
& \times s\left(a_{1}, \ldots, a_{m-1}, a_{m}+\frac{x+z}{2}-1+c_{1}, c_{2},\right. \\
& \left.\ldots, c_{k}, \frac{x+z}{2}+1, b_{n}, \ldots, b_{1}, y+a-\min (a, b)\right)
\end{aligned}
$$




$$
\frac{\operatorname{PP}\left(y+\max (a, b)-o_{a}+o_{b}+o_{c}, y+\max (a, b)+o_{a}-o_{b}+e_{c}, z\right)}{\operatorname{PP}\left(\frac{x+z}{2}-1, c, y+\max (a, b)\right) \operatorname{PP}(y+\max (a, b)+c, y+\max (a, b), z)},
$$

where $C_{x, y, z}^{(1)}(m)$ is the off-central cored hexagon in Conjecture 1 and where

$$
\operatorname{PP}(a, b, c):=\frac{\mathrm{H}(a) \mathrm{H}(b) \mathrm{H}(c) \mathrm{H}(a+b+c)}{\mathrm{H}(a+b) \mathrm{H}(b+c) \mathrm{H}(c+a)}
$$

is the tiling number of the centrally symmetric hexagon of side-lengths $a, b, c, a, b, c$ (it is also the number of plane partitions fitting in an $a \times b \times c$-box by MacMahon's celebrated theorem [35]). We have a similar interpretation for other theorems in this section. This would be interesting to have a combinatorial proof for this.

\subsection{The $F^{(i)}$-type regions}

We now consider a similar situation to the case of $E^{(i)}$-type regions; however, $x$ and $z$ now have opposite parities. Let $x, z$ be two nonnegative integers, $y$ an integer that may be negative, and $\mathbf{a}, \mathbf{b}, \mathbf{c}$ three sequences of nonnegative integers that record the side-lengths of triangles in our ferns as usual. In this case, the center of our auxiliary hexagon $H_{0}$ is the middle point of a horizontal unit lattice segment. There are a total of eight offcentral positions to remove the middle fern corresponding to the positions $1,2,3, \ldots, 8$ in Figure 4(b). For $i=1,2,4,5,6,8$, we start with the auxiliary hexagon $H_{0}$ of side-lengths $x, z+2, z, x, z+2, z$, while $i=3,7$, we start with the auxiliary hexagon of side-lengths $x, z, z, x, z, z$. We assume in addition that $y \geqslant 0$ if $i=3,7, y \geqslant \max (a-b,-2)$ if $i=4,5,6$, and $y \geqslant \max (b-a,-2)$ if $i=1,2,8$. Next, we apply the side-pushing procedure in the definition of the $E^{(i)}$-type regions to obtain the base hexagon $H$. We also remove similarly three collinear ferns from $H$ at the same level, such that the root of the middle fern is at the off-central position $i$, as in Figure 4(b). (The two side ferns are also defined uniquely such that the left fern is touching the southwest side and the right fern is touching the northeast side of the base hexagon.) Denote by $F_{x, y, z}^{(i)}(\mathbf{a} ; \mathbf{c} ; \mathbf{b})$ the resulting region, $i=1,2, \ldots, 8$. However, by symmetry, we only need to enumerate four of them, namely the ones corresponding to the first, the second, the third, and the fourth off-central positions (see Figure 7 for examples of these regions).

Remark 8. In the definition of the $F^{(3)}$-type regions, if we remove the three ferns such that the root of the middle one is $1 / 2$-unit to the left of the center of the auxiliary hexagon $H_{0}$, then we obtain exactly the region $R_{x, y, z}^{\leftarrow}(\mathbf{a} ; \mathbf{c} ; \mathbf{b})$ in Theorem 2.3 of [31]. In some sense, the $F^{(i)}$-type regions can be viewed as off-central counterparts of the $R^{\leftarrow}$-type regions.

Theorem 9. Assume that $\boldsymbol{a}=\left(a_{1}, a_{2}, \ldots, a_{m}\right), \boldsymbol{b}=\left(b_{1}, b_{2}, \ldots, b_{n}\right), \boldsymbol{c}=\left(c_{1}, c_{2}, \ldots, c_{k}\right)$ are three sequences of nonnegative integers ( $m, n, k$ are even) and that $x, y, z$ are three integers, such that $x \geqslant 0, y \geqslant \max (b-a,-2), z \geqslant 0$, and $x$ has parity opposite to $z$. Then

$$
\mathrm{M}\left(F_{x, y, z}^{(1)}(\boldsymbol{a} ; \boldsymbol{c} ; \boldsymbol{b})\right)=\Theta_{x, 2 y+z+2 \max (a, b)+2, z}(c)
$$




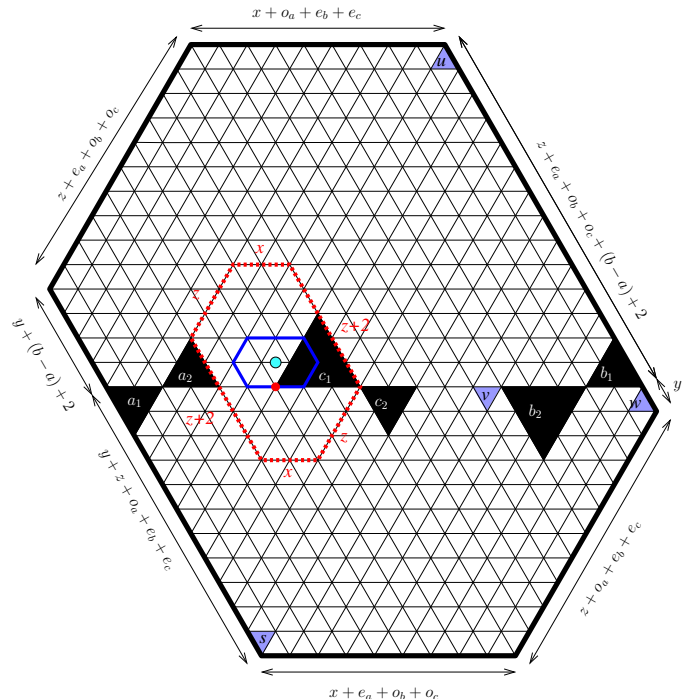

(a)

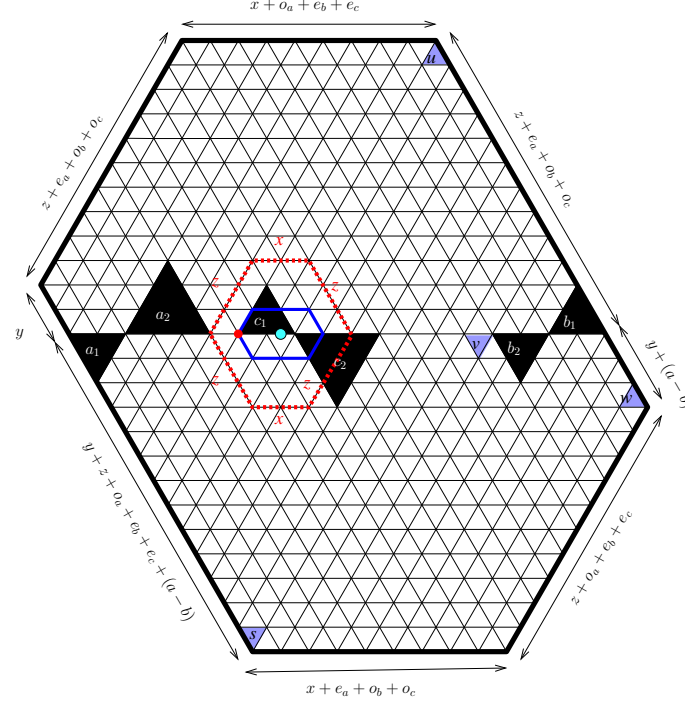

(c)

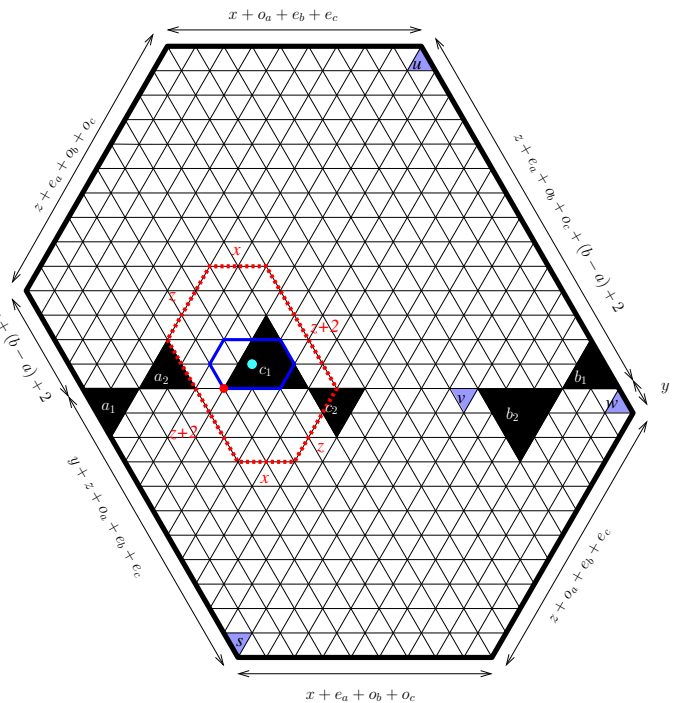

(b)

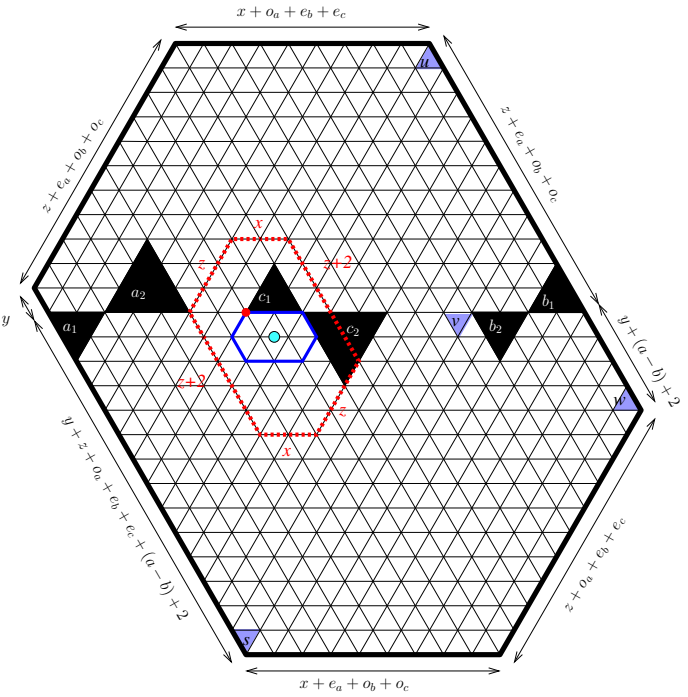

(d)

Figure 7: The four $F^{(i)}$-type regions: (a) $F_{2,1,3}^{(1)}(2,2 ; 3,2 ; 2,3)$, (b) $F_{2,1,3}^{(2)}(2,2 ; 3,2 ; 2,3)$, (c) $F_{2,2,3}^{(3)}(2,3 ; 2,3 ; 2,2)$, and (d) $F_{2,1,3}^{(4)}(2,3 ; 2,3 ; 2,2)$.

$$
\begin{aligned}
& \times s\left(y+b-\min (a, b)+2, a_{1}, \ldots, a_{m},\left\lfloor\frac{x+z}{2}\right\rfloor, c_{1},\right. \\
& \left.\ldots, c_{k}+\left\lceil\frac{x+z}{2}\right\rceil+b_{n}, b_{n-1}, \ldots, b_{1}\right) \\
& \times s\left(a_{1}, \ldots, a_{m-1}, a_{m}+\left\lfloor\frac{x+z}{2}\right\rfloor+c_{1}, \ldots, c_{k},\left\lceil\frac{x+z}{2}\right\rceil, b_{n}, \ldots, b_{1}, y+a-\min (a, b)\right)
\end{aligned}
$$




$$
\begin{aligned}
& \times \frac{\mathrm{H}\left(c+\left\lfloor\frac{x+z}{2}\right\rfloor\right)}{\mathrm{H}(c) \mathrm{H}\left(\left\lfloor\frac{x+z}{2}\right\rfloor\right)} \frac{\mathrm{H}\left(\max (a, b)+y+\left\lceil\frac{x+z}{2}\right\rceil+1\right)}{\mathrm{H}\left(\max (a, b)+c+y+\left\lceil\frac{x+z}{2}\right\rceil+1\right)} \\
& \times \frac{\mathrm{H}(\max (a, b)+y+z) \mathrm{H}(\max (a, b)+c+y+z+2)}{\mathrm{H}\left(\max (a, b)-o_{a}+o_{b}+o_{c}+y+z+2\right) \mathrm{H}\left(\max (a, b)+o_{a}-o_{b}+e_{c}+y+z\right)} \\
& \times \frac{\left.\mathrm{H} \max (a, b)-o_{a}+o_{b}+o_{c}+y+2\right) \mathrm{H}\left(\max (a, b)+o_{a}-o_{b}+e_{c}+y\right)}{\mathrm{H}(\max (a, b)+y) \mathrm{H}(\max (a, b)+y+2)},
\end{aligned}
$$

where $\Theta_{x, y, z}(m)$ is given by (T-3).

Unlike the tiling formulas for the $E^{(i)}$-type regions above, which have the non-linear term $P_{1}(x, y, z, m)$, this formula is a 'simple' product formula, in the sense that the largest factor is linear in the parameters of the region. Moreover, the corresponding cored hexagons of these regions have not appeared in the previous work of Ciucu-EisenkölblKrattenthaler-Zare [7].

Theorem 9 gives an exact enumeration for a new type of cored hexagons with the triangular hole down-pointing.

Corollary 10. Let $x, y, z, m$ be nonnegative integers. The number of tilings of the cored hexagon, in which the down-pointing triangular hole has side-length $m$ and its leftmost at position 1 as in Figure 4(b), is equal to $\Theta_{x, y, z}^{\prime}(m)$.

Proof. The case when $y \geqslant z$ follows Theorem 9 for the region $F_{x, \frac{y-z}{2}, z}^{(1)}(\varnothing ; 0, m ; \varnothing)$. The case when $y<z$ is obtained by applying Theorem 9 to a vertical reflection of the region $F_{x, \frac{z-y}{2}, z}^{(1)}(\varnothing ; 0, m ; \varnothing)$.

Theorem 11. Assume that $\boldsymbol{a}=\left(a_{1}, a_{2}, \ldots, a_{m}\right), \boldsymbol{b}=\left(b_{1}, b_{2}, \ldots, b_{n}\right), \boldsymbol{c}=\left(c_{1}, c_{2}, \ldots, c_{k}\right)$ are three sequences of nonnegative integers ( $m, n, k$ are even) and that $x, y, z$ are three integers, such that $x \geqslant 0, y \geqslant \max (b-a,-2), z \geqslant 0$, and $x$ and $z$ have opposite parities. We have

$$
\begin{aligned}
& \mathrm{M}\left(F_{x, y, z}^{(2)}(\boldsymbol{a} ; \boldsymbol{c} ; \boldsymbol{b})\right)=\Lambda_{x, 2 y+z+2 \max (a, b)+2, z}(c) \\
& \quad \times s\left(y+b-\min (a, b)+2, a_{1}, \ldots, a_{m},\left\lfloor\frac{x+z}{2}\right\rfloor-1, c_{1},\right. \\
& \left.\ldots, c_{k}+\left\lceil\frac{x+z}{2}\right\rfloor+b_{n}+1, b_{n-1}, \ldots, b_{1}\right) \\
& \quad \times s\left(a_{1}, \ldots, a_{m-1}, a_{m}+\left\lfloor\frac{x+z}{2}\right\rfloor-1+c_{1}, c_{2}\right. \\
& \left.\quad \ldots, c_{k},\left\lceil\frac{x+z}{2}\right\rfloor+1, b_{n}, \ldots, b_{1}, y+a-\min (a, b)\right) \\
& \quad \times \frac{\mathrm{H}\left(c+\left\lfloor\frac{x+z}{2}\right\rfloor-1\right)}{\mathrm{H}(c) \mathrm{H}\left(\left\lfloor\frac{x+z}{2}\right\rfloor-1\right)} \frac{\mathrm{H}\left(\max (a, b)+y+\left\lfloor\frac{x+z}{2}\right\rfloor+1\right)}{\mathrm{H}\left(\max (a, b)+c+y+\left\lfloor\frac{x+z}{2}\right\rfloor+1\right)} \\
& \quad \times \frac{\mathrm{max}(a, b)+y+z) \mathrm{H}(\max (a, b)+c+y+z+2)}{\mathrm{H}\left(\max (a, b)-o_{a}+o_{b}+o_{c}+y+z+2\right) \mathrm{H}\left(\max (a, b)+o_{a}-o_{b}+e_{c}+y+z\right)}
\end{aligned}
$$




$$
\times \frac{\mathrm{H}\left(\max (a, b)-o_{a}+o_{b}+o_{c}+y+2\right) \mathrm{H}\left(\max (a, b)+o_{a}-o_{b}+e_{c}+y\right)}{\mathrm{H}(\max (a, b)+y) \mathrm{H}(\max (a, b)+y+2)} .
$$

where $\Lambda_{x, y, z}(m)$ is defined as in $(T-4)$.

Theorem 12. Assume that $\boldsymbol{a}=\left(a_{1}, a_{2}, \ldots, a_{m}\right), \boldsymbol{b}=\left(b_{1}, b_{2}, \ldots, b_{n}\right), \boldsymbol{c}=\left(c_{1}, c_{2}, \ldots, c_{k}\right)$ are three sequences of nonnegative integers ( $m, n, k$ are all even) and that $x, y, z$ are three nonnegative integers, such that $x$ has parity opposite to $z$. Then

$$
\begin{aligned}
& \mathrm{M}\left(F_{x, y, z}^{(3)}(\boldsymbol{a} ; \boldsymbol{c} ; \boldsymbol{b})\right)=\Psi_{x, 2 y+z+2 \max (a, b), z}(c) \\
& \quad \times s\left(y+b-\min (a, b), a_{1}, \ldots, a_{m},\left\lfloor\frac{x+z}{2}\right\rfloor-1, c_{1},\right. \\
& \left.\quad \ldots, c_{k}+\left\lceil\frac{x+z}{2}\right\rfloor+b_{n}+1, b_{n-1}, \ldots, b_{1}\right) \\
& \quad \times s\left(a_{1}, \ldots, a_{m-1}, a_{m}+\left\lfloor\frac{x+z}{2}\right\rfloor-1+c_{1}, c_{2},\right. \\
& \left.\quad \ldots, c_{k},\left\lceil\frac{x+z}{2}\right\rceil+1, b_{n}, \ldots, b_{1}, y+a-\min (a, b)\right) \\
& \quad \times \frac{\mathrm{H}\left(c+\left\lfloor\frac{x+z}{2}\right\rfloor-1\right)}{\mathrm{H}(c) \mathrm{H}\left(\left\lfloor\frac{x+z}{2}\right\rfloor-1\right)} \frac{\mathrm{H}\left(\max (a, b)+y+\left\lfloor\frac{x+z}{2}\right\rfloor-1\right)}{\mathrm{H}\left(\max (a, b)+c+y+\left\lfloor\frac{x+z}{2}\right\rfloor-1\right)} \\
& \quad \times \frac{\mathrm{H}(\max (a, b)+y+z) \mathrm{H}(\max (a, b)+c+y+z)}{\mathrm{H}\left(\max (a, b)-o_{a}+o_{b}+o_{c}+y+z\right) \mathrm{H}\left(\max (a, b)+o_{a}-o_{b}+e_{c}+y+z\right)} \\
& \quad \times \frac{\mathrm{H}\left(\max (a, b)-o_{a}+o_{b}+o_{c}+y\right) \mathrm{H}\left(\max (a, b)+o_{a}-o_{b}+e_{c}+y\right)}{\mathrm{H}(\max (a, b)+y)^{2}},
\end{aligned}
$$

where $\Psi_{x, y, z}(m)$ is given by the formula (T-2) in Conjecture 2.

Next, we show that Theorem 12 has Conjecture 2 as a special case.

Proof of Conjecture 2 by using Theorem 12. Applying Theorem 12 to the region $F_{x, \frac{y-z}{2}, z}^{(3)}(\varnothing ; m ; \varnothing)$, we verify the conjecture for $y \geqslant z$ (all factors in (12), except for the first one, cancel out). The case $y<z$ is obtained by applying the theorem to a horizontal reflection of the region $F_{x, \frac{z-y}{2}, z}^{(3)}(\varnothing ; 0, m ; \varnothing)$.

We have an exact enumeration of a new type of cored hexagons whose triangular hole is down-pointing:

Corollary 13. Let $x, y, z, m$ be nonnegative integers. The number of tilings of the cored hexagon, in which the down-pointing triangular hole has its leftmost vertex at the third position as in Figure 4(b), equals $\Psi_{x, y, z}^{\prime}(m)$ in (T'-2).

We now have a tiling enumeration for the $F^{(4)}$-type regions: 
Theorem 14. Assume that $\boldsymbol{a}=\left(a_{1}, a_{2}, \ldots, a_{m}\right), \boldsymbol{b}=\left(b_{1}, b_{2}, \ldots, b_{n}\right), \boldsymbol{c}=\left(c_{1}, c_{2}, \ldots, c_{k}\right)$ are three sequences of nonnegative integers $(m, n, k$ are all even) and that $x, y, z$ are three integers, such that $x \geqslant 0, y \geqslant \max (a-b,-2), z \geqslant 0$, and $x$ has parity opposite to $z$. Then

$$
\begin{aligned}
& \mathrm{M}\left(F_{x, y, z}^{(4)}(\boldsymbol{a} ; \boldsymbol{c} ; \boldsymbol{b})\right)=\Lambda_{x, z, 2 y+z+2 \max (a, b)+2}^{\prime}(c) \\
& \quad \times s\left(y+b-\min (a, b), a_{1}, \ldots, a_{m},\left\lfloor\frac{x+z}{2}\right\rfloor, c_{1}, \ldots, c_{k}+\left\lceil\frac{x+z}{2}\right\rceil+b_{n}, b_{n-1}, \ldots, b_{1}\right) \\
& \quad \times s\left(a_{1}, \ldots, a_{m-1}, a_{m}+\left\lfloor\frac{x+z}{2}\right\rfloor+c_{1}, c_{2},\right. \\
& \left.\quad \ldots, c_{k},\left\lceil\frac{x+z}{2}\right\rfloor, b_{n}, \ldots, b_{1}, y+a-\min (a, b)+2\right) \\
& \quad \times \frac{\mathrm{H}\left(c+\left\lfloor\frac{x+z}{2}\right\rfloor\right)}{\mathrm{H}(c) \mathrm{H}\left(\left\lfloor\frac{x+z}{2}\right\rfloor\right)} \frac{\mathrm{H}\left(\max (a, b)+y+\left\lfloor\frac{x+z}{2}\right\rfloor\right)}{\mathrm{H}\left(\max (a, b)+c+y+\left\lfloor\frac{x+z}{2}\right\rfloor\right)} \\
& \quad \times \frac{\mathrm{H}(\max (a, b)+y+z+2) \mathrm{H}(\max (a, b)+c+y+z)}{\mathrm{H}\left(\max (a, b)-o_{a}+o_{b}+o_{c}+y+z\right) \mathrm{H}\left(\max (a, b)+o_{a}-o_{b}+e_{c}+y+z+2\right)} \\
& \quad \times \frac{\mathrm{H}\left(\max (a, b)-o_{a}+o_{b}+o_{c}+y\right) \mathrm{H}\left(\max (a, b)+o_{a}-o_{b}+e_{c}+y+2\right)}{\mathrm{H}(\max (a, b)+y) \mathrm{H}(\max (a, b)+y+2)}
\end{aligned}
$$

where $\Lambda_{x, y, z}^{\prime}(m)$ is defined as in $\left(T^{\prime}-4\right)$.

Corollary 15. Let $x, y, z, m$ be nonnegative integers. The number of tilings of the cored hexagon, in which the down-pointing triangular hole has its leftmost vertex at the second position as in Figure 4(b), equals $\Lambda_{x, y, z}^{\prime}(m)$ in $\left(T^{\prime}-4\right)$.

Proof. The case when $y \geqslant z$ follows Theorem 11 for the region $F_{x, \frac{y-z}{2}, z}^{(2)}(\varnothing ; 0, m ; \varnothing)$. The case when $y<z$ is obtained by applying Theorem 14 to a horizontal reflection of the region $F_{x, \frac{z-y}{2}, z}^{(4)}(\varnothing ; m ; \varnothing)$.

\subsection{The $G^{(i)}$-type regions}

We now consider the case when the center of the auxiliary hexagon is the middle point of a southeast-to-northwest unit lattice interval. There are also eight off-central positions labeled by $1,2, \ldots, 8$ around the center as shown in Figure $4(\mathrm{c})$. Let $x, z$ be two nonnegative integers with the same parity. In particular, for $i=1,2,5,6$, we consider the auxiliary hexagon $H_{0}$ of side-lengths $x, z+1, z, x, z+1, z$, while for $i=3,4,7,8$, the auxiliary hexagon has side-lengths $x, z+3, z, x, z+3, z$. The domain of the $y$-parameter can be defined in general as follows. If the position $i$ is $d$ units above the center of the auxiliary hexagon $(d=1 / 2$ or $3 / 2$ here), then $y \geqslant \max (a-b,-2 d)$; if $i$ is $d$ units below the center, then $y \geqslant \max (b-a,-2 d)$. Next, we apply the same side-pushing procedure as in the definition of the $E^{(i)}$-type regions to the new auxiliary hexagon to get the base 
hexagon $H$. We then remove the three ferns at the same level similarly, such that the middle fern has the root at the off-central position $i(i=1,2, \ldots, 8)$. Let us denote by $G_{x, y, z}^{(i)}(\mathbf{a} ; \mathbf{c} ; \mathbf{b})$ the newly defined region (see Figure 8 for examples). Again, by the symmetry, we only need to enumerate four of the eight regions above, namely $G^{(1)_{-}}, G^{(2)_{-}}$, $G^{(3)}{ }_{-}$, and $G^{(4)}$-type regions.

Remark 16. In the definition of the $G^{(1)}$-type regions, if we remove the three ferns such that the root of the middle one is $1 / 2$-unit to the northwest of the center of the auxiliary hexagon $H_{0}$, then we obtain the region $R_{x, y, z}^{\nwarrow}(\mathbf{a} ; \mathbf{c} ; \mathbf{b})$ in Theorem 2.4 of [31]. It means that the $G^{(i)}$-type regions can be viewed as off-central counterparts of the $R^{\nwarrow}$-type regions.

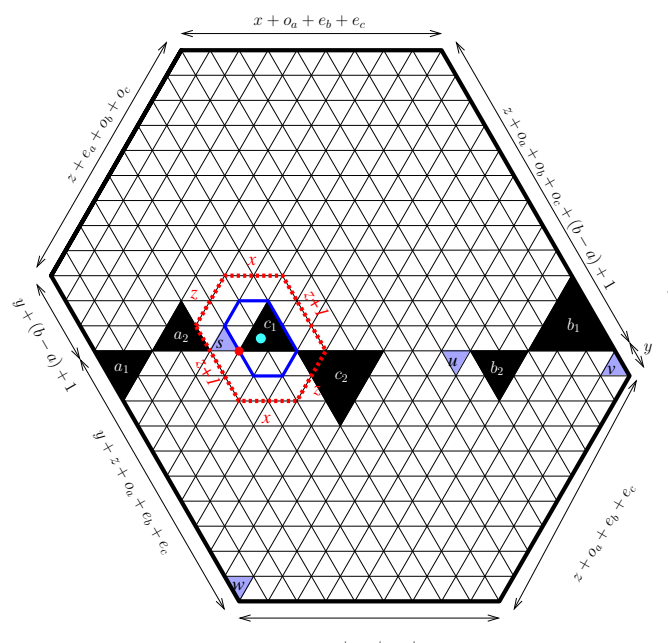

(a)

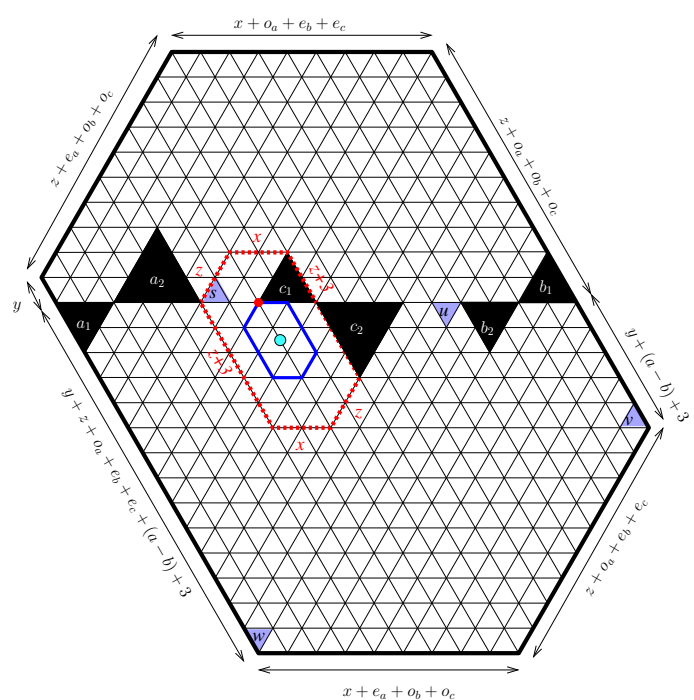

(c)

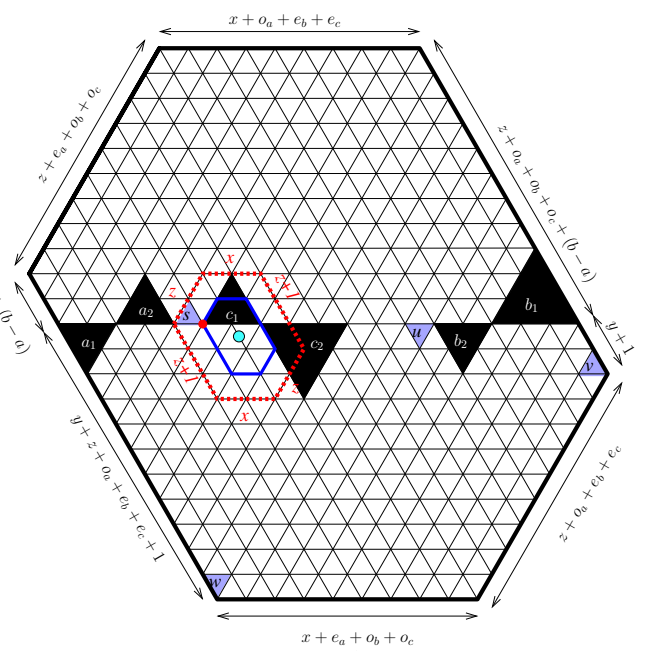

(b)

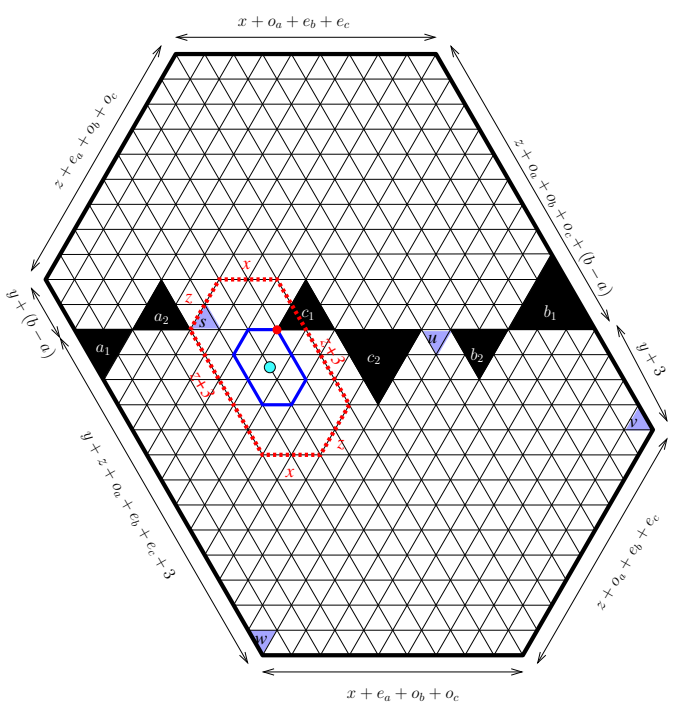

(d)

Figure 8: The four $G^{(i)}$-type regions: (a) $G_{2,1,2}^{(1)}(2,2 ; 2,3 ; 3,2)$, (b) $G_{2,1,2}^{(2)}(2,2 ; 2,3 ; 3,2)$, (c) $G_{2,1,2}^{(3)}(2,3 ; 2,3 ; 2,2)$, and (d) $G_{2,1,2}^{(4)}(2,2 ; 2,3 ; 3,2)$. 
Theorem 17. Assume that $\boldsymbol{a}=\left(a_{1}, a_{2}, \ldots, a_{m}\right), \boldsymbol{b}=\left(b_{1}, b_{2}, \ldots, b_{n}\right), \boldsymbol{c}=\left(c_{1}, c_{2}, \ldots, c_{k}\right)$ are three sequences of nonnegative integers ( $m, n, k$ are all even) and that $x, y, z$ are three integers, such that $x \geqslant 0, y \geqslant \max (b-a,-1), z \geqslant 0$, and $x$ and $z$ have the same parity. Then

$$
\begin{aligned}
& \mathrm{M}\left(G_{x, y, z}^{(1)}(\boldsymbol{a} ; \boldsymbol{c} ; \boldsymbol{b})\right)=\Theta_{2 y+z+2 \max (a, b)+1, z, x}^{\prime}(c) \\
& \quad \times s\left(y+b-\min (a, b)+1, a_{1}, \ldots, a_{m}, \frac{x+z}{2}-1, c_{1},\right. \\
& \left.\quad \ldots, c_{k}+\frac{x+z}{2}+1+b_{n}, b_{n-1}, \ldots, b_{1}\right) \\
& \quad \times s\left(a_{1}, \ldots, a_{m-1}, a_{m}+\frac{x+z}{2}-1+c_{1}, c_{2}\right. \\
& \left.\quad \ldots, c_{k}, \frac{x+z}{2}+1, b_{n}, \ldots, b_{1}, y+a-\min (a, b)\right) \\
& \quad \times \frac{\mathrm{H}\left(c+\frac{x+z}{2}-1\right)}{\mathrm{H}(c) \mathrm{H}\left(\frac{x+z}{2}-1\right)} \frac{\mathrm{H}\left(\max (a, b)+y+\frac{x+z}{2}\right)}{\mathrm{H}\left(\max (a, b)+c+y+\frac{x+z}{2}\right)} \\
& \quad \times \frac{\mathrm{Hax}(a, b)+y+z) \mathrm{H}(\max (a, b)+c+y+z+1)}{\mathrm{H}\left(\max (a, b)-o_{a}+o_{b}+o_{c}+y+z+1\right) \mathrm{H}\left(\max (a, b)+o_{a}-o_{b}+e_{c}+y+z\right)} \\
& \quad \times \frac{\mathrm{H}\left(\max (a, b)-o_{a}+o_{b}+o_{c}+y+1\right) \mathrm{H}\left(\max (a, b)+o_{a}-o_{b}+e_{c}+y\right)}{\mathrm{H}(\max (a, b)+y+1) \mathrm{H}(\max (a, b)+y)},
\end{aligned}
$$

where $\Theta_{x, y, z}^{\prime}(m)$ is defined as in $\left(T^{\prime}-3\right)$.

Theorem 18. Assume that $\boldsymbol{a}=\left(a_{1}, a_{2}, \ldots, a_{m}\right), \boldsymbol{b}=\left(b_{1}, b_{2}, \ldots, b_{n}\right), \boldsymbol{c}=\left(c_{1}, c_{2}, \ldots, c_{k}\right)$ are three sequences of nonnegative integers ( $m, n, k$ are all even) and that $x, y, z$ are three integers, such that $x \geqslant 0, y \geqslant \max (a-b,-1), z \geqslant 0$, and $x$ and $z$ have the same parity. We have

$$
\begin{aligned}
& \left.\mathrm{M}\left(G_{x, y, z}^{(2)}(\boldsymbol{a} ; \boldsymbol{c} ; \boldsymbol{b})\right)=\Lambda_{2 y+z+2 \max (a, b)+1, z, x}^{\prime}(c)\right) \\
& \quad \times s\left(y+b-\min (a, b), a_{1}, \ldots, a_{m}, \frac{x+z}{2}-1, c_{1},\right. \\
& \left.\ldots, c_{k}+\frac{x+z}{2}+1+b_{n}, b_{n-1}, \ldots, b_{1}\right) \\
& \quad \times s\left(a_{1}, \ldots, a_{m-1}, a_{m}+\frac{x+z}{2}-1+c_{1}, c_{2},\right. \\
& \left.\quad \ldots, c_{k}, \frac{x+z}{2}+1, b_{n}, \ldots, b_{1}, y+a-\min (a, b)+1\right) \\
& \quad \times \frac{\mathrm{H}\left(c+\frac{x+z}{2}-1\right)}{\mathrm{H}(c) \mathrm{H}\left(\frac{x+z}{2}-1\right)} \frac{\mathrm{H}\left(\max (a, b)+y+\frac{x+z}{2}-1\right)}{\mathrm{H}\left(\max (a, b)+c+y+\frac{x+z}{2}-1\right)} \\
& \quad \times \frac{\mathrm{H}(\max (a, b)+y+z+1) \mathrm{H}(\max (a, b)+c+y+z)}{\mathrm{H}\left(\max (a, b)-o_{a}+o_{b}+o_{c}+y+z\right) \mathrm{H}\left(\max (a, b)+o_{a}-o_{b}+e_{c}+y+z+1\right)}
\end{aligned}
$$




$$
\times \frac{\mathrm{H}\left(\max (a, b)-o_{a}+o_{b}+o_{c}+y\right) \mathrm{H}\left(\max (a, b)+o_{a}-o_{b}+e_{c}+y+1\right)}{\mathrm{H}(\max (a, b)+y+1) \mathrm{H}(\max (a, b)+y)},
$$

where $\Lambda_{x, y, z}^{\prime}(m)$ is defined as in $\left(T^{\prime}-4\right)$.

Theorem 19. Assume that $\boldsymbol{a}=\left(a_{1}, a_{2}, \ldots, a_{m}\right), \boldsymbol{b}=\left(b_{1}, b_{2}, \ldots, b_{n}\right), \boldsymbol{c}=\left(c_{1}, c_{2}, \ldots, c_{k}\right)$ are three sequences of nonnegative integers ( $m, n, k$ are all even) and that $x, y, z$ are three integers, such that $x \geqslant 0, y \geqslant \max (a-b,-3), z \geqslant 0$, and $x$ and $z$ have the same parity. Then

$$
\begin{aligned}
& \mathrm{M}\left(G_{x, y, z}^{(3)}(\boldsymbol{a} ; \boldsymbol{c} ; \boldsymbol{b})\right)=\Psi_{2 y+z+2 \max (a, b)+3, z, x}^{\prime}(c) \\
& \quad \times s\left(y+b-\min (a, b), a_{1}, \ldots, a_{m}, \frac{x+z}{2}, c_{1}, \ldots, c_{k}+\frac{x+z}{2}+b_{n}, b_{n-1}, \ldots, b_{1}\right) \\
& \quad \times s\left(a_{1}, \ldots, a_{m-1}, a_{m}+\frac{x+z}{2}+c_{1}, \ldots, c_{k}, \frac{x+z}{2}, b_{n}, \ldots, b_{1}, y+a-\min (a, b)+3\right) \\
& \quad \times \frac{\mathrm{H}\left(c+\frac{x+z}{2}\right)}{\mathrm{H}(c) \mathrm{H}\left(\frac{x+z}{2}\right)} \frac{\mathrm{H}\left(\max (a, b)+y+\frac{x+z}{2}\right)}{\mathrm{H}\left(\max (a, b)+c+y+\frac{x+z}{2}\right)} \\
& \quad \times \frac{\mathrm{H}(\max (a, b)+y+z+3) \mathrm{H}(\max (a, b)+c+y+z)}{\mathrm{H}\left(\max (a, b)-o_{a}+o_{b}+o_{c}+y+z\right) \mathrm{H}\left(\max (a, b)+o_{a}-o_{b}+e_{c}+y+z+3\right)} \\
& \quad \times \frac{\mathrm{H}\left(\max (a, b)-o_{a}+o_{b}+o_{c}+y\right) \mathrm{H}\left(\max (a, b)+o_{a}-o_{b}+e_{c}+y+3\right)}{\mathrm{H}(\max (a, b)+y+3) \mathrm{H}(\max (a, b)+y)},
\end{aligned}
$$

where $\Psi_{x, y, z}^{\prime}(m)$ is defined as in (T'-2).

Theorem 20. Assume that $\boldsymbol{a}=\left(a_{1}, a_{2}, \ldots, a_{m}\right), \boldsymbol{b}=\left(b_{1}, b_{2}, \ldots, b_{n}\right), \boldsymbol{c}=\left(c_{1}, c_{2}, \ldots, c_{k}\right)$ are three sequences of nonnegative integers ( $m, n, k$ are all even) and that $x, y, z$ are three integers, such that $x \geqslant 0, y \geqslant \max (a-b,-3), z \geqslant 0$, and $x$ and $z$ have the same parity. We have

$$
\begin{aligned}
& \mathrm{M}\left(G_{x, y, z}^{(4)}(\boldsymbol{a} ; \boldsymbol{c} ; \boldsymbol{b})\right)=\Lambda_{z, 2 y+z+2 \max (a, b)+3, x}(c) \\
& \quad \times s\left(y+b-\min (a, b), a_{1}, \ldots, a_{m}, \frac{x+z}{2}+1, c_{1},\right. \\
& \left.\ldots, c_{k}+\frac{x+z}{2}+b_{n}-1, b_{n-1}, \ldots, b_{1}\right) \\
& \quad \times s\left(a_{1}, \ldots, a_{m-1}, a_{m}+\frac{x+z}{2}+c_{1}+1, c_{2},\right. \\
& \left.\ldots, c_{k}, \frac{x+z}{2}-1, b_{n}, \ldots, b_{1}, y+a-\min (a, b)+3\right) \\
& \quad \times \frac{\mathrm{H}\left(c+\frac{x+z}{2}+1\right)}{\mathrm{H}(c) \mathrm{H}\left(\frac{x+z}{2}+1\right)} \frac{\mathrm{H}\left(\max (a, b)+y+\frac{x+z}{2}+1\right)}{\mathrm{H}\left(\max (a, b)+c+y+\frac{x+z}{2}+1\right)}
\end{aligned}
$$




$$
\begin{aligned}
& \times \frac{\mathrm{H}(\max (a, b)+y+z+3) \mathrm{H}(\max (a, b)+c+y+z)}{\mathrm{H}\left(\max (a, b)-o_{a}+o_{b}+o_{c}+y+z\right) \mathrm{H}\left(\max (a, b)+o_{a}-o_{b}+e_{c}+y+z+3\right)} \\
& \times \frac{\mathrm{H}\left(\max (a, b)-o_{a}+o_{b}+o_{c}+y\right) \mathrm{H}\left(\max (a, b)+o_{a}-o_{b}+e_{c}+y+3\right)}{\mathrm{H}(\max (a, b)+y+3) \mathrm{H}(\max (a, b)+y)},
\end{aligned}
$$

where $\Lambda_{x, y, z}(m)$ is defined as in $(T-4)$.

\subsection{Off-central counterparts of the $R^{\swarrow}$-type regions}
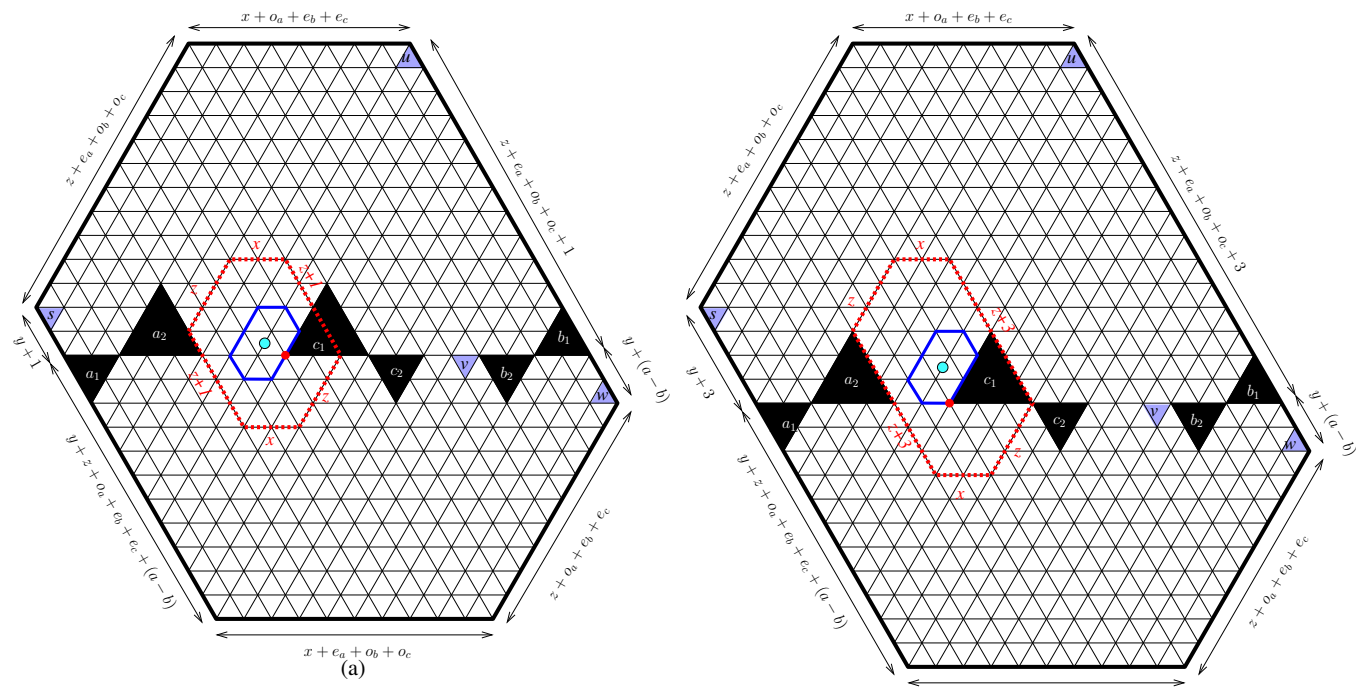

(b)

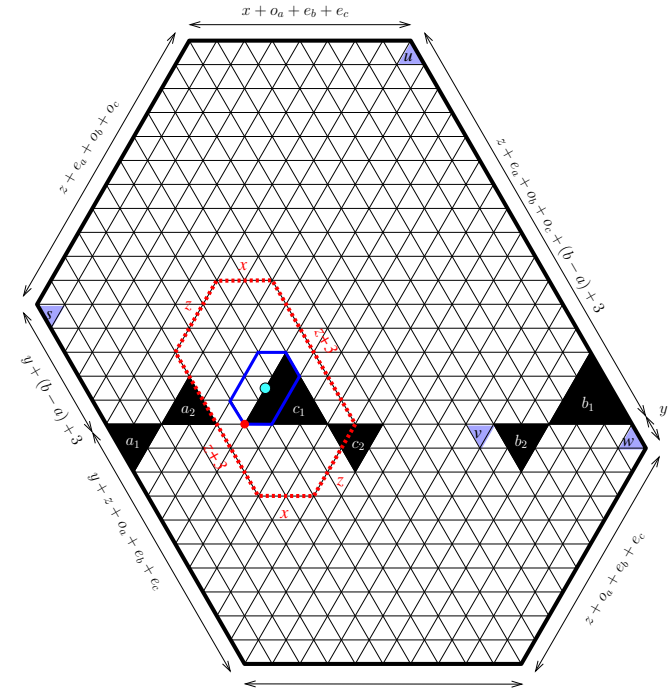

(c)

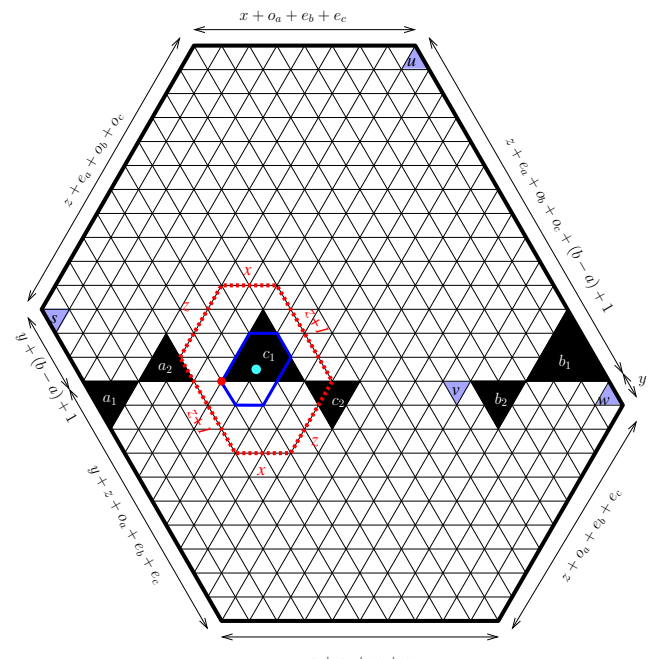

(d)

Figure 9: Four $K^{(i)}$-type regions: (a) $K_{2,1,3}^{(1)}(2,3 ; 3,2 ; 2,2)$, (b) $K_{2,1,3}^{(2)}(2,3 ; 3,2 ; 2,2)$, (c) $K_{2,1,3}^{(3)}(2,2 ; 3,2 ; 3,2)$, and (d) $K_{2,1,3}^{(4)}(2,2 ; 3,2 ; 3,2)$.

We consider the situation when the center of the auxiliary hexagon $H_{0}$ is the middle 
point of a southwest-to-northeast unit lattice interval. There are also eight off-central positions labeled by $1,2, \ldots, 8$ around the center as illustrated in Figure 4(d). Let $x$ and $z$ be two nonnegative integers with opposite parities. For $i=1,4,5,8$, we consider the auxiliary hexagon $H_{0}$ of side-lengths $x, z+1, z, x, z+1, z$, while for $i=2,3,6,7$, the auxiliary hexagon has side-lengths $x, z+3, z, x, z+3, z$. The domain of the $y$-parameter is determined by the level $d$ of the off-central position $i$ above or below the center of $H_{0}$ as in the definition of the $G^{(i)}$-type regions $(d=1 / 2$ or $3 / 2)$. Next, we apply the same side-pushing procedure as in the definition of the $E^{(i)}$-type regions and remove the three ferns at the same level, such that the middle fern has its root at the off-central position $i(i=1,2, \ldots, 8)$. Let us denote by $K_{x, y, z}^{(i)}(\mathbf{a} ; \mathbf{c} ; \mathbf{b})$ the newly defined region (see Figure 9 for examples). Again, by the symmetry, we only need to enumerate four of the eight regions, namely $K^{(1)}-, K^{(2)}-, K^{(3)}-$, and $K^{(4)}$-type regions.

Remark 21. In the definition of the $K^{(1)}$-type regions, if we remove the three ferns such that the root of the middle one is $1 / 2$-unit to the southwest of the center of the auxiliary hexagon $H_{0}$, then we obtain the region $R_{x, y, z}^{\swarrow}(\mathbf{a} ; \mathbf{c} ; \mathbf{b})$ in Theorem 2.5 of [31]. Therefore, we can view the $K^{(i)}$-type regions as off-central counterparts of the $R^{\swarrow}$-type regions.

Theorem 22. Assume that $\boldsymbol{a}=\left(a_{1}, a_{2}, \ldots, a_{m}\right), \boldsymbol{b}=\left(b_{1}, b_{2}, \ldots, b_{n}\right), \boldsymbol{c}=\left(c_{1}, c_{2}, \ldots, c_{k}\right)$ are three sequences of nonnegative integers ( $m, n, k$ are all even) and that $x, y, z$ are three nonnegative integers, such that $x \geqslant 0, y \geqslant \max (b-a,-1), z \geqslant 0$, and $x$ has parity opposite to z. Then

$$
\begin{aligned}
& \mathrm{M}\left(K_{x, y, z}^{(1)}(\boldsymbol{a} ; \boldsymbol{c} ; \boldsymbol{b})\right)=\Theta_{z, x, 2 y+z+2 \max (a, b)+1}^{\prime}(c) \\
& \quad \times s\left(y+b-\min (a, b)+1, a_{1}, \ldots, a_{m},\left\lceil\frac{x+z}{2}\right\rceil, c_{1},\right. \\
& \ldots, c_{k}+\left\lceil\frac{x+z}{2} \mid+b_{n}, b_{n-1}, \ldots, b_{1}\right) \\
& \times s\left(a_{1}, \ldots, a_{m-1}, a_{m}+\left\lceil\frac{x+z}{2}\right\rceil+c_{1}, c_{2}\right. \\
& \left.\left.\quad \ldots, c_{k}, \mid \frac{x+z}{2}\right\rfloor, b_{n}, \ldots, b_{1}, y+a-\min (a, b)\right) \\
& \quad \times \frac{\mathrm{H}\left(c+\left\lceil\frac{x+z}{2}\right\rceil\right)}{\mathrm{H}(c) \mathrm{H}\left(\left\lceil\frac{x+z}{2}\right\rceil\right)} \frac{\mathrm{H}\left(\max (a, b)+y+\left\lceil\frac{x+z}{2}\right\rceil+1\right)}{\mathrm{H}\left(\max (a, b)+c+y+\left\lceil\frac{x+z}{2}\right\rceil+1\right)} \\
& \quad \times \frac{\mathrm{Hax}(a, b)+y+z) \mathrm{H}(\max (a, b)+c+y+z+1)}{\mathrm{H}\left(\max (a, b)-o_{a}+o_{b}+o_{c}+y+z+1\right) \mathrm{H}\left(\max (a, b)+o_{a}-o_{b}+e_{c}+y+z\right)} \\
& \quad \times \frac{\mathrm{H}\left(\max (a, b)-o_{a}+o_{b}+o_{c}+y+1\right) \mathrm{H}\left(\max (a, b)+o_{a}-o_{b}+e_{c}+y\right)}{\mathrm{H}(\max (a, b)+y+1) \mathrm{H}(\max (a, b)+y)},
\end{aligned}
$$

where the region $\Theta_{x, y, z}^{\prime}(m)$ is defined as in $\left(T^{\prime}-3\right)$.

Theorem 23. Assume that $\boldsymbol{a}=\left(a_{1}, a_{2}, \ldots, a_{m}\right), \boldsymbol{b}=\left(b_{1}, b_{2}, \ldots, b_{n}\right), \boldsymbol{c}=\left(c_{1}, c_{2}, \ldots, c_{k}\right)$ are three sequences of nonnegative integers ( $m, n, k$ are all even) and that $x, y, z$ are three 
integers, such that $x \geqslant 0, y \geqslant \max (b-a,-3), z \geqslant 0$, and $x$ has parity opposite to $z$. Then

$$
\begin{aligned}
& \mathrm{M}\left(K_{x, y, z}^{(2)}(\boldsymbol{a} ; \boldsymbol{c} ; \boldsymbol{b})\right)=\Lambda_{z, x, 2 y+z+2 \max (a, b)+3}^{\prime}(c) \\
& \quad \times s\left(y+b-\min (a, b)+3, a_{1}, \ldots, a_{m},\left\lfloor\frac{x+z}{2}\right\rfloor, c_{1},\right. \\
& \left.\ldots, c_{k}+\left\lceil\frac{x+z}{2}\right\rceil+b_{n}, b_{n-1}, \ldots, b_{1}\right) \\
& \times s\left(a_{1}, \ldots, a_{m-1}, a_{m}+\left\lfloor\frac{x+z}{2}\right\rfloor+c_{1}, c_{2},\right. \\
& \left.\quad \ldots, c_{k},\left\lceil\frac{x+z}{2}\right\rceil, b_{n}, \ldots, b_{1}, y+a-\min (a, b)\right) \\
& \quad \times \frac{\mathrm{H}\left(c+\left\lfloor\frac{x+z}{2}\right\rfloor\right)}{\mathrm{H}(c) \mathrm{H}\left(\left\lfloor\frac{x+z}{2}\right\rfloor\right)} \frac{\mathrm{H}\left(\max (a, b)+y+\left\lceil\frac{x+z}{2}\right\rceil+2\right)}{\mathrm{H}\left(\max (a, b)+c+y+\left\lceil\frac{x+z}{2}\right\rceil+2\right)} \\
& \quad \times \frac{\mathrm{max}(a, b)+y+z) \mathrm{H}(\max (a, b)+c+y+z+3)}{\mathrm{H}\left(\max (a, b)-o_{a}+o_{b}+o_{c}+y+z+3\right) \mathrm{H}\left(\max (a, b)+o_{a}-o_{b}+e_{c}+y+z\right)} \\
& \quad \times \frac{\mathrm{H}\left(\max (a, b)-o_{a}+o_{b}+o_{c}+y+3\right) \mathrm{H}\left(\max (a, b)+o_{a}-o_{b}+e_{c}+y\right)}{\mathrm{H}(\max (a, b)+y+3) \mathrm{H}(\max (a, b)+y)},
\end{aligned}
$$

where the region $\Lambda_{x, y, z}^{\prime}(m)$ is defined as in $\left(T^{\prime}-4\right)$.

Theorem 24. Assume that $\boldsymbol{a}=\left(a_{1}, a_{2}, \ldots, a_{m}\right), \boldsymbol{b}=\left(b_{1}, b_{2}, \ldots, b_{n}\right), \boldsymbol{c}=\left(c_{1}, c_{2}, \ldots, c_{k}\right)$ are three sequences of nonnegative integers $(m, n, k$ are all even) and that $x, y, z$ are three integers, such that $x \geqslant 0, y \geqslant \max (b-a,-3), z \geqslant 0$, and $x$ has parity opposite to $x$. We get

$$
\begin{aligned}
& \mathrm{M}\left(K_{x, y, z}^{(3)}(\boldsymbol{a} ; \boldsymbol{c} ; \boldsymbol{b})\right)=\Psi_{z, x, 2 y+z+2 \max (a, b)+3}^{\prime}(c) \\
& \times s\left(y+b-\min (a, b)+3, a_{1}, \ldots, a_{m},\left\lfloor\frac{x+z}{2}\right\rfloor-1, c_{1},\right. \\
& \left.\ldots, c_{k}+\left\lceil\frac{x+z}{2}\right\rceil+1+b_{n}, b_{n-1}, \ldots, b_{1}\right) \\
& \times s\left(a_{1}, \ldots, a_{m-1}, a_{m}+\left\lfloor\frac{x+z}{2}\right\rfloor-1+c_{1}, c_{2}\right. \\
& \left.\ldots, c_{k},\left\lceil\frac{x+z}{2}\right\rceil+1, b_{n}, \ldots, b_{1}, y+a-\min (a, b)\right) \\
& \times \frac{\mathrm{H}\left(c+\left\lfloor\frac{x+z}{2}\right\rfloor-1\right)}{\mathrm{H}(c) \mathrm{H}\left(\left\lfloor\frac{x+z}{2}\right\rfloor-1\right)} \frac{\mathrm{H}\left(\max (a, b)+y+\left\lceil\frac{x+z}{2}\right\rceil+1\right)}{\mathrm{H}\left(\max (a, b)+c+y+\left\lceil\frac{x+z}{2}\right\rceil+1\right)} \\
& \times \frac{\mathrm{H}(\max (a, b)+y+z) \mathrm{H}(\max (a, b)+c+y+z+3)}{\mathrm{H}\left(\max (a, b)-o_{a}+o_{b}+o_{c}+y+z+3\right) \mathrm{H}\left(\max (a, b)+o_{a}-o_{b}+e_{c}+y+z\right)} \\
& \times \frac{\mathrm{H}\left(\max (a, b)-o_{a}+o_{b}+o_{c}+y+3\right) \mathrm{H}\left(\max (a, b)+o_{a}-o_{b}+e_{c}+y\right)}{\mathrm{H}(\max (a, b)+y+3) \mathrm{H}(\max (a, b)+y)},
\end{aligned}
$$


where the region $\Psi_{x, y, z}^{\prime}(m)$ is defined as in (T'-2).

Theorem 25. Assume that $\boldsymbol{a}=\left(a_{1}, a_{2}, \ldots, a_{m}\right), \boldsymbol{b}=\left(b_{1}, b_{2}, \ldots, b_{n}\right), \boldsymbol{c}=\left(c_{1}, c_{2}, \ldots, c_{k}\right)$ are three sequences of nonnegative integers ( $m, n, k$ are all even) and that $x, y, z$ are three integers, such that $x \geqslant 0, y \geqslant \max (b-a,-1), z \geqslant 0$, and $x$ has parity opposite to $z$. Then

$$
\begin{aligned}
& \mathrm{M}\left(K_{x, y, z}^{(4)}(\boldsymbol{a} ; \boldsymbol{c} ; \boldsymbol{b})\right)=\Lambda_{2 y+z+2 \max (a, b)+1, x, z}(c) \\
& \times s\left(y+b-\min (a, b)+1, a_{1}, \ldots, a_{m},\left\lfloor\frac{x+z}{2}\right\rfloor-1, c_{1},\right. \\
& \left.\ldots, c_{k}+\left\lceil\frac{x+z}{2}\right\rceil+b_{n}+1, b_{n-1}, \ldots, b_{1}\right) \\
& \times s\left(a_{1}, \ldots, a_{m-1}, a_{m}+\left\lfloor\frac{x+z}{2}\right\rfloor+c_{1}-1, c_{2},\right. \\
& \left.\ldots, c_{k},\left\lceil\frac{x+z}{2}\right\rceil+1, b_{n}, \ldots, b_{1}, y+a-\min (a, b)\right) \\
& \times \frac{\mathrm{H}\left(c+\left\lfloor\frac{x+z}{2}\right\rfloor-1\right)}{\mathrm{H}(c) \mathrm{H}\left(\left\lfloor\frac{x+z}{2}\right\rfloor-1\right)} \frac{\mathrm{H}\left(\max (a, b)+y+\left\lfloor\frac{x+z}{2}\right\rfloor\right)}{\mathrm{H}\left(\max (a, b)+c+y+\left\lfloor\frac{x+z}{2}\right\rfloor\right)} \\
& \times \frac{\mathrm{H}(\max (a, b)+y+z) \mathrm{H}(\max (a, b)+c+y+z+1)}{\mathrm{H}\left(\max (a, b)-o_{a}+o_{b}+o_{c}+y+z+1\right) \mathrm{H}\left(\max (a, b)+o_{a}-o_{b}+e_{c}+y+z\right)} \\
& \times \frac{\mathrm{H}\left(\max (a, b)-o_{a}+o_{b}+o_{c}+y+1\right) \mathrm{H}\left(\max (a, b)+o_{a}-o_{b}+e_{c}+y\right)}{\mathrm{H}(\max (a, b)+y+1) \mathrm{H}(\max (a, b)+y)},
\end{aligned}
$$

where the region $\Lambda_{x, y, z}(m)$ is defined as in $(T-4)$.

\subsection{The $\bar{E}^{(i)}$-type regions}

In the previous four families of off-central regions, the horizontal line $l$, which contains the three removed ferns, separates the east and the west vertices of base hexagon $H$. We next consider the other situation when the line $l$ leaves the west and the east vertices of the base hexagon on the same side. Without loss of generality, we assume that these vertices are both below the line $l$.

Similar to the cases of the previous four off-central families, we define our next region as follows. We (1) start with a certain auxiliary hexagon $H_{0}$ of certain side-lengths, $(2)$ perform an edge-pushing procedure on the sides of $H_{0}$ to get the base hexagon $H$, and (3) remove three ferns from the resulting base hexagon at the same level, such that the leftmost point of the middle fern is at one of the off-central positions as shown in Figure 4 and that the left and the right ferns touch the northwest and the northeast sides of the base hexagon. The only major difference is that, in step (2), we will perform a different pushing procedure. In particular, we now push respectively the north, northeast, southeast, south, southwest, northwest sides of $H_{0}$ a distance of $o_{a}+o_{b}+o_{c}, b+c, b+c+y+\max (0, a-b)$, $e_{a}+e_{b}+e_{c}+2 y+|a-b|, a+y+\max (0, b-a), a$ units. This procedure is illustrated in Figure 10 . 


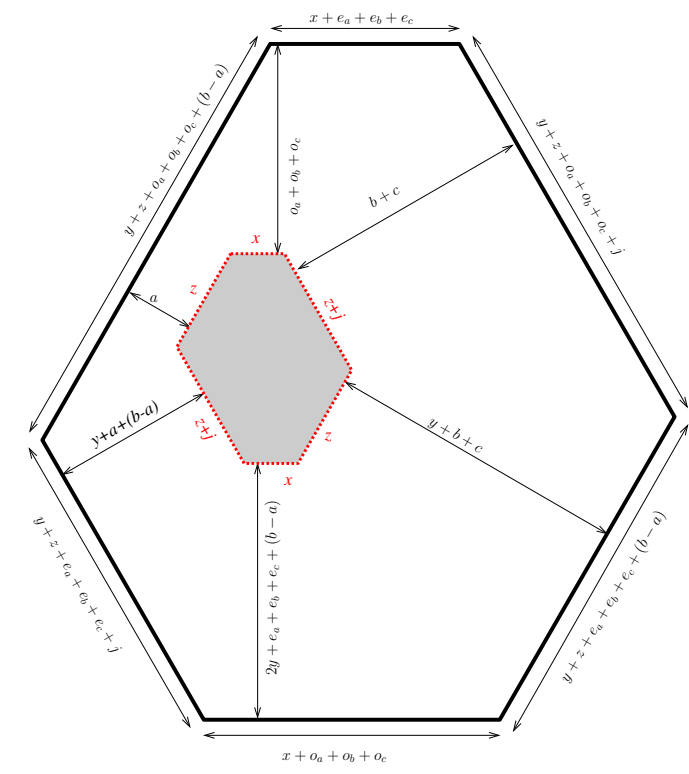

(a)

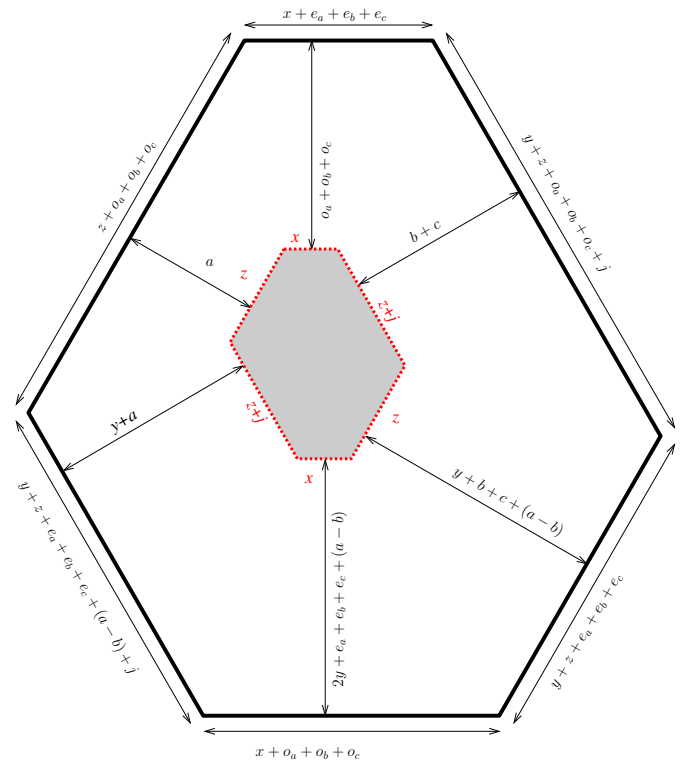

(b)

Figure 10: The edge-pushing procedure used in the definitions of the $\bar{E}^{(i)}-, \bar{F}^{(i)}{ }_{-}, \bar{G}^{(i)}{ }_{-}$, $\bar{K}^{(i)}$-type regions: (a) the case $a \leqslant b$, (b) the case $a \geqslant b$.

Let $x, z$ be two nonnegative integers of the same parity, and let $y$ be an integer that may be negative as indicated particularly in the statements of the theorems below. For $i=1$ or 4 , we perform the above pushing procedure on the auxiliary hexagon $H_{0}$ of sidelengths $x, z, z, x, z, z$. In this way, we get the base hexagon $H$ of side-lengths $x+e_{a}+e_{b}+$ $e_{c}, y+z+o_{a}+o_{b}+o_{c}+\max (a-b, 0), y+z+e_{a}+e_{b}+e_{c}+\max (b-a, 0), x+o_{a}+o_{b}+o_{c}, y+$ $z+e_{a}+e_{b}+e_{c}+\max (a-b, 0), y+z+o_{a}+o_{b}+o_{c}+\max (b-a, 0)$. Denote by $\bar{E}^{(i)}$ the region corresponding to the choice of the middle fern whose leftmost is at the off-central position $i$ in Figure 4(a), for $i=1,4$. We do the same in the case $i=2,3$; the only difference is that we start with an auxiliary hexagon of side-lengths $x, z+2, z, x, z+2, z$ (as opposed to the one of side-lengths $x, z, z, x, z, z)$. See Figure 11 for examples.

We note that in this family (and in the following three families), we will only allow the root of the middle fern to be above or on the same level as the center of the auxiliary hexagon $H_{0}$. In particular, our region here is not defined when $i=5,6$.

Again, by symmetries, it is enough for us to enumerate only three of the four new regions, namely $\bar{E}^{(1)}-, \bar{E}^{(2)}-, \bar{E}^{(3)}$-type regions, as any $\bar{E}^{(4)}$-type region is simply a reflection of some $\bar{E}^{(1)}$-type one over a vertical line.

Remark 26. In the definition of the $\bar{E}^{(1)}$-type regions, if we remove the three ferns such that the root of the middle one is at the center of the auxiliary hexagon $H_{0}$, we obtain the region $Q_{x, y, z}^{\odot}(\mathbf{a} ; \mathbf{c} ; \mathbf{b})$ in Theorem 2.5 of [31]. In some sense, the $\bar{E}^{(i)}$-type regions can be viewed as off-central counterparts of the $Q^{\odot}$-type regions. 


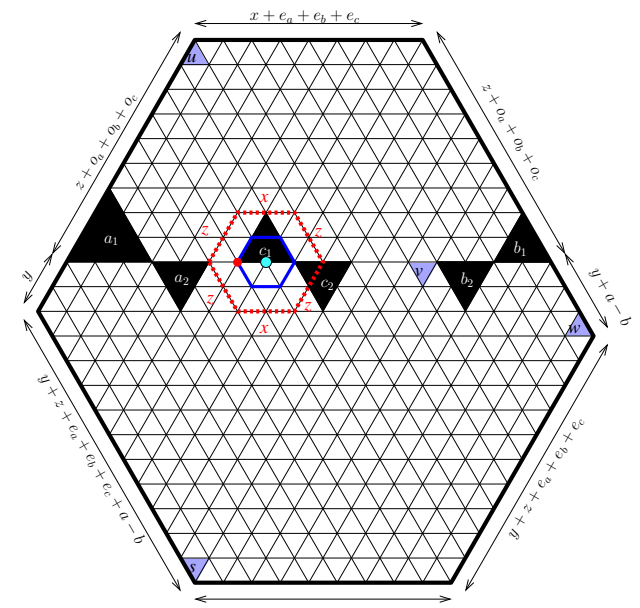

(a)

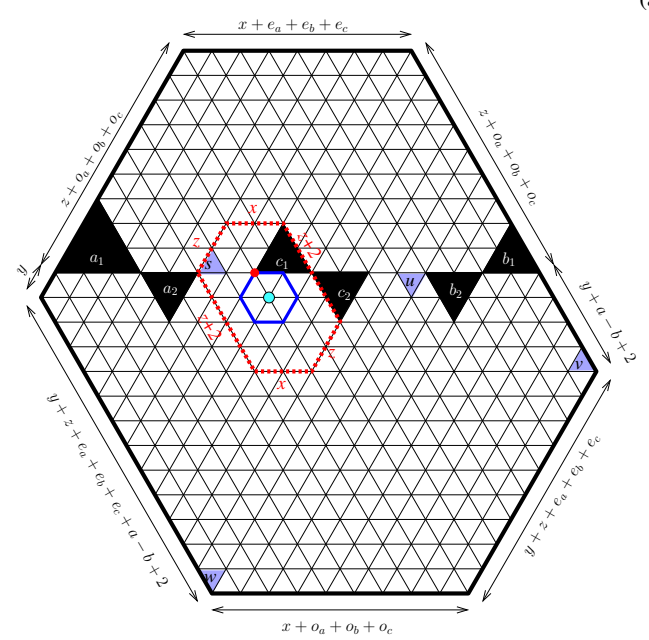

(b)

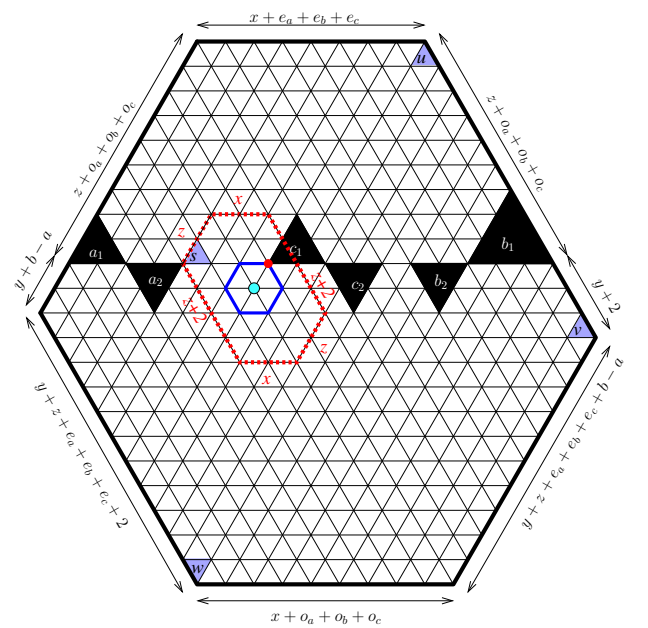

(c)

Figure 11: Three $\bar{E}^{(i)}$-type regions: (a) $\bar{E}_{2,2,2}^{(1)}(3,2 ; 2,2 ; 2,2)$, (b) $\bar{E}_{2,1,2}^{(2)}(3,2 ; 2,2 ; 2,2)$, and (c) $\bar{E}_{2,1,2}^{(3)}(2,2 ; 2,2 ; 3,2)$.

Theorem 27. Assume that $\boldsymbol{a}=\left(a_{1}, a_{2}, \ldots, a_{m}\right), \boldsymbol{b}=\left(b_{1}, b_{2}, \ldots, b_{n}\right), \boldsymbol{c}=\left(c_{1}, c_{2}, \ldots, c_{k}\right)$ are three sequences of nonnegative integers ( $m, n, k$ are all even) and that $x, y, z$ are three nonnegative integers, such that $x$ and $z$ have the same parity. Then

$$
\begin{aligned}
& \mathrm{M}\left(\bar{E}_{x, y, z}^{(1)}(\boldsymbol{a} ; \boldsymbol{c} ; \boldsymbol{b})\right)=\Phi_{x, 2 y+z+2 \max (a, b), z}(c) \\
& \times s\left(a_{1}, \ldots, a_{m}+\frac{x+z}{2}-1, c_{1}, \ldots, c_{k}+\frac{x+z}{2}+b_{n}+1, b_{n-1}, \ldots, b_{1}\right) \\
& \times s\left(y+b-\min (a, b), a_{1}, \ldots, a_{m}, \frac{x+z}{2}+c_{1}-1, c_{2},\right. \\
& \left.\ldots, c_{k}, \frac{x+z}{2}+1, b_{n}, \ldots, b_{1}, y+a-\min (a, b)\right)
\end{aligned}
$$




$$
\begin{aligned}
& \times \frac{\mathrm{H}\left(c+\frac{x+z}{2}-1\right)}{\mathrm{H}(c) \mathrm{H}\left(\frac{x+z}{2}-1\right)} \frac{\mathrm{H}\left(\max (a, b)+y+\frac{x+z}{2}-1\right)}{\mathrm{H}\left(\max (a, b)+c+y+\frac{x+z}{2}-1\right)} \\
& \times \frac{\mathrm{H}(\max (a, b)+y+z) \mathrm{H}(\max (a, b)+c+y+z)}{\mathrm{H}\left(o_{a}+o_{b}+o_{c}+z\right) \mathrm{H}\left(|a-b|+e_{a}+e_{b}+e_{c}+2 y+z\right)} \\
& \times \frac{\mathrm{H}\left(o_{a}+o_{b}+o_{c}\right) \mathrm{H}\left(|a-b|+e_{a}+e_{b}+e_{c}+2 y\right)}{\mathrm{H}(\max (a, b)+y)^{2}},
\end{aligned}
$$

where $\Phi_{x, y, z}(m)$ denotes the formula in (T-1) in Conjecture 1.

Theorem 28. Assume that $\boldsymbol{a}=\left(a_{1}, a_{2}, \ldots, a_{m}\right), \boldsymbol{b}=\left(b_{1}, b_{2}, \ldots, b_{n}\right), \boldsymbol{c}=\left(c_{1}, c_{2}, \ldots, c_{k}\right)$ are three sequences of nonnegative integers ( $m, n, k$ are all even) and that $x, y, z$ are three integers, such that $x \geqslant 0, y \geqslant \max (a-b,-2), z \geqslant 0$, and $x$ and $z$ have the same parity. Then

$$
\begin{aligned}
\mathrm{M} & \left(\bar{E}_{x, y, z}^{(2)}(\boldsymbol{a} ; \boldsymbol{c} ; \boldsymbol{b})\right)=\Phi_{2 y+z+2 \max (a, b)+2, x, z}(c) \\
& \times s\left(a_{1}, \ldots, a_{m}+\frac{x+z}{2}, c_{1}, \ldots, c_{k}+\frac{x+z}{2}+b_{n}, b_{n-1}, \ldots, b_{1}\right) \\
& \times s\left(y+b-\min (a, b), a_{1}, \ldots, a_{m}, \frac{x+z}{2}+c_{1}, c_{2},\right. \\
& \left.\ldots, c_{k}, \frac{x+z}{2}, b_{n}, \ldots, b_{1}, y+a-\min (a, b)+2\right) \\
& \times \frac{\mathrm{H}\left(c+\frac{x+z}{2}\right)}{\mathrm{H}(c) \mathrm{H}\left(\frac{x+z}{2}\right)} \frac{\mathrm{H}\left(\max (a, b)+y+\frac{x+z}{2}\right)}{\mathrm{H}\left(\max (a, b)+c+y+\frac{x+z}{2}\right)} \\
& \times \frac{\mathrm{H}(\max (a, b)+y+z+2) \mathrm{H}(\max (a, b)+c+y+z)}{\mathrm{H}\left(o_{a}+o_{b}+o_{c}+z\right) \mathrm{H}\left(|a-b|+e_{a}+e_{b}+e_{c}+2 y+z+2\right)} \\
& \times \frac{\mathrm{H}\left(o_{a}+o_{b}+o_{c}\right) \mathrm{H}\left(|a-b|+e_{a}+e_{b}+e_{c}+2 y+2\right)}{\mathrm{H}(\max (a, b)+y) \mathrm{H}(\max (a, b)+y+2)} .
\end{aligned}
$$

Theorem 29. Assume that $\boldsymbol{a}=\left(a_{1}, a_{2}, \ldots, a_{m}\right), \boldsymbol{b}=\left(b_{1}, b_{2}, \ldots, b_{n}\right), \boldsymbol{c}=\left(c_{1}, c_{2}, \ldots, c_{k}\right)$ are three sequences of nonnegative integers ( $m, n, k$ are all even) and that $x, y, z$ are three integers, such that $x \geqslant 0, y \geqslant \max (a-b,-2), z \geqslant 0$, and $x$ and $z$ have the same parity. Then

$$
\begin{aligned}
& \mathrm{M}\left(\bar{E}_{x, y, z}^{(3)}(\boldsymbol{a} ; \boldsymbol{c} ; \boldsymbol{b})\right)=\Phi_{z, x, 2 y+z+2 \max (a, b)+2}(c) \\
& \quad \times s\left(a_{1}, \ldots, a_{m}+\frac{x+z}{2}+1, c_{1}, \ldots, c_{k}+\frac{x+z}{2}+b_{n}-1, b_{n-1}, \ldots, b_{1}\right) \\
& \quad \times s\left(y+b-\min (a, b), a_{1}, \ldots, a_{m}, \frac{x+z}{2}+c_{1}+1, c_{2},\right. \\
& \left.\quad \ldots, c_{k}, \frac{x+z}{2}-1, b_{n}, \ldots, b_{1}, y+a-\min (a, b)+2\right) \\
& \quad \times \frac{\mathrm{H}\left(c+\frac{x+z}{2}-1\right)}{\mathrm{H}(c) \mathrm{H}\left(\frac{x+z}{2}-1\right)} \frac{\mathrm{H}\left(\max (a, b)+y+\frac{x+z}{2}+1\right)}{\mathrm{H}\left(\max (a, b)+c+y+\frac{x+z}{2}+1\right)}
\end{aligned}
$$




$$
\begin{aligned}
& \times \frac{\mathrm{H}(\max (a, b)+y+z) \mathrm{H}(\max (a, b)+c+y+z+2)}{\mathrm{H}\left(o_{a}+o_{b}+o_{c}+z\right) \mathrm{H}\left(|a-b|+e_{a}+e_{b}+e_{c}+2 y+z+2\right)} \\
& \times \frac{\mathrm{H}\left(o_{a}+o_{b}+o_{c}\right) \mathrm{H}\left(|a-b|+e_{a}+e_{b}+e_{c}+2 y+2\right)}{\mathrm{H}(\max (a, b)+y) \mathrm{H}(\max (a, b)+y+2)} .
\end{aligned}
$$

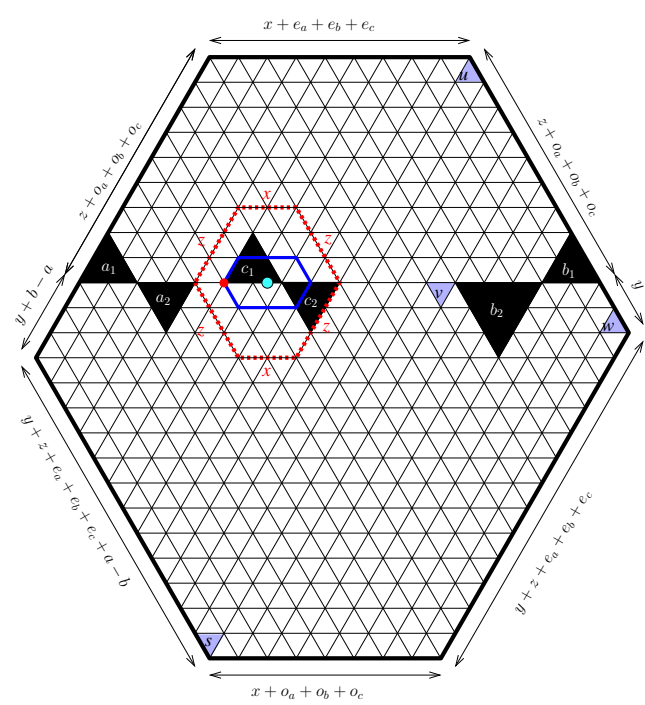

(a)

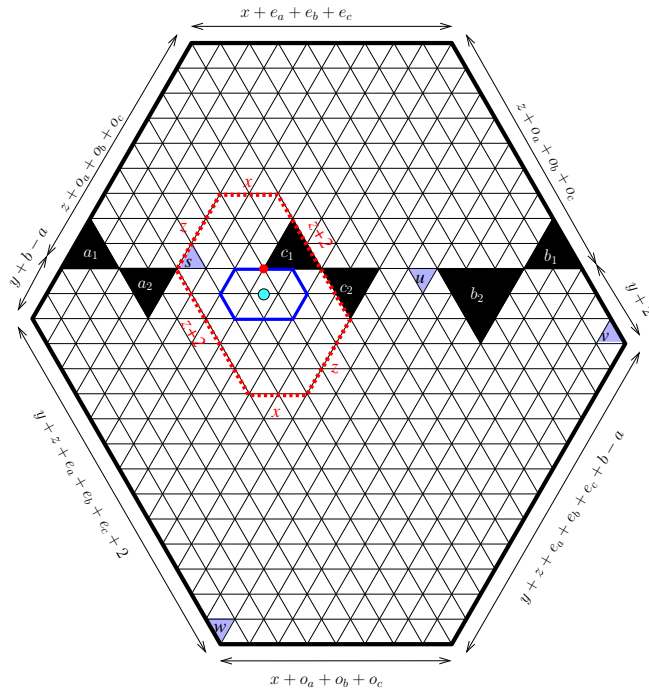

(c)

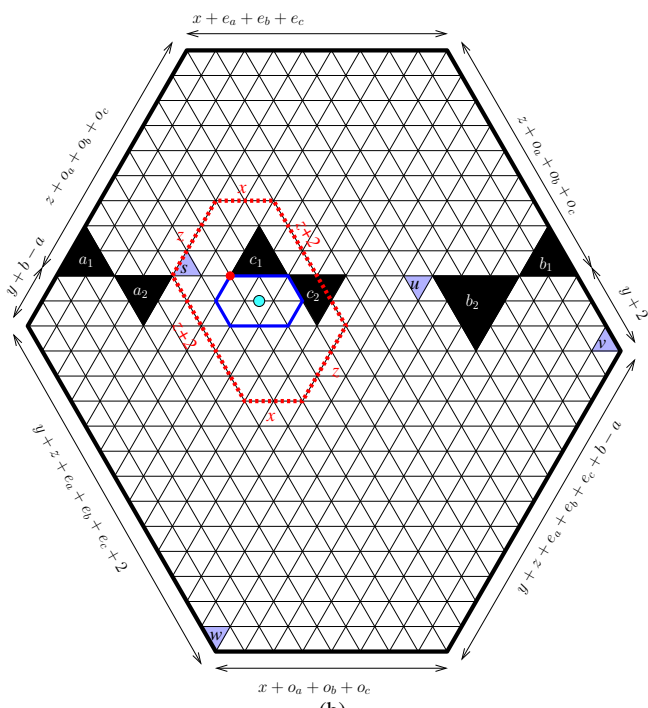

(b)

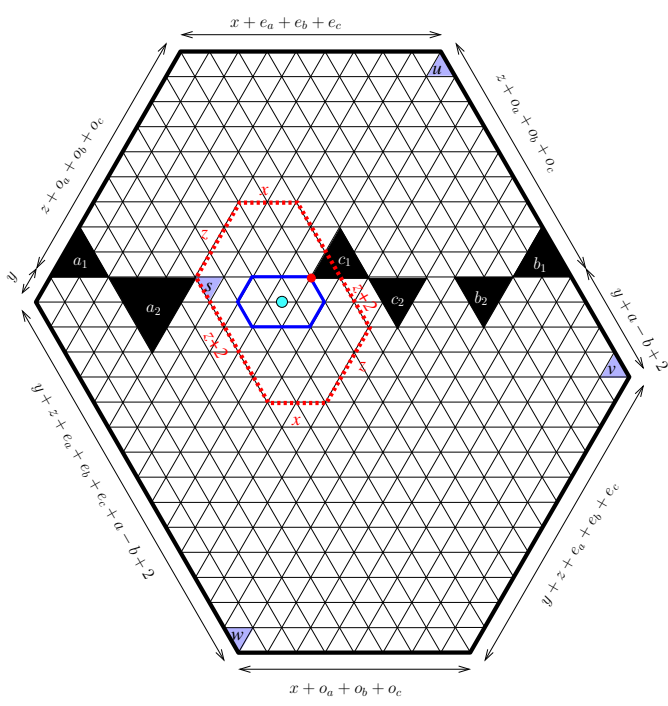

(d)

Figure 12: Four $\bar{F}^{(i)}$-type regions: (a) $\bar{F}_{2,2,3}^{(3)}(2,2 ; 2,2 ; 2,3)$, (b) $\bar{F}_{2,1,3}^{(4)}(2,2 ; 2,2 ; 2,3)$, (c) $\bar{F}_{2,1,3}^{(5)}(2,2 ; 2,2 ; 2,3)$, and $(\mathrm{d}) \bar{F}_{2,1,3}^{(6)}(2,3 ; 2,2 ; 2,2)$. 


\subsection{The $\bar{F}^{(i)}$-type regions}

We now consider the case when $x$ and $z$ have opposite parities. For $i=4,5,6$, we start with an auxiliary hexagon $H_{0}$ of side-lengths $x, z+2, z, x, z+2, z$, and for $i=3$, 7 , we start with the auxiliary hexagon of side-lengths $x, z, z, x, z, z$. We still push all six sides of the auxiliary hexagon as in the definition of the $\bar{E}^{(i)}$-type regions to get the base hexagon $H$, and remove the three ferns from the resulting hexagon $H$, such that the root of the middle fern is now at the off-central position $i$ as shown in Figure 4(b) (the left and right ferns are also determined by the position of the middle one). Similar to the case of the $\bar{E}^{(i)}$-type regions, there are no counterparts for the case $i=1,2,8$. Denote by $\bar{F}_{x, y, z}^{(i)}(\mathbf{a} ; \mathbf{c} ; \mathbf{b})$ the resulting regions (illustrated in Figure 12). By the symmetry, we only need to enumerate four of the five regions (as any $\bar{F}^{(7)}$-type region is a vertical reflection of an $\bar{F}^{(3)}$-type one). Remark 30. In the definition of the $\bar{F}^{(3)}$-type regions, if we remove the three ferns such that the root of the middle one is $1 / 2$-unit to the left of the center of the auxiliary hexagon $H_{0}$, then we obtain the region $Q_{x, y, z}^{\leftarrow}(\mathbf{a} ; \mathbf{c} ; \mathbf{b})$ in Theorem 2.6 of [31].

Theorem 31. Assume that $\boldsymbol{a}=\left(a_{1}, a_{2}, \ldots, a_{m}\right), \boldsymbol{b}=\left(b_{1}, b_{2}, \ldots, b_{n}\right), \boldsymbol{c}=\left(c_{1}, c_{2}, \ldots, c_{k}\right)$ are three sequences of nonnegative integers ( $m, n, k$ are all even) and that $x, y, z$ are three nonnegative integers, such that $x$ and $z$ have the different parities. Then

$$
\begin{aligned}
\mathrm{M} & \left(\bar{F}_{x, y, z}^{(3)}(\boldsymbol{a} ; \boldsymbol{c} ; \boldsymbol{b})\right)=\Psi_{z, 2 y+z+2 \max (a, b), x}(c) \\
& \times s\left(a_{1}, \ldots, a_{m}+\left\lfloor\frac{x+z}{2}\right\rfloor-1, c_{1}, \ldots, c_{k}+\left\lceil\frac{x+z}{2}\right\rceil+b_{n}+1, b_{n-1}, \ldots, b_{1}\right) \\
& \times s\left(y+b-\min (a, b), a_{1}, \ldots, a_{m},\left\lfloor\frac{x+z}{2}\right\rfloor+c_{1}-1, c_{2},\right. \\
& \left.\ldots, c_{k},\left\lceil\frac{x+z}{2}\right\rceil+1, b_{n}, \ldots, b_{1}, y+a-\min (a, b)\right) \\
& \times \frac{\mathrm{H}\left(c+\left\lfloor\frac{x+z}{2}\right\rfloor-1\right)}{\mathrm{H}(c) \mathrm{H}\left(\left\lfloor\frac{x+z}{2}\right\rfloor-1\right)} \frac{\mathrm{H}\left(\max (a, b)+y+\left\lfloor\frac{x+z}{2}\right\rfloor-1\right)}{\mathrm{H}\left(\max (a, b)+c+y+\left\lfloor\frac{x+z}{2}\right\rfloor-1\right)} \\
& \times \frac{\mathrm{H}(\max (a, b)+y+z) \mathrm{H}(\max (a, b)+c+y+z)}{\mathrm{H}\left(o_{a}+o_{b}+o_{c}+z\right) \mathrm{H}\left(|a-b|+e_{a}+e_{b}+e_{c}+2 y+z\right)} \\
& \times \frac{\mathrm{H}\left(o_{a}+o_{b}+o_{c}\right) \mathrm{H}\left(|a-b|+e_{a}+e_{b}+e_{c}+2 y\right)}{\mathrm{H}(\max (a, b)+y)^{2}}
\end{aligned}
$$

where $\Psi_{x, y, z}(m)$ denotes the formula in (T-2).

Theorem 32. Assume that $\boldsymbol{a}=\left(a_{1}, a_{2}, \ldots, a_{m}\right), \boldsymbol{b}=\left(b_{1}, b_{2}, \ldots, b_{n}\right), \boldsymbol{c}=\left(c_{1}, c_{2}, \ldots, c_{k}\right)$ are three sequences of nonnegative integers ( $m, n, k$ are all even) and that $x, y, z$ are three integers, such that $x \geqslant 0, y \geqslant \max (a-b,-2), z \geqslant 0$, and $x$ and $z$ have different parities. Then 


$$
\begin{aligned}
& \mathrm{M}\left(\bar{F}_{x, y, z}^{(4)}(\boldsymbol{a} ; \boldsymbol{c} ; \boldsymbol{b})\right)=\Lambda_{x, z, 2 y+z+2 \max (a, b)+2}^{\prime}(c) \\
& \quad \times s\left(a_{1}, \ldots, a_{m}+\left\lfloor\frac{x+z}{2}\right\rfloor, c_{1}, \ldots, c_{k}+\left\lceil\frac{x+z}{2}\right\rceil+b_{n}, b_{n-1}, \ldots, b_{1}\right) \\
& \quad \times s\left(y+b-\min (a, b), a_{1}, \ldots, a_{m},\left\lfloor\frac{x+z}{2}\right\rfloor+c_{1}, c_{2},\right. \\
& \left.\quad \ldots, c_{k},\left\lceil\frac{x+z}{2}\right\rfloor, b_{n}, \ldots, b_{1}, y+a-\min (a, b)+2\right) \\
& \quad \times \frac{\mathrm{H}\left(c+\left\lfloor\frac{x+z}{2}\right\rfloor\right)}{\mathrm{H}(c) \mathrm{H}\left(\left\lfloor\frac{x+z}{2}\right\rfloor\right)} \frac{\mathrm{H}\left(\max (a, b)+y+\left\lfloor\frac{x+z}{2}\right\rfloor\right)}{\mathrm{H}\left(\max (a, b)+c+y+\left\lfloor\frac{x+z}{2}\right\rfloor\right)} \\
& \quad \times \frac{\mathrm{H}(\max (a, b)+y+z+2) \mathrm{H}(\max (a, b)+c+y+z)}{\mathrm{H}\left(o_{a}+o_{b}+o_{c}+z\right) \mathrm{H}\left(|a-b|+e_{a}+e_{b}+e_{c}+2 y+z+2\right)} \\
& \quad \times \frac{\mathrm{H}\left(o_{a}+o_{b}+o_{c}\right) \mathrm{H}\left(|a-b|+e_{a}+e_{b}+e_{c}+2 y+2\right)}{\mathrm{H}(\max (a, b)+y) \mathrm{H}(\max (a, b)+y+2)},
\end{aligned}
$$

where $\Lambda_{x, y, z}^{\prime}(m)$ is defined as in $\left(T^{\prime}-4\right)$.

Theorem 33. Assume that $\boldsymbol{a}=\left(a_{1}, a_{2}, \ldots, a_{m}\right), \boldsymbol{b}=\left(b_{1}, b_{2}, \ldots, b_{n}\right), \boldsymbol{c}=\left(c_{1}, c_{2}, \ldots, c_{k}\right)$ are three sequences of nonnegative integers ( $m, n, k$ are all even) and that $x, y, z$ are three integers, such that $x \geqslant 0, y \geqslant \max (a-b,-2), z \geqslant 0$, and $x$ and $z$ have different parities. Then

$$
\begin{aligned}
& \mathrm{M}\left(\bar{F}_{x, y, z}^{(5)}(\boldsymbol{a} ; \boldsymbol{c} ; \boldsymbol{b})\right)=\Theta_{x, 2 y+z+2 \max (a, b)+2, z}^{\prime}(c) \\
& \quad \times s\left(a_{1}, \ldots, a_{m}+\left\lceil\frac{x+z}{2}\right\rceil, c_{1}, \ldots, c_{k}+\left\lfloor\frac{x+z}{2}\right\rfloor+b_{n}, b_{n-1}, \ldots, b_{1}\right) \\
& \quad \times s\left(y+b-\min (a, b), a_{1}, \ldots, a_{m},\left\lceil\frac{x+z}{2} \mid+c_{1}, c_{2},\right.\right. \\
& \left.\quad \ldots, c_{k},\left\lfloor\frac{x+z}{2}\right\rfloor, b_{n}, \ldots, b_{1}, y+a-\min (a, b)+2\right) \\
& \quad \times \frac{\mathrm{H}\left(c+\frac{x+z}{2}\right)}{\mathrm{H}(c) \mathrm{H}\left(\frac{x+z}{2}\right)} \frac{\mathrm{H}\left(\max (a, b)+y+\frac{x+z}{2}+1\right)}{\mathrm{H}\left(\max (a, b)+c+y+\frac{x+z}{2}+1\right)} \\
& \quad \times \frac{\mathrm{H}(\max (a, b)+y+z) \mathrm{H}(\max (a, b)+c+y+z+2)}{\mathrm{H}\left(o_{a}+o_{b}+o_{c}+z\right) \mathrm{H}\left(|a-b|+e_{a}+e_{b}+e_{c}+2 y+z+2\right)} \\
& \quad \times \frac{\mathrm{H}\left(o_{a}+o_{b}+o_{c}\right) \mathrm{H}\left(a-b \mid+e_{a}+e_{b}+e_{c}+2 y+2\right)}{\mathrm{H}(\max (a, b)+y) \mathrm{H}(\max (a, b)+y+2)},
\end{aligned}
$$

where $\Theta_{x, y, z}^{\prime}(m)$ is defined as in $\left(T^{\prime}-3\right)$.

Theorem 34. Assume that $\boldsymbol{a}=\left(a_{1}, a_{2}, \ldots, a_{m}\right), \boldsymbol{b}=\left(b_{1}, b_{2}, \ldots, b_{n}\right), \boldsymbol{c}=\left(c_{1}, c_{2}, \ldots, c_{k}\right)$ are three sequences of nonnegative integers ( $m, n, k$ are all even) and that $x, y, z$ are three 
integers, such that $x \geqslant 0, y \geqslant \max (a-b,-2), z \geqslant 0$, and $x$ and $z$ have different parities. Then

$$
\begin{aligned}
& \mathrm{M}\left(\bar{F}_{x, y, z}^{(6)}(\boldsymbol{a} ; \boldsymbol{c} ; \boldsymbol{b})\right)=\Lambda_{x, 2 y+z+2 \max (a, b)+2, z}^{\prime}(c) \\
& \quad \times s\left(a_{1}, \ldots, a_{m}+\left\lceil\frac{x+z}{2}\right]+1, c_{1}, \ldots, c_{k}+\left\lfloor\frac{x+z}{2}\right\rfloor+b_{n}-1, b_{n-1}, \ldots, b_{1}\right) \\
& \quad \times s\left(y+b-\min (a, b), a_{1}, \ldots, a_{m},\left\lceil\frac{x+z}{2} \mid+c_{1}+1, c_{2},\right.\right. \\
& \quad \ldots, c_{k},\left\lfloor\frac{x+z}{2} \mid-1, b_{n}, \ldots, b_{1}, y+a-\min (a, b)+2\right) \\
& \quad \times \frac{\mathrm{H}\left(c+\frac{x+z}{2}-1\right)}{\mathrm{H}(c) \mathrm{H}\left(\frac{x+z}{2}-1\right)} \frac{\mathrm{H}\left(\max (a, b)+y+\frac{x+z}{2}+1\right)}{\mathrm{H}\left(\max (a, b)+c+y+\frac{x+z}{2}+1\right)} \\
& \quad \times \frac{\mathrm{H}(\max (a, b)+y+z) \mathrm{H}(\max (a, b)+c+y+z+2)}{\mathrm{H}\left(o_{a}+o_{b}+o_{c}+z\right) \mathrm{H}\left(|a-b|+e_{a}+e_{b}+e_{c}+2 y+z+2\right)} \\
& \quad \times \frac{\mathrm{H}\left(o_{a}+o_{b}+o_{c}\right) \mathrm{H}\left(|a-b|+e_{a}+e_{b}+e_{c}+2 y+2\right)}{\mathrm{H}(\max (a, b)+y) \mathrm{H}(\max (a, b)+y+2)}
\end{aligned}
$$

where $\Lambda_{x, y, z}^{\prime}(m)$ is defined as in $\left(T^{\prime}-4\right)$.

\subsection{The $\bar{G}^{(i)}$-type regions}

Our regions in this section are defined by applying the pushing procedure in the definition of the $\bar{E}^{(i)}$-type regions to the auxiliary hexagon as in the case of the $G^{(i)}$-type regions. In particular, we are assuming that $x$ and $z$ are two nonnegative integers having the same parity and that $y$ is an integer with domain defined particularly in the theorems below. For $i=2,5$, we start with an auxiliary hexagon of side-lengths $x, z+1, z, x, z+1, z$, for $i=3,4$, we have the auxiliary hexagon of side-lengths $x, z+3, z, x, z+3, z$. Next, we perform the 2-stage pushing to all six sides of the auxiliary hexagons above in the same way as in the definition of the $\bar{E}^{(i)}$-type regions to obtain the base hexagon $H$. Finally, the three ferns are removed from the base hexagon $H$, such that the leftmost of the middle fern is at the off-central positions $i$ as indicated in Figure 4(c). There are no counterparts for $i=1,6,7,8$. Denote by $\bar{G}_{x, y, z}^{(i)}(\mathbf{a} ; \mathbf{c} ; \mathbf{b})$ the resulting regions, for $i=2,3,4,5$.

Remark 35. In the definition of the $\bar{G}^{(2)}$-type regions, if we remove the three ferns such that the root of the middle one is $1 / 2$-unit to the northwest of the center of the auxiliary hexagon $H_{0}$, then we obtain the region $Q_{x, y, z}^{\nwarrow}(\mathbf{a} ; \mathbf{c} ; \mathbf{b})$ in Theorem 2.7 of [31]. This way, the $\bar{G}^{(i)}$-type regions can be viewed as off-central counterparts of the $Q^{\nwarrow}$-type regions.

Theorem 36. Assume that $\boldsymbol{a}=\left(a_{1}, a_{2}, \ldots, a_{m}\right), \boldsymbol{b}=\left(b_{1}, b_{2}, \ldots, b_{n}\right), \boldsymbol{c}=\left(c_{1}, c_{2}, \ldots, c_{k}\right)$ are three sequences of nonnegative integers ( $m, n, k$ are all even) and that $x, y, z$ are three 


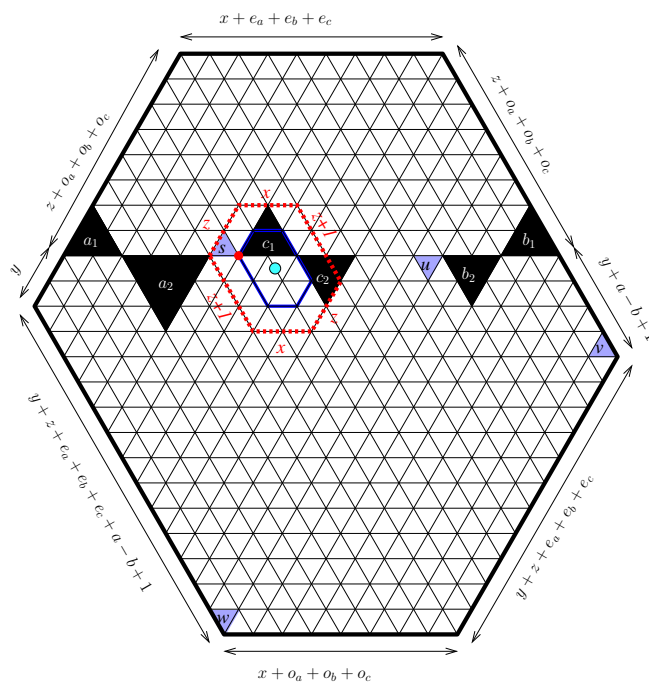

(a)

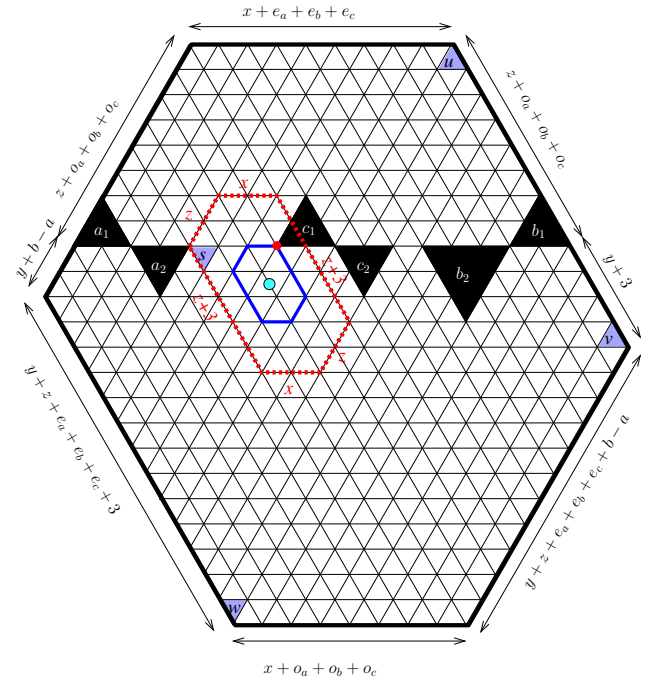

(c)

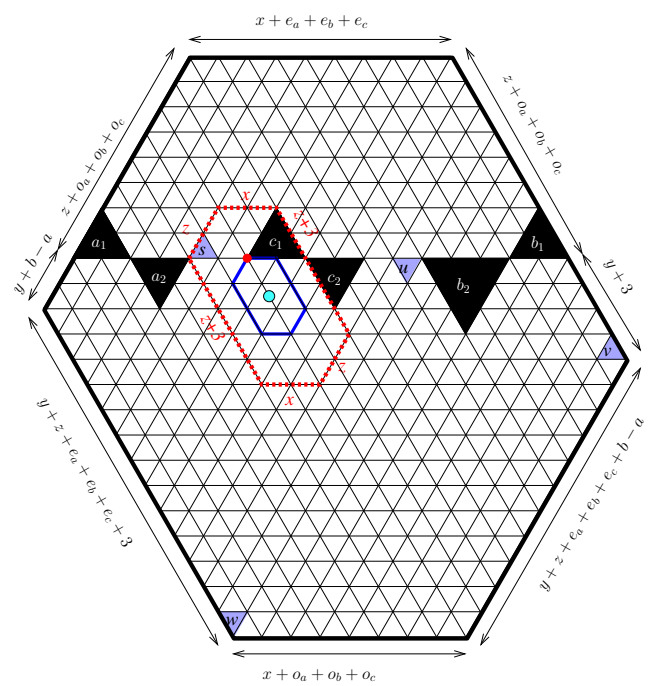

(b)

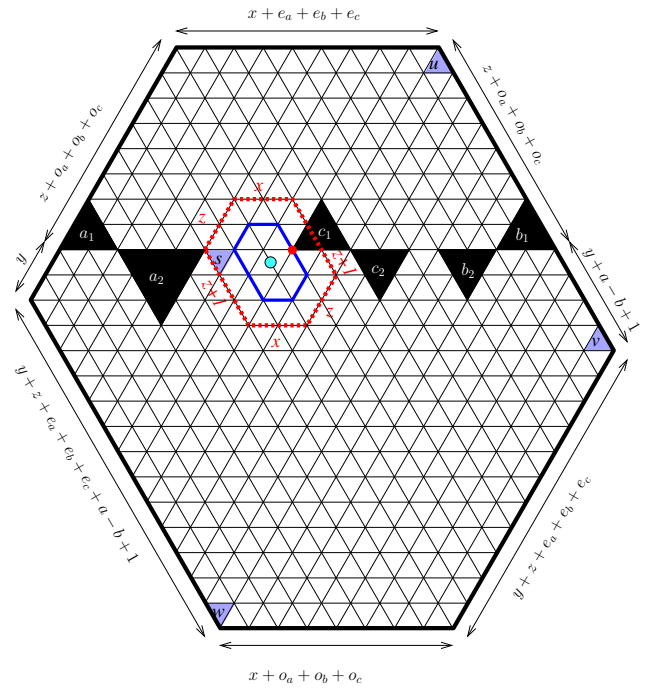

(d)

Figure 13: Four $\bar{G}^{(i)}$-type regions: (a) $\bar{G}_{2,2,2}^{(2)}(2,3 ; 2,2 ; 2,2)$, (b) $\bar{G}_{2,1,2}^{(3)}(2,2 ; 2,2 ; 2,3)$, (c) $\bar{G}_{2,1,2}^{(4)}(2,2 ; 2,2 ; 2,3)$, and (d) $\bar{G}_{2,2,2}^{(5)}(2,3 ; 2,2 ; 2,2)$.

integers, such that $x \geqslant 0, y \geqslant \max (a-b,-1), z \geqslant 0$, and $x$ and $z$ have the same parity. Then

$$
\begin{aligned}
& \mathrm{M}\left(\bar{G}_{x, y, z}^{(2)}(\boldsymbol{a} ; \boldsymbol{c} ; \boldsymbol{b})\right)=\Lambda_{2 y+z+2 \max (a, b)+1, z, x}^{\prime}(c) \\
& \quad \times s\left(a_{1}, \ldots, a_{m}+\frac{x+z}{2}-1, c_{1}, \ldots, c_{k}+\frac{x+z}{2}+b_{n}+1, b_{n-1}, \ldots, b_{1}\right) \\
& \quad \times s\left(y+b-\min (a, b), a_{1}, \ldots, a_{m}, \frac{x+z}{2}+c_{1}-1, c_{2},\right.
\end{aligned}
$$




$$
\begin{aligned}
& \left.\qquad \ldots, c_{k}, \frac{x+z}{2}+1, b_{n}, \ldots, b_{1}, y+a-\min (a, b)+1\right) \\
& \times \frac{\mathrm{H}\left(c+\frac{x+z}{2}-1\right)}{\mathrm{H}(c) \mathrm{H}\left(\frac{x+z}{2}-1\right)} \frac{\mathrm{H}\left(\max (a, b)+y+\frac{x+z}{2}-1\right)}{\mathrm{H}\left(\max (a, b)+c+y+\frac{x+z}{2}-1\right)} \\
& \times \frac{\mathrm{H}(\max (a, b)+y+z+1) \mathrm{H}(\max (a, b)+c+y+z)}{\mathrm{H}\left(o_{a}+o_{b}+o_{c}+z\right) \mathrm{H}\left(|a-b|+e_{a}+e_{b}+e_{c}+2 y+z+1\right)} \\
& \times \frac{\mathrm{H}\left(o_{a}+o_{b}+o_{c}\right) \mathrm{H}\left(|a-b|+e_{a}+e_{b}+e_{c}+2 y\right)}{\mathrm{H}(\max (a, b)+y) \mathrm{H}(\max (a, b)+y+1)},
\end{aligned}
$$

where $\Lambda_{x, y, z}^{\prime}(m)$ is defined as in $\left(T^{\prime}-4\right)$.

Theorem 37. Assume that $\boldsymbol{a}=\left(a_{1}, a_{2}, \ldots, a_{m}\right), \boldsymbol{b}=\left(b_{1}, b_{2}, \ldots, b_{n}\right), \boldsymbol{c}=\left(c_{1}, c_{2}, \ldots, c_{k}\right)$ are three sequences of nonnegative integers ( $m, n, k$ are all even) and that $x, y, z$ are three integers, such that $x \geqslant 0, y \geqslant \max (a-b,-3), z \geqslant 0$, and $x$ and $z$ have the same parity. Then

$$
\begin{aligned}
\mathrm{M} & \left(\bar{G}_{x, y, z}^{(3)}(\boldsymbol{a} ; \boldsymbol{c} ; \boldsymbol{b})\right)=\Psi_{2 y+z+2 \max (a, b)+3, z, x}^{\prime}(c) \\
& \times s\left(a_{1}, \ldots, a_{m}+\frac{x+z}{2}, c_{1}, \ldots, c_{k}+\frac{x+z}{2}+b_{n}, b_{n-1}, \ldots, b_{1}\right) \\
& \times s\left(y+b-\min (a, b), a_{1}, \ldots, a_{m}, \frac{x+z}{2}+c_{1}, c_{2},\right. \\
& \left.\ldots, c_{k}, \frac{x+z}{2}, b_{n}, \ldots, b_{1}, y+a-\min (a, b)+3\right) \\
& \times \frac{\mathrm{H}\left(c+\frac{x+z}{2}\right)}{\mathrm{H}(c) \mathrm{H}\left(\frac{x+z}{2}\right)} \frac{\mathrm{H}\left(\max (a, b)+y+\frac{x+z}{2}\right)}{\mathrm{H}\left(\max (a, b)+c+y+\frac{x+z}{2}\right)} \\
& \times \frac{\mathrm{H}(\max (a, b)+y+z+3) \mathrm{H}(\max (a, b)+c+y+z)}{\mathrm{H}\left(o_{a}+o_{b}+o_{c}+z\right) \mathrm{H}\left(|a-b|+e_{a}+e_{b}+e_{c}+2 y+z+3\right)} \\
& \times \frac{\mathrm{H}\left(o_{a}+o_{b}+o_{c}\right) \mathrm{H}\left(|a-b|+e_{a}+e_{b}+e_{c}+2 y+3\right)}{\mathrm{H}(\max (a, b)+y) \mathrm{H}(\max (a, b)+y+3)},
\end{aligned}
$$

where $\Psi_{x, y, z}^{\prime}(m)$ is the formula in (T'-2).

Theorem 38. Assume that $\boldsymbol{a}=\left(a_{1}, a_{2}, \ldots, a_{m}\right), \boldsymbol{b}=\left(b_{1}, b_{2}, \ldots, b_{n}\right), \boldsymbol{c}=\left(c_{1}, c_{2}, \ldots, c_{k}\right)$ are three sequences of nonnegative integers ( $m, n, k$ are all even) and that $x, y, z$ are three integers, such that $x \geqslant 0, y \geqslant \max (a-b,-3), z \geqslant 0$, and $x$ and $z$ have the same parity. Then

$$
\begin{aligned}
& \mathrm{M}\left(\bar{G}_{x, y, z}^{(4)}(\boldsymbol{a} ; \boldsymbol{c} ; \boldsymbol{b})\right)=\Lambda_{z, 2 y+z+2 \max (a, b)+3, x}(c) \\
& \quad \times s\left(a_{1}, \ldots, a_{m}+\frac{x+z}{2}+1, c_{1}, \ldots, c_{k}+\frac{x+z}{2}+b_{n}, b_{n-1}-1, \ldots, b_{1}\right) \\
& \quad \times s\left(y+b-\min (a, b), a_{1}, \ldots, a_{m}, \frac{x+z}{2}+c_{1}+1, c_{2},\right.
\end{aligned}
$$




$$
\begin{aligned}
& \left.\quad \ldots, c_{k}, \frac{x+z}{2}-1, b_{n}, \ldots, b_{1}, y+a-\min (a, b)+3\right) \\
& \times \frac{\mathrm{H}\left(c+\frac{x+z}{2}+1\right)}{\mathrm{H}(c) \mathrm{H}\left(\frac{x+z}{2}+1\right)} \frac{\mathrm{H}\left(\max (a, b)+y+\frac{x+z}{2}+1\right)}{\mathrm{H}\left(\max (a, b)+c+y+\frac{x+z}{2}+1\right)} \\
& \times \frac{\mathrm{H}(\max (a, b)+y+z+3) \mathrm{H}(\max (a, b)+c+y+z)}{\left.\mathrm{H}\left(o_{a}+o_{b}+o_{c}+z\right)\right) \mathrm{H}\left(|a-b|+e_{a}+e_{b}+e_{c}+2 y+z+3\right)} \\
& \times \frac{\mathrm{H}\left(o_{a}+o_{b}+o_{c}\right) \mathrm{H}\left(|a-b|+e_{a}+e_{b}+e_{c}+2 y+3\right)}{\mathrm{H}(\max (a, b)+y) \mathrm{H}(\max (a, b)+y+3)},
\end{aligned}
$$

where $\Lambda_{x, y, z}(m)$ is defined as in $(T-4)$.

Theorem 39. Assume that $\boldsymbol{a}=\left(a_{1}, a_{2}, \ldots, a_{m}\right), \boldsymbol{b}=\left(b_{1}, b_{2}, \ldots, b_{n}\right), \boldsymbol{c}=\left(c_{1}, c_{2}, \ldots, c_{k}\right)$ are three sequences of nonnegative integers ( $m, n, k$ are all even) and that $x, y, z$ are three integers, such that $x \geqslant 0, y \geqslant \max (a-b,-1), z \geqslant 0$, and $x$ and $z$ have the same parity. Then

$$
\begin{aligned}
& \mathrm{M}\left(\bar{G}_{x, y, z}^{(5)}(\boldsymbol{a} ; \boldsymbol{c} ; \boldsymbol{b})\right)=\Theta_{2 y+z+2 \max (a, b)+1, z, x}(c) \\
& \quad \times s\left(a_{1}, \ldots, a_{m}+\frac{x+z}{2}+1, c_{1}, \ldots, c_{k}+\frac{x+z}{2}+b_{n}, b_{n-1}-1, \ldots, b_{1}\right) \\
& \quad \times s\left(y+b-\min (a, b), a_{1}, \ldots, a_{m}, \frac{x+z}{2}+c_{1}+1, c_{2},\right. \\
& \left.\ldots, c_{k}, \frac{x+z}{2}-1, b_{n}, \ldots, b_{1}, y+a-\min (a, b)+1\right) \\
& \quad \times \frac{\mathrm{H}\left(c+\frac{x+z}{2}-1\right)}{\mathrm{H}(c) \mathrm{H}\left(\frac{x+z}{2}-1\right)} \frac{\mathrm{H}\left(\max (a, b)+y+\frac{x+z}{2}\right)}{\mathrm{H}\left(\max (a, b)+c+y+\frac{x+z}{2}\right)} \\
& \quad \times \frac{\mathrm{H}(\max (a, b)+y+z) \mathrm{H}(\max (a, b)+c+y+z+1)}{\left.\mathrm{H}\left(o_{a}+o_{b}+o_{c}+z\right)\right) \mathrm{H}\left(|a-b|+e_{a}+e_{b}+e_{c}+2 y+z+1\right)} \\
& \quad \times \frac{\mathrm{H}\left(o_{a}+o_{b}+o_{c}\right) \mathrm{H}\left(|a-b|+e_{a}+e_{b}+e_{c}+2 y+1\right)}{\mathrm{H}(\max (a, b)+y) \mathrm{H}(\max (a, b)+y+1)},
\end{aligned}
$$

where $\Theta_{x, y, z}(m)$ is defined as in (T-3).

\subsection{The $\bar{K}^{(i)}$-type regions}

We now define the final group of the off-central regions. We also start with an auxiliary hexagon $H_{0}$ whose side-lengths are $x, z+1, z, x, z+1, z$ for $i=5,8$, and are $x, z+3, z, x, z+$ $3, z$, for $i=6,7$ ( $x$ and $z$ have different parities in this case). After pushing the sides of the auxiliary hexagon $H_{0}$ in the same way as in the definition of the $\bar{E}^{(i)}$-type regions, we remove three ferns such that the root of the middle fern is at the off-central positions $i$ as shown in Figure $4(\mathrm{~d})$. There are no counterparts for the case $i=1,2,3,4$ here. Let $\bar{K}^{(i)}$ denote the newly defined region, $i=5,6,7,8$. 


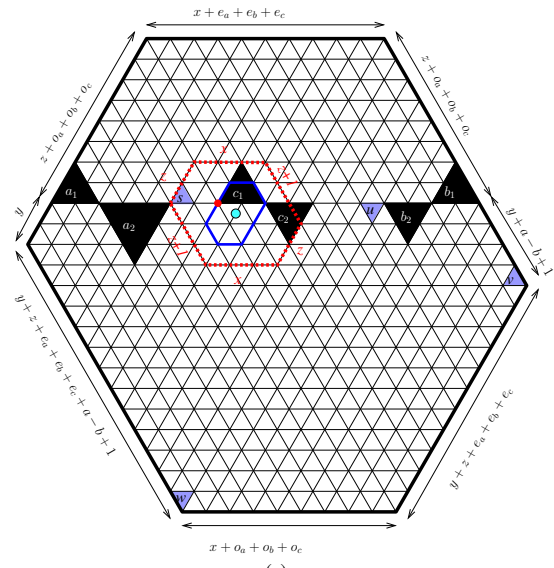

(a)

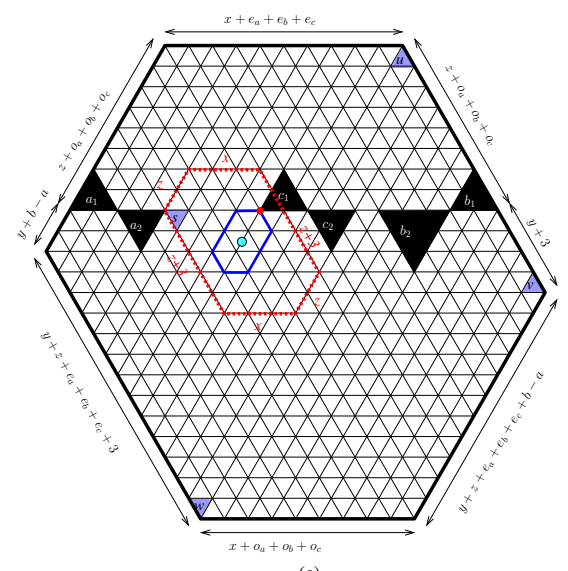

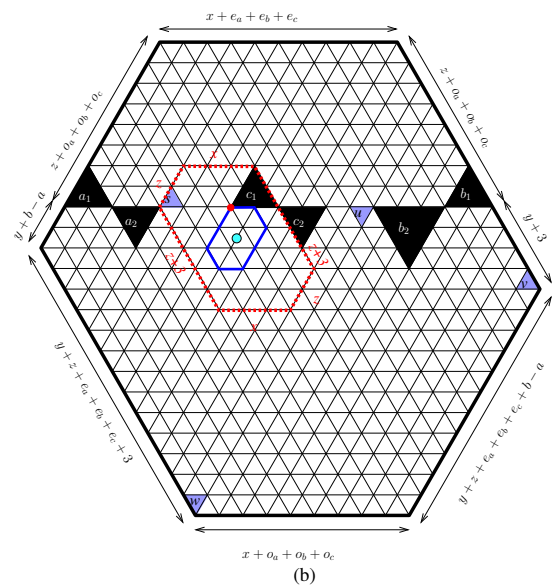

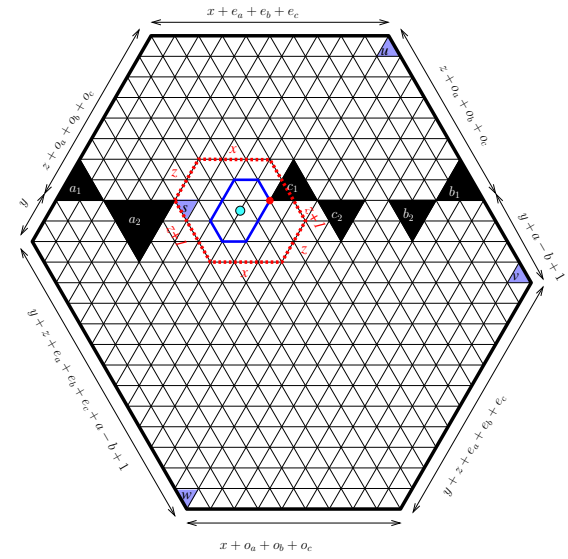

(d)

Figure 14: Four $\bar{K}^{(i)}$-type regions: (a) $\bar{K}_{3,2,2}^{(5)}(2,3 ; 2,2 ; 2,2)$, (b) $\bar{K}_{3,2,2}^{(6)}(2,2 ; 2,2 ; 2,3)$, (c) $\bar{K}_{3,2,2}^{(7)}(2,2 ; 2,2 ; 2,3)$, and (d) $\bar{K}_{3,2,2}^{(8)}(2,3 ; 2,2 ; 2,2)$.

Remark 40. In the definition of the $\bar{K}^{(5)}$-type regions, if we remove the three ferns such that the root of the middle one is $1 / 2$-unit to the southwest of the center of the auxiliary hexagon $H_{0}$, then we obtain exactly the region $Q_{x, y, z}^{\swarrow}(\mathbf{a} ; \mathbf{c} ; \mathbf{b})$ in Theorem 2.8 of [31]. One could view the $\bar{K}^{(i)}$-type regions as off-central counterparts of the $Q^{\swarrow}$-type regions.

Theorem 41. Assume that $\boldsymbol{a}=\left(a_{1}, a_{2}, \ldots, a_{m}\right), \boldsymbol{b}=\left(b_{1}, b_{2}, \ldots, b_{n}\right), \boldsymbol{c}=\left(c_{1}, c_{2}, \ldots, c_{k}\right)$ are three sequences of nonnegative integers ( $m, n, k$ are all even) and that $x, y, z$ are three integers, such that $x \geqslant 0, y \geqslant \max (a-b,-1), z \geqslant 0$, and $x$ and $z$ have different parities. Then

$$
\begin{aligned}
& \mathrm{M}\left(\bar{K}_{x, y, z}^{(5)}(\boldsymbol{a} ; \boldsymbol{c} ; \boldsymbol{b})\right)=\Theta_{z, x, 2 y+z+2 \max (a, b)+1}(c) \\
& \quad \times s\left(a_{1}, \ldots, a_{m}+\left\lfloor\frac{x+z}{2}\right\rfloor, c_{1}, \ldots, c_{k}+\left\lceil\frac{x+z}{2}\right\rceil+b_{n}, b_{n-1}, \ldots, b_{1}\right) \\
& \quad \times s\left(y+b-\min (a, b), a_{1}, \ldots, a_{m},\left\lfloor\frac{x+z}{2}\right\rfloor+c_{1}, c_{2},\right.
\end{aligned}
$$




$$
\begin{aligned}
\left.\ldots, c_{k},\left\lceil\frac{x+z}{2}\right\rceil, b_{n}, \ldots, b_{1}, y+a-\min (a, b)+1\right) \\
\times \frac{\mathrm{H}\left(c+\left\lceil\frac{x+z}{2}\right\rceil\right)}{\mathrm{H}(c) \mathrm{H}\left(\left\lceil\frac{x+z}{2}\right\rceil\right)} \frac{\mathrm{H}\left(\max (a, b)+y+\left\lceil\frac{x+z}{2}\right\rceil+1\right)}{\mathrm{H}\left(\max (a, b)+c+y+\left\lceil\frac{x+z}{2}\right\rceil+1\right.} \\
\times \frac{\mathrm{H}(\max (a, b)+y+z) \mathrm{H}(\max (a, b)+c+y+z+1)}{\left.\mathrm{H}\left(o_{a}+o_{b}+o_{c}+z\right)\right) \mathrm{H}\left(|a-b|+e_{a}+e_{b}+e_{c}+2 y+z+1\right)} \\
\times \frac{\mathrm{H}\left(o_{a}+o_{b}+o_{c}\right) \mathrm{H}\left(|a-b|+e_{a}+e_{b}+e_{c}+2 y+1\right)}{\mathrm{H}(\max (a, b)+y) \mathrm{H}(\max (a, b)+y+1)},
\end{aligned}
$$

where $\Theta_{x, y, z}(m)$ is defined as in (T-3).

Theorem 42. Assume that $\boldsymbol{a}=\left(a_{1}, a_{2}, \ldots, a_{m}\right), \boldsymbol{b}=\left(b_{1}, b_{2}, \ldots, b_{n}\right), \boldsymbol{c}=\left(c_{1}, c_{2}, \ldots, c_{k}\right)$ are three sequences of nonnegative integers ( $m, n, k$ are all even) and that $x, y, z$ are three integers, such that $x \geqslant 0, y \geqslant \max (a-b,-3), z \geqslant 0$, and $x$ and $z$ have different parities. Then

$$
\begin{aligned}
& \mathrm{M}\left(\bar{K}_{x, y, z}^{(6)}(\boldsymbol{a} ; \boldsymbol{c} ; \boldsymbol{b})\right)=\Lambda_{z, x, 2 y+z+2 \max (a, b)+3}(c) \\
& \quad \times s\left(a_{1}, \ldots, a_{m}+\left\lceil\frac{x+z}{2}\right\rceil, c_{1}, \ldots, c_{k}+\left\lfloor\frac{x+z}{2}\right\rfloor+b_{n}, b_{n-1}, \ldots, b_{1}\right) \\
& \quad \times s\left(y+b-\min (a, b), a_{1}, \ldots, a_{m},\left\lceil\frac{x+z}{2}\right\rceil+c_{1}, c_{2},\right. \\
& \left.\quad \ldots, c_{k},\left\lfloor\frac{x+z}{2}\right\rfloor, b_{n}, \ldots, b_{1}, y+a-\min (a, b)+3\right) \\
& \quad \times \frac{\mathrm{H}\left(c+\left\lfloor\frac{x+z}{2}\right\rfloor\right)}{\mathrm{H}(c) \mathrm{H}\left(\left\lfloor\frac{x+z}{2}\right\rfloor\right)} \frac{\mathrm{H}\left(\max (a, b)+y+\left\lceil\frac{x+z}{2}\right\rceil+2\right)}{\mathrm{H}\left(\max (a, b)+c+y+\left\lceil\frac{x+z}{2}\right\rceil+2\right)} \\
& \quad \times \frac{\mathrm{H}(\max (a, b)+y+z) \mathrm{H}(\max (a, b)+c+y+z+3)}{\left.\mathrm{H}\left(o_{a}+o_{b}+o_{c}+z\right)\right) \mathrm{H}\left(|a-b|+e_{a}+e_{b}+e_{c}+2 y+z+3\right)} \\
& \quad \times \frac{\mathrm{H}\left(o_{a}+o_{b}+o_{c}\right) \mathrm{H}\left(|a-b|+e_{a}+e_{b}+e_{c}+2 y+3\right)}{\mathrm{H}(\max (a, b)+y) \mathrm{H}(\max (a, b)+y+3)},
\end{aligned}
$$

where $\Lambda_{x, y, z}(m)$ is defined as in $(T-4)$.

Theorem 43. Assume that $\boldsymbol{a}=\left(a_{1}, a_{2}, \ldots, a_{m}\right), \boldsymbol{b}=\left(b_{1}, b_{2}, \ldots, b_{n}\right), \boldsymbol{c}=\left(c_{1}, c_{2}, \ldots, c_{k}\right)$ are three sequences of nonnegative integers ( $m, n, k$ are all even) and that $x, y, z$ are three integers, such that $x \geqslant 0, y \geqslant \max (a-b,-3), z \geqslant 0$, and $x$ and $z$ have different parities. Then

$$
\begin{aligned}
& \mathrm{M}\left(\bar{K}_{x, y, z}^{(7)}(\boldsymbol{a} ; \boldsymbol{c} ; \boldsymbol{b})\right)=\Psi_{z, x, 2 y+z+2 \max (a, b)+3}(c) \\
& \quad \times s\left(a_{1}, \ldots, a_{m}+\left\lceil\frac{x+z}{2}\right\rceil+1, c_{1}, \ldots, c_{k}+\left\lfloor\frac{x+z}{2}\right\rfloor-1+b_{n}, b_{n-1}, \ldots, b_{1}\right) \\
& \quad \times s\left(y+b-\min (a, b), a_{1}, \ldots, a_{m},\left\lceil\frac{x+z}{2}\right\rceil+c_{1}+1, c_{2},\right.
\end{aligned}
$$




$$
\begin{aligned}
\left.\ldots, \ldots, c_{k},\left\lfloor\frac{x+z}{2}\right\rfloor-1, b_{n}, \ldots, b_{1}, y+a-\min (a, b)+3\right) \\
\times \frac{\mathrm{H}\left(c+\left\lfloor\frac{x+z}{2}\right\rfloor-1\right)}{\mathrm{H}(c) \mathrm{H}\left(\left\lfloor\frac{x+z}{2}\right\rfloor-1\right)} \frac{\mathrm{H}\left(\max (a, b)+y+\left\lceil\frac{x+z}{2}\right\rceil+1\right)}{\mathrm{H}\left(\max (a, b)+c+y+\left\lceil\frac{x+z}{2}\right\rceil+1\right)} \\
\times \frac{\mathrm{H}(\max (a, b)+y+z) \mathrm{H}(\max (a, b)+c+y+z+3)}{\left.\mathrm{H}\left(o_{a}+o_{b}+o_{c}+z\right)\right) \mathrm{H}\left(|a-b|+e_{a}+e_{b}+e_{c}+2 y+z+3\right)} \\
\times \frac{\mathrm{H}\left(o_{a}+o_{b}+o_{c}\right) \mathrm{H}\left(|a-b|+e_{a}+e_{b}+e_{c}+2 y+3\right)}{\mathrm{H}(\max (a, b)+y) \mathrm{H}(\max (a, b)+y+3)},
\end{aligned}
$$

where $\Psi_{x, y, z}(m)$ is defined as in (T-2).

Theorem 44. Assume that $\boldsymbol{a}=\left(a_{1}, a_{2}, \ldots, a_{m}\right), \boldsymbol{b}=\left(b_{1}, b_{2}, \ldots, b_{n}\right), \boldsymbol{c}=\left(c_{1}, c_{2}, \ldots, c_{k}\right)$ are three sequences of nonnegative integers $(m, n, k$ are all even)and that $x, y, z$ are three integers, such that $x \geqslant 0, y \geqslant \max (a-b,-1), z \geqslant 0$, and $x$ and $z$ have the same parity. Then

$$
\begin{aligned}
\mathrm{M} & \left.\bar{K}_{x, y, z}^{(8)}(\boldsymbol{a} ; \boldsymbol{c} ; \boldsymbol{b})\right)=\Lambda_{2 y+z+2 \max (a, b)+1, x, z}^{\prime}(c) \\
& \times s\left(a_{1}, \ldots, a_{m}+\left\lceil\frac{x+z}{2}\right\rceil+1, c_{1}, \ldots, c_{k}+\left\lfloor\frac{x+z}{2}\right\rfloor-1+b_{n}, b_{n-1}, \ldots, b_{1}\right) \\
& \times s\left(y+b-\min (a, b), a_{1}, \ldots, a_{m},\left\lceil\frac{x+z}{2} \mid+c_{1}+1, c_{2},\right.\right. \\
& \left.\ldots, c_{k},\left\lfloor\frac{x+z}{2}\right\rfloor-1, b_{n}, \ldots, b_{1}, y+a-\min (a, b)+1\right) \\
& \times \frac{\mathrm{H}\left(c+\left\lfloor\frac{x+z}{2}\right\rfloor-1\right)}{\mathrm{H}(c) \mathrm{H}\left(\left\lfloor\frac{x+z}{2}\right\rfloor-1\right)} \frac{\mathrm{H}\left(\max (a, b)+y+\left\lceil\frac{x+z}{2}\right\rceil\right)}{\mathrm{H}\left(\max (a, b)+c+y+\left\lceil\frac{x+z}{2}\right\rceil\right)} \\
& \times \frac{\mathrm{H}(\max (a, b)+y+z) \mathrm{H}(\max (a, b)+c+y+z+1)}{\left.\mathrm{H}\left(o_{a}+o_{b}+o_{c}+z\right)\right) \mathrm{H}\left(|a-b|+e_{a}+e_{b}+e_{c}+2 y+z+1\right)} \\
& \times \frac{\mathrm{H}\left(o_{a}+o_{b}+o_{c}\right) \mathrm{H}\left(|a-b|+e_{a}+e_{b}+e_{c}+2 y+1\right)}{\mathrm{H}(\max (a, b)+y) \mathrm{H}(\max (a, b)+y+1)},
\end{aligned}
$$

where $\Lambda_{x, y, z}^{\prime}(m)$ is defined as in $\left(T^{\prime}-4\right)$.

\section{Proofs of the main theorems}

\subsection{Organization of the proof}

Recall that our eight families of off-central regions, $E^{(i)}, F^{(i)}, G^{(i)}, K^{(i)}, \bar{E}^{(i)}, \bar{F}^{(i)}, \bar{G}^{(i)}$, and $\bar{K}^{(i)}$, are all obtained from a particular base hexagon $H$ by removing three ferns along a common lattice line $l$. We call the perimeter of the base hexagon the quasi-perimeter of the regions, denoted by $p$ in the rest of the proof.

Lemma 45. We always have for all regions in the eight families

$$
p \geqslant 2 x+4 z .
$$


Proof. If we start with an auxiliary hexagon $H_{0}$ of side-lengths $x, z+j, z, x, z+j, z$ (for $j=0,1,2,3)$ in the definition of our region, then the quasi perimeter is always

$$
p=2 x+4 y+4 z+3 a+3 b+2|a-b|+2 j .
$$

In this case, we always have $y \geqslant \max (-|a-b|,-j)$. It means that

$$
4 y+2|a-b|+2 j \geqslant 0,
$$

so $p \geqslant 2 x+4 z+3 a+3 b \geqslant 2 x+4 z$.

We aim to prove all 30 tiling formulas in Theorems $4-44$ at once by induction on $h:=p+x+z$, where $p$ is the quasi-perimeter of the region. Our proof is organized as follows. In Section 3.2, we quote the particular versions of Kuo condensation that will be employed in our proofs. Next, in Sections 3.3-3.10, we will carefully present 66 recurrences for our eight families of regions obtained by applying Kuo condensation. Each family of regions will have more than one recurrence, depending on whether $a>b, a=b$, or $a<b$. We want to emphasize that, due to the difference in the structures of our regions, the universal recurrence does not seem to exist. In Section 3.11, we investigate the two extremal cases of our proof. Section 3.12 is devoted to the main arguments of the inductive proof.

\subsection{Kuo condensation and other preliminary results}

A forced lozenge in a region $R$ on the triangular lattice is a lozenge contained in any tiling of $R$. Removal of forced lozenges does not change the tiling number of a region.

A region on the triangular lattice is balanced if it has the same number of up- and down-pointing unit triangles (this is a necessary condition that a region admits a tiling). The following useful lemma allows us to decompose a large region into several smaller ones.

Lemma 46 (Region-splitting Lemma $[26,27])$. Let $R$ be a balanced region on the triangular lattice. Assume that a blanched sub-region $Q$ of $R$ satisfies the condition that only one type of unit triangles runs along each side of the border between $Q$ and $R-Q$. Then $\mathrm{M}(R)=\mathrm{M}(Q) \cdot \mathrm{M}(R-Q)$.

Let $G$ be a finite graph without loops; however, the multiple-edges are allowed. A perfect matching of $G$ is a collection of disjoint edges covering all vertices of $G$. The (planar) dual graph of a region $R$ on the triangular lattice is the graph whose vertices are unit triangles in $R$ and whose edges connect precisely those two unit triangles sharing an edge. We can identify the tilings of a region with the perfect matchings of its dual graph. In this point of view, we use the notation $\mathrm{M}(G)$ for the number of perfect matchings of graph $G$.

The following two theorems of Kuo are the key to our proofs in this paper. 
Theorem 47 (Theorem $5.1[21])$. Let $G=\left(V_{1}, V_{2}, E\right)$ be a bipartite planar graph in which $\left|V_{1}\right|=\left|V_{2}\right|$. Assume that $u, v, w, s$ are four vertices appearing in a cyclic order on a face of $G$ with $u, w \in V_{1}$ and $v, s \in V_{2}$. Then

$\mathrm{M}(G) \mathrm{M}(G-\{u, v, w, s\})=\mathrm{M}(G-\{u, v\}) \mathrm{M}(G-\{w, s\})+\mathrm{M}(G-\{u, s\}) \mathrm{M}(G-\{v, w\})$.

Theorem 48 (Theorem $5.2[21])$. Let $G=\left(V_{1}, V_{2}, E\right)$ be a bipartite planar graph in which $\left|V_{1}\right|=\left|V_{2}\right|$. Assume that $u, v, w, s$ are four vertices appearing in a cyclic order on a face of $G$ with $u, v \in V_{1}$ and $w, s \in V_{2}$. Then

$\mathrm{M}(G-\{u, s\}) \mathrm{M}(G-\{v, w\})=\mathrm{M}(G) \mathrm{M}(G-\{u, v, w, s\})+\mathrm{M}(G-\{u, w\}) \mathrm{M}(G-\{v, s\})$.

Theorems 47 and 48 are usually mentioned as two variants of Kuo condensation. Kuo condensation (or graphical condensation as called in [21]) can be considered as a combinatorial interpretation of the well-known Dodgson condensation in linear algebra (which is based on the Jacobi-Desnanot identity, see, e.g., [1], [13] and [36], pp. 136148, and [49] for a bijective proof). The Dodgson condensation was named after Charles Lutwidge Dodgson (1832-1898), better known by his pen name Lewis Carroll, an English writer, mathematician, and photographer.

Eric H. Kuo introduced Kuo condensation in his 2004 paper [21] with four different versions, two of them are Theorems 47 and 48 stated above. The preliminary version of Kuo condensation (when the four vertices $u, v, w, s$ in Theorems 47 and and 48 form a 4-cycle in the graph $G$ ) was initially conjectured by Alexandru Ionescu in the context of Aztec diamond graphs and was proved by Propp in 1993 (see [39]). Kuo condensation has become a powerful tool in the enumeration of tilings with many applications. We refer the reader to, e.g., $[5,14,18,22,44,47,48]$ for various aspects and generalizations of Kuo condensation, and e.g., $[8,10,11,17,24-30,32-34,41,42]$ for recent applications of the method.

\subsection{Recurrences for $E^{(i)}$-type regions}

We apply Kuo condensation in Theorem 47 to the dual graph $G$ of the region $E_{x, y, z}^{(1)}(\mathbf{a} ; \mathbf{c} ; \mathbf{b})$ with the four vertices $u, v, w, s$ chosen as in Figure 6. In particular, the unit triangle corresponding to $u$ is the shaded one on the northeast corner of the region, the $v$-triangle is appended to the left of the right fern, the $w$-triangle is at the east corner of the region, and the $s$-triangle is at the southwest corner.

Let us consider the region corresponding to the graph $G-\{u, v, w, s\}$ (see Figure 15(b)). The removal of the $u^{-}, w_{-}, s$-triangles yields several forced lozenges on the region's boundary. The removal of the $v$-triangle yields forced lozenges on the side of the last down-pointing triangle of the $b$-fern. After removing these forced lozenges, we get an $R^{\leftarrow}$ type region considered in Theorem 2.3 of [31]. We note that Figure 15(c) illustrates the case when the $b$-fern has an even number of triangles, i.e., it ends with a down-pointing triangle. In the case $b$-fern has an odd number of triangles, we can regard that the $b$-fern 
ends with a down-pointing triangle of side-length 0 . In particular, we obtain the region $R_{x, y-1, z-1}^{\leftarrow}\left(\mathbf{a} ; \mathbf{c} ; \mathbf{b}^{+1}\right)$, where $\mathbf{b}^{+1}$ denotes the sequence obtained from $\mathbf{b}$ by adding 1 to the last term if $\mathbf{b}$ has an even number of terms, and in the case $\mathbf{b}$ has an odd number of terms, we include a new term 1 to the end of the sequence.

We note that, besides changing the side-lengths of the base hexagon and the $b$-fern, the removal of forced lozenges may change the center of the region. As a consequence, it may change the type of our region, in particular, from $E^{(1)}$ to $R^{\leftarrow}$ in this case. Since the removal of the forced lozenges does not change the tiling number, we get

$$
\mathrm{M}(G-\{u, v, w, s\})=\mathrm{M}\left(R_{x, y-1, z-1}^{\leftarrow}\left(\mathbf{a} ; \mathbf{c} ; \mathbf{b}^{+1}\right)\right) .
$$

By considering forced lozenges yields by the removal of $u-, v-, w-, s$-triangles as in Figures 15(d), (e) and (f), we have respectively

$$
\begin{aligned}
& \mathrm{M}(G-\{w, s\})=\mathrm{M}\left(G_{x, y-1, z}^{(1)}(\mathbf{a} ; \mathbf{c} ; \mathbf{b})\right), \\
& \mathrm{M}(G-\{u, s\})=\mathrm{M}\left(E_{x+1, y, z-1}^{(1)}(\mathbf{a} ; \mathbf{c} ; \mathbf{b})\right)
\end{aligned}
$$

and

$$
\mathrm{M}(G-\{v, w\})=\mathrm{M}\left(R_{x-1, y, z}^{\leftarrow}\left(\mathbf{a} ; \mathbf{c} ; \mathbf{b}^{+1}\right)\right)
$$

Finally, we remove forced lozenges from the region corresponding to $G-\{u, v\}, 180^{\circ}$ rotate the resulting region, and obtain

$$
\mathrm{M}(G-\{u, v\})=\mathrm{M}\left(K_{x, y-1, z-1}^{(1)}\left(\mathbf{b}^{+1} ; \overline{\mathbf{c}} ; \mathbf{a}\right)\right),
$$

where $\overline{\mathbf{c}}$ is the sequence obtained from $\mathbf{c}$ by reverting the order of its terms if $\mathbf{c}$ has an even number of terms, otherwise, we revert $\mathbf{c}$ and include a new 0 term in the beginning (see Figure 15(c)).

Plugging equations (42)-(46) into the equation in Kuo's Theorem 47, we have the following $E^{(1)}$-recurrence for $a \leqslant b$ :

$$
\begin{aligned}
\mathrm{M}\left(E_{x, y, z}^{(1)}(\mathbf{a} ; \mathbf{c} ; \mathbf{b})\right) \mathrm{M}\left(R_{x, y-1, z-1}^{\leftarrow}\left(\mathbf{a} ; \mathbf{c} ; \mathbf{b}^{+1}\right)\right)= \\
\mathrm{M}\left(K_{x, y-1, z-1}^{(1)}\left(\mathbf{b}^{+1} ; \overline{\mathbf{c}} ; \mathbf{a}\right)\right) \mathrm{M}\left(G_{x, y-1, z}^{(1)}(\mathbf{a} ; \mathbf{c} ; \mathbf{b})\right) \\
\quad+\mathrm{M}\left(E_{x+1, y, z-1}^{(1)}(\mathbf{a} ; \mathbf{c} ; \mathbf{b})\right) \mathrm{M}\left(R_{x-1, y-1, z}^{\leftarrow}\left(\mathbf{a} ; \mathbf{c} ; \mathbf{b}^{+1}\right)\right) .
\end{aligned}
$$

Working similarly for the case $a>b$, we get a slightly different $E^{(1)}$-recurrence:

$$
\begin{aligned}
\mathrm{M}\left(E_{x, y, z}^{(1)}(\mathbf{a} ; \mathbf{c} ; \mathbf{b})\right) \mathrm{M}\left(R_{x, y, z-1}^{\leftarrow}\left(\mathbf{a} ; \mathbf{c} ; \mathbf{b}^{+1}\right)\right)= \\
\operatorname{M}\left(K_{x, y, z-1}^{(1)}\left(\mathbf{b}^{+1} ; \overline{\mathbf{c}} ; \mathbf{a}\right)\right) \mathrm{M}\left(G_{x, y-1, z}^{(1)}(\mathbf{a} ; \mathbf{c} ; \mathbf{b})\right) \\
\quad+\mathrm{M}\left(E_{x+1, y, z-1}^{(1)}(\mathbf{a} ; \mathbf{c} ; \mathbf{b})\right) \mathrm{M}\left(R_{x-1, y, z}^{\leftarrow}\left(\mathbf{a} ; \mathbf{c} ; \mathbf{b}^{+1}\right)\right) .
\end{aligned}
$$

Applying Kuo condensation to the dual graph $G$ of the region $E_{x, y, z}^{(2)}(\mathbf{a} ; \mathbf{c} ; \mathbf{b})$ with the same choice of the four vertices $u, v, w, s$ as above, we get the $E^{(2)}$-recurrence for $a \leqslant b$ as

$$
\mathrm{M}\left(E_{x, y, z}^{(2)}(\mathbf{a} ; \mathbf{c} ; \mathbf{b})\right) \mathrm{M}\left(F_{x, y-1, z-1}^{(1)}\left(\mathbf{b}^{+1} ; \overline{\mathbf{c}} ; \mathbf{a}\right)\right)=
$$




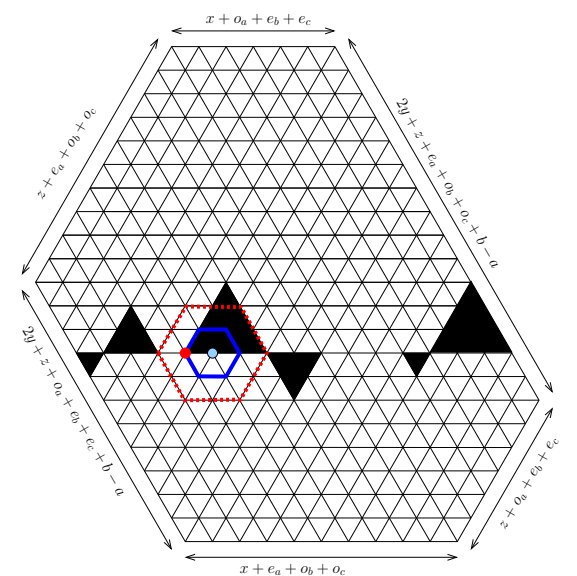

(a)

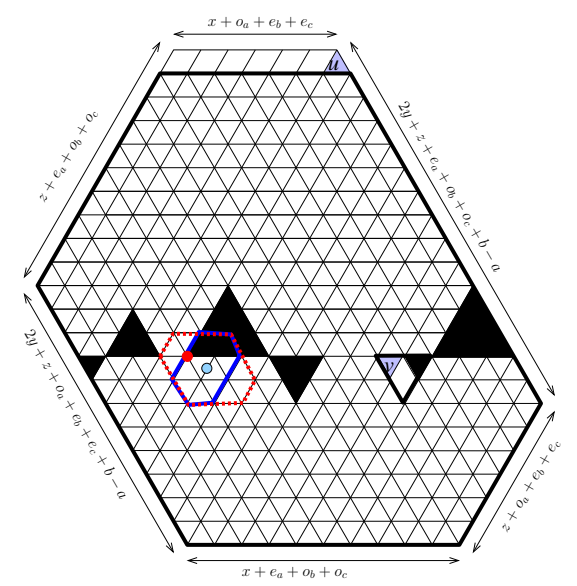

(c)

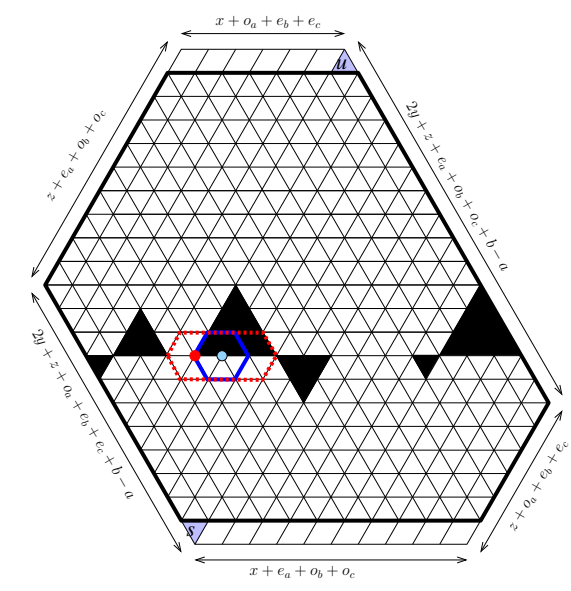

(e)

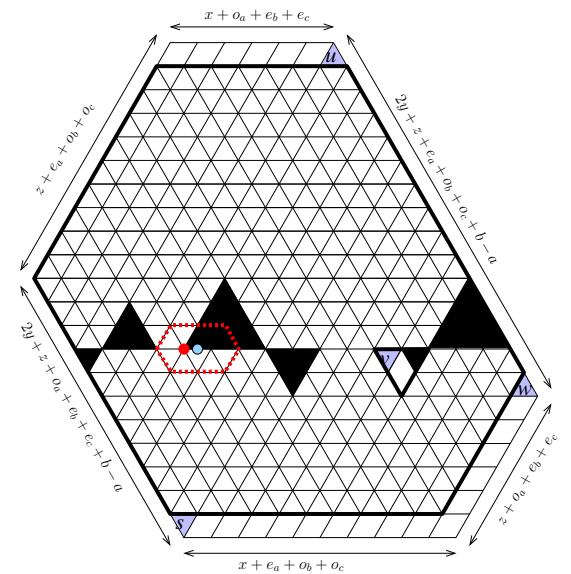

(b)

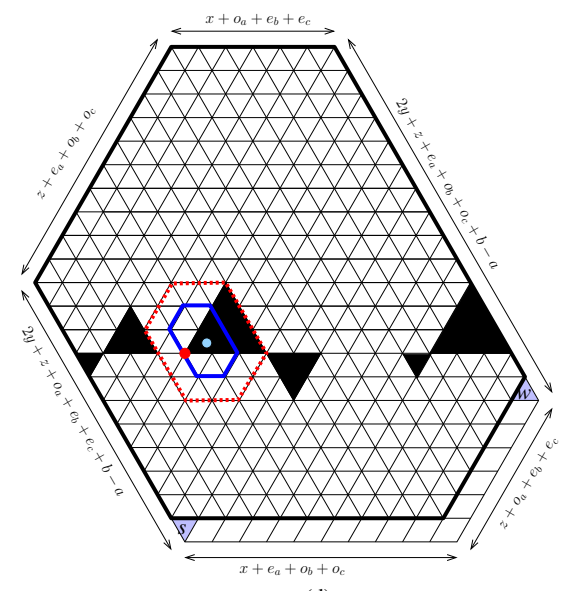

(d)

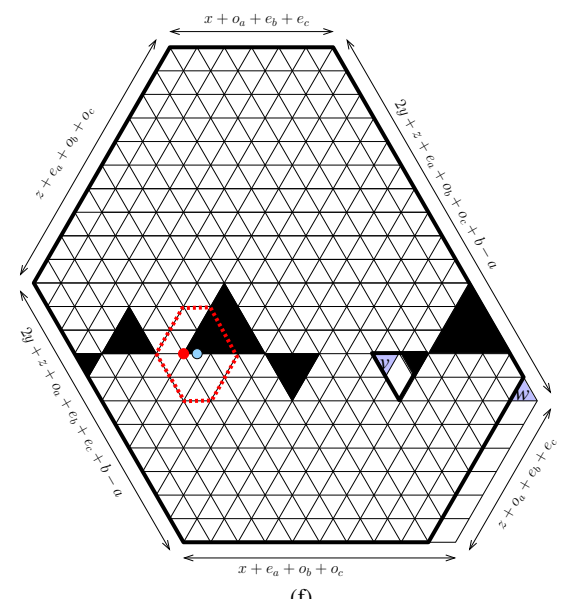

(f)

Figure 15: Obtaining a recurrence for $E^{(1)}$-type regions with $a \leqslant b$. Kuo condensation is applied to the region $E_{2,2,2}^{(1)}(1,2 ; 3,2 ; 3,1)$ (picture (a)) as shown on the picture (b).

$$
\mathrm{M}\left(K_{x, y-1, z-1}^{(2)}\left(\mathbf{b}^{+1} ; \overline{\mathbf{c}} ; \mathbf{a}\right)\right) \mathrm{M}\left(R_{x, y-1, z}^{\nwarrow}(\mathbf{a} ; \mathbf{c} ; \mathbf{b})\right)
$$




$$
+\mathrm{M}\left(E_{x+1, y, z-1}^{(2)}(\mathbf{a} ; \mathbf{c} ; \mathbf{b})\right) \mathrm{M}\left(F_{x-1, y-1, z}^{(1)}\left(\mathbf{b}^{+1} ; \overline{\mathbf{c}} ; \mathbf{a}\right)\right)
$$

and the $E^{(2)}$-recurrence for $a>b$ :

$$
\begin{aligned}
\mathrm{M}\left(E_{x, y, z}^{(2)}(\mathbf{a} ; \mathbf{c} ; \mathbf{b})\right) \mathrm{M}( & \left.F_{x, y, z-1}^{(1)}\left(\mathbf{b}^{+1} ; \overline{\mathbf{c}} ; \mathbf{a}\right)\right)= \\
\mathrm{M}( & \left.K_{x, y, z-1}^{(2)}\left(\mathbf{b}^{+1} ; \overline{\mathbf{c}} ; \mathbf{a}\right)\right) \mathrm{M}\left(R_{x, y-1, z}^{\nwarrow}(\mathbf{a} ; \mathbf{c} ; \mathbf{b})\right) \\
& +\mathrm{M}\left(E_{x+1, y, z-1}^{(2)}(\mathbf{a} ; \mathbf{c} ; \mathbf{b})\right) \mathrm{M}\left(F_{x-1, y, z}^{(1)}\left(\mathbf{b}^{+1} ; \overline{\mathbf{c}} ; \mathbf{a}\right)\right) .
\end{aligned}
$$

We note that the removal of forced lozenges from the region corresponding with $G-\{w, s\}$ yields an $R^{\nwarrow}$-type region in Theorem 2.4 in [31].

Finally, we have the recurrence for $E^{(6)}$-type regions by the same application of Kuo condensation. For $a \leqslant b$, we have

$$
\begin{aligned}
& \mathrm{M}\left(E_{x, y, z}^{(6)}(\mathbf{a} ; \mathbf{c} ; \mathbf{b})\right) \mathrm{M}\left(F_{x, y-1, z-1}^{(1)}\left(\mathbf{a} ; \mathbf{c} ; \mathbf{b}^{+1}\right)\right)= \\
& \operatorname{M}\left(R_{x, y-1, z-1}^{\swarrow}\left(\mathbf{a} ; \mathbf{c} ; \mathbf{b}^{+1}\right)\right) \mathrm{M}\left(G_{x, y-1, z}^{(4)}(\mathbf{b} ; \overline{\mathbf{c}} ; \mathbf{a})\right) \\
& \quad+\operatorname{M}\left(E_{x+1, y, z-1}^{(6)}(\mathbf{a} ; \mathbf{c} ; \mathbf{b})\right) \mathrm{M}\left(F_{x-1, y-1, z}^{(1)}\left(\mathbf{a} ; \mathbf{c} ; \mathbf{b}^{+1}\right)\right),
\end{aligned}
$$

and for $a>b$

$$
\begin{aligned}
& \mathrm{M}\left(E_{x, y, z}^{(6)}(\mathbf{a} ; \mathbf{c} ; \mathbf{b})\right) \mathrm{M}\left(F_{x, y, z-1}^{(1)}\left(\mathbf{a} ; \mathbf{c} ; \mathbf{b}^{+1}\right)\right)= \\
& \mathrm{M}\left(R_{x, y, z-1}^{\swarrow}\left(\mathbf{a} ; \mathbf{c} ; \mathbf{b}^{+1}\right)\right) \mathrm{M}\left(G_{x, y-1, z}^{(4)}(\mathbf{b} ; \overline{\mathbf{c}} ; \mathbf{a})\right) \\
& \quad+\mathrm{M}\left(E_{x+1, y, z-1}^{(6)}(\mathbf{a} ; \mathbf{c} ; \mathbf{b})\right) \mathrm{M}\left(F_{x-1, y, z}^{(1)}\left(\mathbf{a} ; \mathbf{c} ; \mathbf{b}^{+1}\right)\right) .
\end{aligned}
$$

The region corresponding with $G-\{u, v\}$ is congruent with, after removing forced lozenges, a region of type $R^{\swarrow}$ in Theorem 2.5 of [31].

\subsection{Recurrences for $\boldsymbol{F}^{(i)}$-type regions}

We apply Kuo Theorem 47 to the dual graph $G$ of region $F_{x, y, z}^{(1)}(\mathbf{a} ; \mathbf{c} ; \mathbf{b})$, for $a>b$, with the same choice of the four vertices $u, v, w, s$ as in the case for $E^{(i)}$-type regions in the previous subsection (see Figure 7). By considering forced lozenges yielded by the removal of the $u_{-}, v_{-}, w_{-}, s$-triangles as in Figures 16(b)-(f), we get respectively

$$
\begin{gathered}
\mathrm{M}(G-\{u, v, w, s\})=\mathrm{M}\left(E_{x, y, z-1}^{(2)}\left(\mathbf{b}^{+1} ; \overline{\mathbf{c}} ; \mathbf{a}\right)\right) \\
\mathrm{M}(G-\{u, v\})=\mathrm{M}\left(R_{x, y+1, z-1}^{\nwarrow}\left(\mathbf{b}^{+1} ; \overline{\mathbf{c}} ; \mathbf{a}\right)\right) \\
\mathrm{M}(G-\{w, s\})=\mathrm{M}\left(K_{x, y-1, z}^{(2)}(\mathbf{a} ; \mathbf{c} ; \mathbf{b})\right) \\
\mathrm{M}(G-\{u, s\})=\mathrm{M}\left(F_{x+1, y, z-1}^{(1)}(\mathbf{a} ; \mathbf{c} ; \mathbf{b})\right)
\end{gathered}
$$




$$
\mathrm{M}(G-\{v, w\})=\mathrm{M}\left(E_{x-1, y, z}^{(2)}\left(\mathbf{b}^{+1} ; \overline{\mathbf{c}} ; \mathbf{a}\right)\right) .
$$

We note that the regions on the right-hand sides of (53), (54) and (57) obtained by $180^{\circ}$ rotating the leftover regions (i.e. the remaining regions after removing forced lozenges; these regions are illustrated as the ones with bold contour).

Plugging the above 5 equations into the equation in Kuo's Theorem 47, we get the $F^{(1)}$-recurrence for $a>b$

$$
\begin{aligned}
& \mathrm{M}\left(F_{x, y, z}^{(1)}(\mathbf{a} ; \mathbf{c} ; \mathbf{b})\right) \mathrm{M}\left(E_{x, y, z-1}^{(2)}\left(\mathbf{b}^{+1} ; \overline{\mathbf{c}} ; \mathbf{a}\right)\right)= \\
& \quad \mathrm{M}\left(R_{x, y+1, z-1}^{\nwarrow}\left(\mathbf{b}^{+1} ; \overline{\mathbf{c}} ; \mathbf{a}\right)\right) \mathrm{M}\left(K_{x, y-1, z}^{(2)}(\mathbf{a} ; \mathbf{c} ; \mathbf{b})\right) \\
& \quad+\operatorname{M}\left(F_{x+1, y, z-1}^{(1)}(\mathbf{a} ; \mathbf{c} ; \mathbf{b})\right) \mathrm{M}\left(E_{x-1, y, z}^{(2)}\left(\mathbf{b}^{+1} ; \overline{\mathbf{c}} ; \mathbf{a}\right)\right) .
\end{aligned}
$$

Working similarly for the case $a \leqslant b$, we have a 'sibling' recurrence for $a \leqslant b$

$$
\begin{aligned}
& \mathrm{M}\left(F_{x, y, z}^{(1)}(\mathbf{a} ; \mathbf{c} ; \mathbf{b})\right) \mathrm{M}\left(E_{x, y-1, z-1}^{(2)}\left(\mathbf{b}^{+1} ; \overline{\mathbf{c}} ; \mathbf{a}\right)\right)= \\
& \quad \mathrm{M}\left(R_{x, y, z-1}^{\nwarrow}\left(\mathbf{b}^{+1} ; \overline{\mathbf{c}} ; \mathbf{a}\right)\right) \mathrm{M}\left(K_{x, y-1, z}^{(2)}(\mathbf{a} ; \mathbf{c} ; \mathbf{b})\right) \\
& \quad+\mathrm{M}\left(F_{x+1, y, z-1}^{(1)}(\mathbf{a} ; \mathbf{c} ; \mathbf{b})\right) \mathrm{M}\left(E_{x-1, y-1, z}^{(2)}\left(\mathbf{b}^{+1} ; \overline{\mathbf{c}} ; \mathbf{a}\right)\right) .
\end{aligned}
$$

By a similar application of Kuo condensation, we have the following recurrences for other $F^{(i)}$-type regions. The $F^{(2)}$-recurrence for $a \leqslant b$ is

$$
\begin{aligned}
& \mathrm{M}\left(F_{x, y, z}^{(2)}(\mathbf{a} ; \mathbf{c} ; \mathbf{b})\right) \mathrm{M}\left(E_{x, y-1, z-1}^{(6)}\left(\mathbf{a} ; \mathbf{c} ; \mathbf{b}^{+1}\right)\right)= \\
& \operatorname{M}\left(G_{x, y, z-1}^{(1)}\left(\mathbf{a} ; \mathbf{c} ; \mathbf{b}^{+1}\right)\right) \mathrm{M}\left(K_{x, y-1, z}^{(3)}(\mathbf{a} ; \mathbf{c} ; \mathbf{b})\right) \\
& \quad+\operatorname{M}\left(F_{x+1, y, z-1}^{(2)}(\mathbf{a} ; \mathbf{c} ; \mathbf{b})\right) \mathrm{M}\left(E_{x-1, y-1, z}^{(6)}\left(\mathbf{a} ; \mathbf{c} ; \mathbf{b}^{+1}\right)\right)
\end{aligned}
$$

and its $a>b$ counterpart is

$$
\begin{aligned}
& \mathrm{M}\left(F_{x, y, z}^{(2)}(\mathbf{a} ; \mathbf{c} ; \mathbf{b})\right) \mathrm{M}\left(E_{x, y, z-1}^{(6)}\left(\mathbf{a} ; \mathbf{c} ; \mathbf{b}^{+1}\right)\right)= \\
& \operatorname{M}\left(G_{x, y+1, z-1}^{(1)}\left(\mathbf{a} ; \mathbf{c} ; \mathbf{b}^{+1}\right)\right) \mathrm{M}\left(K_{x, y-1, z}^{(3)}(\mathbf{a} ; \mathbf{c} ; \mathbf{b})\right) \\
& \quad+\mathrm{M}\left(F_{x+1, y, z-1}^{(2)}(\mathbf{a} ; \mathbf{c} ; \mathbf{b})\right) \mathrm{M}\left(E_{x-1, y, z}^{(6)}\left(\mathbf{a} ; \mathbf{c} ; \mathbf{b}^{+1}\right)\right) .
\end{aligned}
$$

Next, we have the $F^{(3)}$-recurrences:

$$
\begin{aligned}
& \mathrm{M}\left(F_{x, y, z}^{(3)}(\mathbf{a} ; \mathbf{c} ; \mathbf{b})\right) \mathrm{M}\left(E_{x, y-1, z-1}^{(1)}\left(\mathbf{a} ; \mathbf{c} ; \mathbf{b}^{+1}\right)\right)= \\
& \mathrm{M}\left(G_{x, y-1, z-1}^{(2)}\left(\mathbf{a} ; \mathbf{c} ; \mathbf{b}^{+1}\right)\right) \mathrm{M}\left(K_{x, y-1, z}^{(4)}(\mathbf{a} ; \mathbf{c} ; \mathbf{b})\right) \\
& \quad+\mathrm{M}\left(F_{x+1, y, z-1}^{(3)}(\mathbf{a} ; \mathbf{c} ; \mathbf{b})\right) \mathrm{M}\left(E_{x-1, y-1, z}^{(1)}\left(\mathbf{a} ; \mathbf{c} ; \mathbf{b}^{+1}\right)\right)
\end{aligned}
$$

if $a \leqslant b$, and

$$
\begin{aligned}
& \mathrm{M}\left(F_{x, y, z}^{(3)}(\mathbf{a} ; \mathbf{c} ; \mathbf{b})\right) \mathrm{M}\left(E_{x, y, z-1}^{(1)}\left(\mathbf{a} ; \mathbf{c} ; \mathbf{b}^{+1}\right)\right)= \\
& \quad \operatorname{M}\left(G_{x, y, z-1}^{(2)}\left(\mathbf{a} ; \mathbf{c} ; \mathbf{b}^{+1}\right)\right) \mathrm{M}\left(K_{x, y-1, z}^{(4)}(\mathbf{a} ; \mathbf{c} ; \mathbf{b})\right)
\end{aligned}
$$



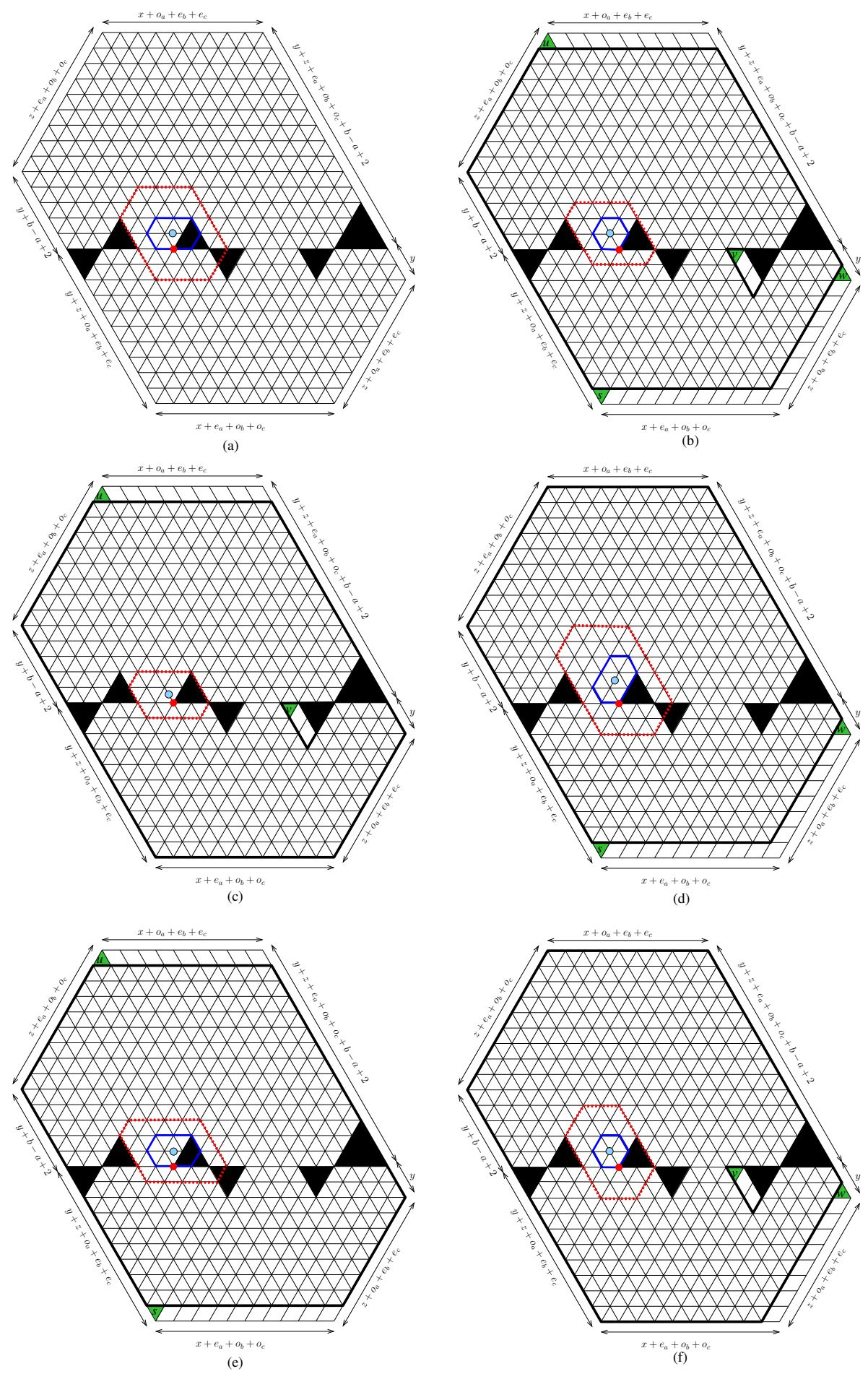

Figure 16: Obtaining a recurrence for $F^{(1)}$-type regions with $a \leqslant b$. Kuo condensation is applied to the region $F_{3,2,2}^{(1)}(2,2 ; 2,2 ; 3,2)$ (picture (a)) as shown on the picture (b). 


$$
+\mathrm{M}\left(F_{x+1, y, z-1}^{(3)}(\mathbf{a} ; \mathbf{c} ; \mathbf{b})\right) \mathrm{M}\left(E_{x-1, y, z}^{(1)}\left(\mathbf{a} ; \mathbf{c} ; \mathbf{b}^{+1}\right)\right)
$$

if $a>b$.

The last recurrence in this subsection is the $F^{(4)}$-recurrences below. For $a \leqslant b$, we have

$$
\begin{aligned}
& \mathrm{M}\left(F_{x, y, z}^{(4)}(\mathbf{a} ; \mathbf{c} ; \mathbf{b})\right) \mathrm{M}\left(E_{x, y-1, z-1}^{(2)}\left(\mathbf{a} ; \mathbf{c} ; \mathbf{b}^{+1}\right)\right)= \\
& \operatorname{M}\left(G_{x, y-1, z-1}^{(3)}\left(\mathbf{a} ; \mathbf{c} ; \mathbf{b}^{+1}\right)\right) \mathrm{M}\left(K_{x, y, z}^{(1)}(\mathbf{b} ; \overline{\mathbf{c}} ; \mathbf{a})\right) \\
& \quad+\mathrm{M}\left(F_{x+1, y, z-1}^{(4)}(\mathbf{a} ; \mathbf{c} ; \mathbf{b})\right) \mathrm{M}\left(E_{x-1, y-1, z}^{(2)}\left(\mathbf{a} ; \mathbf{c} ; \mathbf{b}^{+1}\right)\right)
\end{aligned}
$$

and for $a>b$

$$
\begin{aligned}
\mathrm{M}\left(F_{x, y, z}^{(4)}(\mathbf{a} ; \mathbf{c} ; \mathbf{b})\right) \mathrm{M}\left(E_{x, y, z-1}^{(2)}\left(\mathbf{a} ; \mathbf{c} ; \mathbf{b}^{+1}\right)\right)= \\
\mathrm{M}\left(G_{x, y, z-1}^{(3)}\left(\mathbf{a} ; \mathbf{c} ; \mathbf{b}^{+1}\right)\right) \mathrm{M}\left(K_{x, y, z}^{(1)}(\mathbf{b} ; \overline{\mathbf{c}} ; \mathbf{a})\right) \\
\quad+\operatorname{M}\left(F_{x+1, y, z-1}^{(4)}(\mathbf{a} ; \mathbf{c} ; \mathbf{b})\right) \operatorname{M}\left(E_{x-1, y, z}^{(2)}\left(\mathbf{a} ; \mathbf{c} ; \mathbf{b}^{+1}\right)\right) .
\end{aligned}
$$

\subsection{Recurrences for $G^{(i)}$-type regions}

For the $G^{(i)}$-type regions, we still apply Kuo's Theorem 47, however the four vertices $u, v, w, s$ are chosen differently, as in Figure 8. In particular, the $u$ - and $s$-triangles are the shaded ones appended to the ends of the right and left ferns, respectively. The $v$ and $w$-triangles are located at the east and southwest corners of the region, respectively.

Let us consider the dual graph $G$ of the region $G_{x, y, z}^{(2)}(\mathbf{a} ; \mathbf{c} ; \mathbf{b})$ in the case $a>b$. Working on forced lozenges as in Figures 17(b)-(f), we have respectively

$$
\begin{gathered}
\mathrm{M}(G-\{u, v, w, s\})=\mathrm{M}\left(E_{x-1, y, z-1}^{(1)}\left(\mathbf{a}^{+1} ; \mathbf{c} ; \mathbf{b}^{+1}\right)\right), \\
\mathrm{M}(G-\{u, v\})=\mathrm{M}\left(K_{x-1, y, z}^{(1)}\left(\mathbf{b}^{+1} ; \overline{\mathbf{c}} ; \mathbf{a}\right)\right), \\
\mathrm{M}(G-\{w, s\})=\mathrm{M}\left(F_{x, y, z-1}^{(3)}\left(\mathbf{a}^{+1} ; \mathbf{c} ; \mathbf{b}\right)\right), \\
\mathrm{M}(G-\{u, s\})=\mathrm{M}\left(G_{x-1, y, z-1}^{(2)}\left(\mathbf{a}^{+1} ; \mathbf{c} ; \mathbf{b}^{+1}\right)\right), \\
\mathrm{M}(G-\{v, w\})=\mathrm{M}\left(E_{x, y, z}^{(1)}(\mathbf{a} ; \mathbf{c} ; \mathbf{b})\right) .
\end{gathered}
$$

We note that the region on the right-hand side of (67) is obtained from the leftover region (the one restricted by the bold contour in Figure $17(\mathrm{c})$ ) by a $180^{\circ}$-rotation.

Plugging the above 5 equalities into the equation in Kuo's Theorem 47, we get the $G^{(2)}$-recurrences for $a>b$ :

$$
\mathrm{M}\left(G_{x, y, z}^{(2)}(\mathbf{a} ; \mathbf{c} ; \mathbf{b})\right) \mathrm{M}\left(E_{x-1, y, z-1}^{(1)}\left(\mathbf{a}^{+1} ; \mathbf{c} ; \mathbf{b}^{+1}\right)\right)=
$$




$$
\begin{aligned}
& \mathrm{M}\left(K_{x-1, y, z}^{(1)}\left(\mathbf{b}^{+1} ; \overline{\mathbf{c}} ; \mathbf{a}\right)\right) \mathrm{M}\left(F_{x, y, z-1}^{(3)}\left(\mathbf{a}^{+1} ; \mathbf{c} ; \mathbf{b}\right)\right) \\
& \quad+\mathrm{M}\left(G_{x-1, y, z-1}^{(2)}\left(\mathbf{a}^{+1} ; \mathbf{c} ; \mathbf{b}^{+1}\right)\right) \mathrm{M}\left(E_{x, y, z}^{(1)}(\mathbf{a} ; \mathbf{c} ; \mathbf{b})\right) .
\end{aligned}
$$

Similarly, we get the $G^{(2)}$-recurrence for $a<b$

$$
\begin{aligned}
\mathrm{M}\left(G_{x, y, z}^{(2)}(\mathbf{a} ; \mathbf{c} ; \mathbf{b})\right) \mathrm{M}\left(E_{x-1, y, z-1}^{(1)}\left(\mathbf{a}^{+1} ; \mathbf{c} ; \mathbf{b}^{+1}\right)\right)= \\
\mathrm{M}\left(K_{x-1, y-1, z}^{(1)}\left(\mathbf{b}^{+1} ; \overline{\mathbf{c}} ; \mathbf{a}\right)\right) \mathrm{M}\left(F_{x, y+1, z-1}^{(3)}\left(\mathbf{a}^{+1} ; \mathbf{c} ; \mathbf{b}\right)\right) \\
+\mathrm{M}\left(G_{x-1, y, z-1}^{(2)}\left(\mathbf{a}^{+1} ; \mathbf{c} ; \mathbf{b}^{+1}\right)\right) \mathrm{M}\left(E_{x, y, z}^{(1)}(\mathbf{a} ; \mathbf{c} ; \mathbf{b})\right)
\end{aligned}
$$

and for for $a=b$

$$
\begin{aligned}
\mathrm{M}\left(G_{x, y, z}^{(2)}(\mathbf{a} ; \mathbf{c} ; \mathbf{b})\right) \mathrm{M}\left(E_{x-1, y, z-1}^{(1)}\left(\mathbf{a}^{+1} ; \mathbf{c} ; \mathbf{b}^{+1}\right)\right)= & \\
\mathrm{M}( & \left.K_{x-1, y-1, z}^{(1)}\left(\mathbf{b}^{+1} ; \overline{\mathbf{c}} ; \mathbf{a}\right)\right) \mathrm{M}\left(F_{x, y, z-1}^{(3)}\left(\mathbf{a}^{+1} ; \mathbf{c} ; \mathbf{b}\right)\right) \\
+ & \mathrm{M}\left(G_{x-1, y, z-1}^{(2)}\left(\mathbf{a}^{+1} ; \mathbf{c} ; \mathbf{b}^{+1}\right)\right) \mathrm{M}\left(E_{x, y, z}^{(1)}(\mathbf{a} ; \mathbf{c} ; \mathbf{b})\right) .
\end{aligned}
$$

The same application of Kuo condensation gives us the recurrence of other $G^{(i)}$-type regions.

The $G^{(1)}$-recurrence for $a<b$

$$
\begin{aligned}
& \mathrm{M}\left(G_{x, y, z}^{(1)}(\mathbf{a} ; \mathbf{c} ; \mathbf{b})\right) \mathrm{M}\left(E_{x-1, y-1, z-1}^{(6)}\left(\mathbf{a}^{+1} ; \mathbf{c} ; \mathbf{b}^{+1}\right)\right)= \\
& \operatorname{M}\left(R_{x-1, y-1, z}^{\swarrow}\left(\mathbf{a} ; \mathbf{c} ; \mathbf{b}^{+1}\right)\right) \mathrm{M}\left(F_{x, y, z-1}^{(2)}\left(\mathbf{a}^{+1} ; \mathbf{c} ; \mathbf{b}\right)\right) \\
& \quad+\mathrm{M}\left(G_{x-1, y, z-1}^{(1)}\left(\mathbf{a}^{+1} ; \mathbf{c} ; \mathbf{b}^{+1}\right)\right) \mathrm{M}\left(E_{x, y-1, z}^{(6)}(\mathbf{a} ; \mathbf{c} ; \mathbf{b})\right),
\end{aligned}
$$

for $a>b$

$$
\begin{aligned}
& \mathrm{M}\left(G_{x, y, z}^{(1)}(\mathbf{a} ; \mathbf{c} ; \mathbf{b})\right) \mathrm{M}\left(E_{x-1, y-1, z-1}^{(6)}\left(\mathbf{a}^{+1} ; \mathbf{c} ; \mathbf{b}^{+1}\right)\right)= \\
& \quad \mathrm{M}\left(R_{x-1, y, z}^{\swarrow}\left(\mathbf{a} ; \mathbf{c} ; \mathbf{b}^{+1}\right)\right) \mathrm{M}\left(F_{x, y-1, z-1}^{(2)}\left(\mathbf{a}^{+1} ; \mathbf{c} ; \mathbf{b}\right)\right) \\
& \quad+\mathrm{M}\left(G_{x-1, y, z-1}^{(1)}\left(\mathbf{a}^{+1} ; \mathbf{c} ; \mathbf{b}^{+1}\right)\right) \mathrm{M}\left(E_{x, y-1, z}^{(6)}(\mathbf{a} ; \mathbf{c} ; \mathbf{b})\right),
\end{aligned}
$$

and for $a=b$

$$
\begin{aligned}
& \mathrm{M}\left(G_{x, y, z}^{(1)}(\mathbf{a} ; \mathbf{c} ; \mathbf{b})\right) \mathrm{M}\left(E_{x-1, y-1, z-1}^{(6)}\left(\mathbf{a}^{+1} ; \mathbf{c} ; \mathbf{b}^{+1}\right)\right)= \\
& \operatorname{M}\left(R_{x-1, y-1, z}^{\swarrow}\left(\mathbf{a} ; \mathbf{c} ; \mathbf{b}^{+1}\right)\right) \mathrm{M}\left(F_{x, y-1, z-1}^{(2)}\left(\mathbf{a}^{+1} ; \mathbf{c} ; \mathbf{b}\right)\right) \\
& \quad+\operatorname{M}\left(G_{x-1, y, z-1}^{(1)}\left(\mathbf{a}^{+1} ; \mathbf{c} ; \mathbf{b}^{+1}\right)\right) \mathrm{M}\left(E_{x, y-1, z}^{(6)}(\mathbf{a} ; \mathbf{c} ; \mathbf{b})\right) .
\end{aligned}
$$

The $G^{(3)}$-recurrence for $a<b$ is

$$
\begin{aligned}
\mathrm{M}\left(G_{x, y, z}^{(3)}(\mathbf{a} ; \mathbf{c} ; \mathbf{b})\right) \mathrm{M}\left(E_{x-1, y, z-1}^{(2)}\left(\mathbf{a}^{+1} ; \mathbf{c} ; \mathbf{b}^{+1}\right)\right)= & \\
\mathrm{M}( & \left.K_{x-1, y-1, z}^{(2)}\left(\mathbf{b}^{+1} ; \overline{\mathbf{c}} ; \mathbf{a}\right)\right) \mathrm{M}\left(F_{x, y+1, z-1}^{(4)}\left(\mathbf{a}^{+1} ; \mathbf{c} ; \mathbf{b}\right)\right) \\
+ & \mathrm{M}\left(G_{x-1, y, z-1}^{(3)}\left(\mathbf{a}^{+1} ; \mathbf{c} ; \mathbf{b}^{+1}\right)\right) \mathrm{M}\left(E_{x, y, z}^{(2)}(\mathbf{a} ; \mathbf{c} ; \mathbf{b})\right) .
\end{aligned}
$$



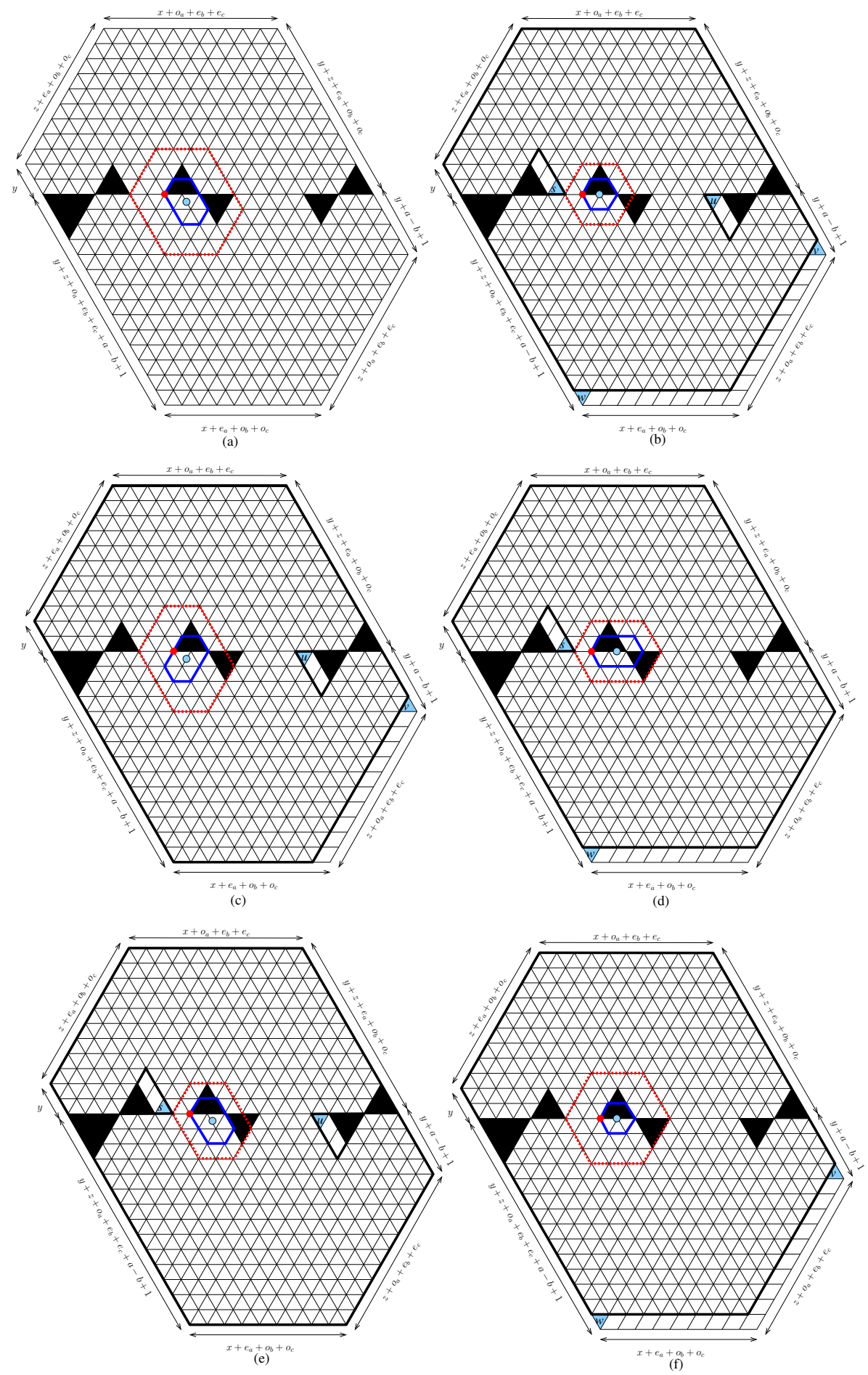

Figure 17: Obtaining a recurrence for $G^{(2)}$-type regions with $a>b$. Kuo condensation is applied to the region $G_{3,3,2}^{(2)}(3,2 ; 2,2 ; 2,2)$ (picture (a)) as shown on the picture (b). 
When $a>b$, this recurrence becomes

$$
\begin{aligned}
& \mathrm{M}\left(G_{x, y, z}^{(3)}(\mathbf{a} ; \mathbf{c} ; \mathbf{b})\right) \mathrm{M}\left(E_{x-1, y, z-1}^{(2)}\left(\mathbf{a}^{+1} ; \mathbf{c} ; \mathbf{b}^{+1}\right)\right)= \\
& \operatorname{M}\left(K_{x-1, y, z}^{(2)}\left(\mathbf{b}^{+1} ; \overline{\mathbf{c}} ; \mathbf{a}\right)\right) \mathrm{M}\left(F_{x, y, z-1}^{(4)}\left(\mathbf{a}^{+1} ; \mathbf{c} ; \mathbf{b}\right)\right) \\
& \quad+\mathrm{M}\left(G_{x-1, y, z-1}^{(3)}\left(\mathbf{a}^{+1} ; \mathbf{c} ; \mathbf{b}^{+1}\right)\right) \mathrm{M}\left(E_{x, y, z}^{(2)}(\mathbf{a} ; \mathbf{c} ; \mathbf{b})\right),
\end{aligned}
$$

and when $a=b$ we have

$$
\begin{aligned}
\mathrm{M}\left(G_{x, y, z}^{(3)}(\mathbf{a} ; \mathbf{c} ; \mathbf{b})\right) \mathrm{M}\left(E_{x-1, y, z-1}^{(2)}\left(\mathbf{a}^{+1} ; \mathbf{c} ; \mathbf{b}^{+1}\right)\right)= & \\
\mathrm{M}( & \left.K_{x-1, y-1, z}^{(2)}\left(\mathbf{b}^{+1} ; \overline{\mathbf{c}} ; \mathbf{a}\right)\right) \mathrm{M}\left(F_{x, y, z-1}^{(4)}\left(\mathbf{a}^{+1} ; \mathbf{c} ; \mathbf{b}\right)\right) \\
+ & \mathrm{M}\left(G_{x-1, y, z-1}^{(3)}\left(\mathbf{a}^{+1} ; \mathbf{c} ; \mathbf{b}^{+1}\right)\right) \mathrm{M}\left(E_{x, y, z}^{(2)}(\mathbf{a} ; \mathbf{c} ; \mathbf{b})\right) .
\end{aligned}
$$

Finally, the application of Kuo condensation gives the $G^{(4)}$-recurrence as

$$
\begin{aligned}
\mathrm{M}\left(G_{x, y, z}^{(4)}(\mathbf{a} ; \mathbf{c} ; \mathbf{b})\right) \mathrm{M}\left(E_{x-1, y, z-1}^{(6)}\left(\mathbf{b}^{+1} ; \overline{\mathbf{c}} ; \mathbf{a}^{+1}\right)\right)= \\
\mathrm{M}\left(K_{x-1, y-1, z}^{(3)}\left(\mathbf{b}^{+1} ; \overline{\mathbf{c}} ; \mathbf{a}\right)\right) \mathrm{M}\left(F_{x, y+1, z-1}^{(1)}\left(\mathbf{b} ; \mathbf{c} ; \mathbf{a}^{+1}\right)\right) \\
\quad+\mathrm{M}\left(G_{x-1, y, z-1}^{(4)}\left(\mathbf{a}^{+1} ; \mathbf{c} ; \mathbf{b}^{+1}\right)\right) \mathrm{M}\left(E_{x, y, z}^{(6)}(\mathbf{b} ; \overline{\mathbf{c}} ; \mathbf{a})\right)
\end{aligned}
$$

for $a<b$,

$$
\begin{aligned}
\mathrm{M}\left(G_{x, y, z}^{(4)}(\mathbf{a} ; \mathbf{c} ; \mathbf{b})\right) \mathrm{M}\left(E_{x-1, y, z-1}^{(6)}\left(\mathbf{b}^{+1} ; \overline{\mathbf{c}} ; \mathbf{a}^{+1}\right)\right)= \\
\mathrm{M}\left(K_{x-1, y, z}^{(3)}\left(\mathbf{b}^{+1} ; \overline{\mathbf{c}} ; \mathbf{a}\right)\right) \mathrm{M}\left(F_{x, y, z-1}^{(1)}\left(\mathbf{b} ; \mathbf{c} ; \mathbf{a}^{+1}\right)\right) \\
\quad+\mathrm{M}\left(G_{x-1, y, z-1}^{(4)}\left(\mathbf{a}^{+1} ; \mathbf{c} ; \mathbf{b}^{+1}\right)\right) \mathrm{M}\left(E_{x, y, z}^{(6)}(\mathbf{b} ; \overline{\mathbf{c}} ; \mathbf{a})\right)
\end{aligned}
$$

for $a>b$, and

$$
\begin{aligned}
\mathrm{M}\left(G_{x, y, z}^{(4)}(\mathbf{a} ; \mathbf{c} ; \mathbf{b})\right) \mathrm{M}\left(E_{x-1, y, z-1}^{(6)}\left(\mathbf{b}^{+1} ; \overline{\mathbf{c}} ; \mathbf{a}^{+1}\right)\right)= \\
\mathrm{M}\left(K_{x-1, y-1, z}^{(3)}\left(\mathbf{b}^{+1} ; \overline{\mathbf{c}} ; \mathbf{a}\right)\right) \mathrm{M}\left(F_{x, y, z-1}^{(1)}\left(\mathbf{b} ; \mathbf{c} ; \mathbf{a}^{+1}\right)\right) \\
\quad+\mathrm{M}\left(G_{x-1, y, z-1}^{(4)}\left(\mathbf{a}^{+1} ; \mathbf{c} ; \mathbf{b}^{+1}\right)\right) \mathrm{M}\left(E_{x, y, z}^{(6)}(\mathbf{b} ; \overline{\mathbf{c}} ; \mathbf{a})\right)
\end{aligned}
$$

for $a=b$.

\subsection{Recurrences for $K^{(i)}$-type regions}

For the case of $K^{(i)}$-regions, we still apply Kuo's Theorem 47 with the three vertices $u, v, w$ chosen similarly to that in the cases of $E^{(i)}$ - and $F^{(i)}$-type regions; the only difference is that the $s$-triangle is now located at the west corner (see Figure 9 ). We now consider the $K^{(1)}$-type regions with $a>b$.

Let $G$ be the dual graph of the region $K_{x, y, z}^{(1)}(\mathbf{a} ; \mathbf{c} ; \mathbf{b})$ (for $a>b$ ). Considering forced lozenges, we get

$$
\mathrm{M}(G-\{u, v, w, s\})=\mathrm{M}\left(E_{x-1, y, z}^{(1)}\left(\mathbf{b}^{+1} ; \overline{\mathbf{c}} ; \mathbf{a}\right)\right),
$$




$$
\begin{gathered}
\mathrm{M}(G-\{u, v\})=\mathrm{M}\left(E_{x, y+1, z-1}^{(1)}\left(\mathbf{b}^{+1} ; \overline{\mathbf{c}} ; \mathbf{a}\right)\right) \\
\mathrm{M}(G-\{w, s\})=\mathrm{M}\left(K_{x-1, y-1, z+1}^{(1)}(\mathbf{a} ; \mathbf{c} ; \mathbf{b})\right) \\
\mathrm{M}(G-\{u, s\})=\mathrm{M}\left(R_{x, y, z}^{\leftarrow}(\mathbf{a} ; \mathbf{c} ; \mathbf{b})\right) \\
\mathrm{M}(G-\{v, w\})=\mathrm{M}\left(G_{x-1, y, z}^{(2)}\left(\mathbf{b}^{+1} ; \overline{\mathbf{c}} ; \mathbf{a}\right)\right) .
\end{gathered}
$$

We need to $180^{\circ}$-rotate the leftover regions in the case of $(83),(84),(87)$. By equations (83)-(87) and Theorem 47, we have the $K^{(1)}$-recurrence for $a>b$ :

$$
\begin{aligned}
\mathrm{M}\left(K_{x, y, z}^{(1)}(\mathbf{a} ; \mathbf{c} ; \mathbf{b})\right) \mathrm{M}\left(E_{x-1, y, z}^{(1)}\left(\mathbf{b}^{+1} ; \overline{\mathbf{c}} ; \mathbf{a}\right)\right)= \\
\mathrm{M}\left(E_{x, y+1, z-1}^{(1)}\left(\mathbf{b}^{+1} ; \overline{\mathbf{c}} ; \mathbf{a}\right)\right) \mathrm{M}\left(K_{x-1, y-1, z+1}^{(1)}(\mathbf{a} ; \mathbf{c} ; \mathbf{b})\right) \\
\quad+\mathrm{M}\left(R_{x, y, z}^{\leftarrow}(\mathbf{a} ; \mathbf{c} ; \mathbf{b})\right) \mathrm{M}\left(G_{x-1, y, z}^{(2)}\left(\mathbf{b}^{+1} ; \overline{\mathbf{c}} ; \mathbf{a}\right)\right) .
\end{aligned}
$$

By a similar application of Kuo condensation, we get other recurrences:

The $K^{(1)}$-recurrence for $a \leqslant b$ :

$$
\begin{aligned}
\mathrm{M}\left(K_{x, y, z}^{(1)}(\mathbf{a} ; \mathbf{c} ; \mathbf{b})\right) \mathrm{M}\left(E_{x-1, y-1, z}^{(1)}\left(\mathbf{b}^{+1} ; \overline{\mathbf{c}} ; \mathbf{a}\right)\right)= \\
\mathrm{M}\left(E_{x, y, z-1}^{(1)}\left(\mathbf{b}^{+1} ; \overline{\mathbf{c}} ; \mathbf{a}\right)\right) \mathrm{M}\left(K_{x-1, y-1, z+1}^{(1)}(\mathbf{a} ; \mathbf{c} ; \mathbf{b})\right) \\
\quad+\mathrm{M}\left(R_{x, y, z}^{\leftarrow}(\mathbf{a} ; \mathbf{c} ; \mathbf{b})\right) \mathrm{M}\left(G_{x-1, y-1, z}^{(2)}\left(\mathbf{b}^{+1} ; \overline{\mathbf{c}} ; \mathbf{a}\right)\right) .
\end{aligned}
$$

The $K^{(2)}$-recurrence for $a \leqslant b$ :

$$
\begin{aligned}
\mathrm{M}\left(K_{x, y, z}^{(2)}(\mathbf{a} ; \mathbf{c} ; \mathbf{b})\right) \mathrm{M}\left(E_{x-1, y-1, z}^{(2)}\left(\mathbf{b}^{+1} ; \overline{\mathbf{c}} ; \mathbf{a}\right)\right)= \\
\mathrm{M}\left(E_{x, y, z-1}^{(2)}\left(\mathbf{b}^{+1} ; \overline{\mathbf{c}} ; \mathbf{a}\right)\right) \mathrm{M}\left(K_{x-1, y-1, z+1}^{(2)}(\mathbf{a} ; \mathbf{c} ; \mathbf{b})\right) \\
\quad+\mathrm{M}\left(F_{x, y, z}^{(1)}(\mathbf{a} ; \mathbf{c} ; \mathbf{b})\right) \mathrm{M}\left(G_{x-1, y-1, z}^{(3)}\left(\mathbf{b}^{+1} ; \overline{\mathbf{c}} ; \mathbf{a}\right)\right) .
\end{aligned}
$$

The $K^{(2)}$-recurrence for $a>b$ (illustrated in Figure 18):

$$
\begin{aligned}
\mathrm{M}\left(K_{x, y, z}^{(2)}(\mathbf{a} ; \mathbf{c} ; \mathbf{b})\right) \mathrm{M}\left(E_{x-1, y, z}^{(2)}\left(\mathbf{b}^{+1} ; \overline{\mathbf{c}} ; \mathbf{a}\right)\right)= \\
\mathrm{M}\left(E_{x, y+1, z-1}^{(2)}\left(\mathbf{b}^{+1} ; \overline{\mathbf{c}} ; \mathbf{a}\right)\right) \mathrm{M}\left(K_{x-1, y-1, z+1}^{(2)}(\mathbf{a} ; \mathbf{c} ; \mathbf{b})\right) \\
+\operatorname{M}\left(F_{x, y, z}^{(1)}(\mathbf{a} ; \mathbf{c} ; \mathbf{b})\right) \mathrm{M}\left(G_{x-1, y, z}^{(3)}\left(\mathbf{b}^{+1} ; \overline{\mathbf{c}} ; \mathbf{a}\right)\right) .
\end{aligned}
$$

The $K^{(3)}$-recurrence for $a \leqslant b$ :

$$
\begin{aligned}
\mathrm{M}\left(K_{x, y, z}^{(3)}(\mathbf{a} ; \mathbf{c} ; \mathbf{b})\right) \mathrm{M}\left(E_{x-1, y-1, z}^{(6)}\left(\mathbf{a} ; \mathbf{c} ; \mathbf{b}^{+1}\right)\right)= \\
\mathrm{M}\left(E_{x, y, z-1}^{(6)}\left(\mathbf{a} ; \mathbf{c} ; \mathbf{b}^{+1}\right)\right) \mathrm{M}\left(K_{x-1, y-1, z+1}^{(3)}(\mathbf{a} ; \mathbf{c} ; \mathbf{b})\right) \\
\quad+\operatorname{M}\left(F_{x, y, z}^{(2)}(\mathbf{a} ; \mathbf{c} ; \mathbf{b})\right) \mathrm{M}\left(G_{x-1, y-1, z}^{(4)}\left(\mathbf{b}^{+1} ; \overline{\mathbf{c}} ; \mathbf{a}\right)\right) .
\end{aligned}
$$



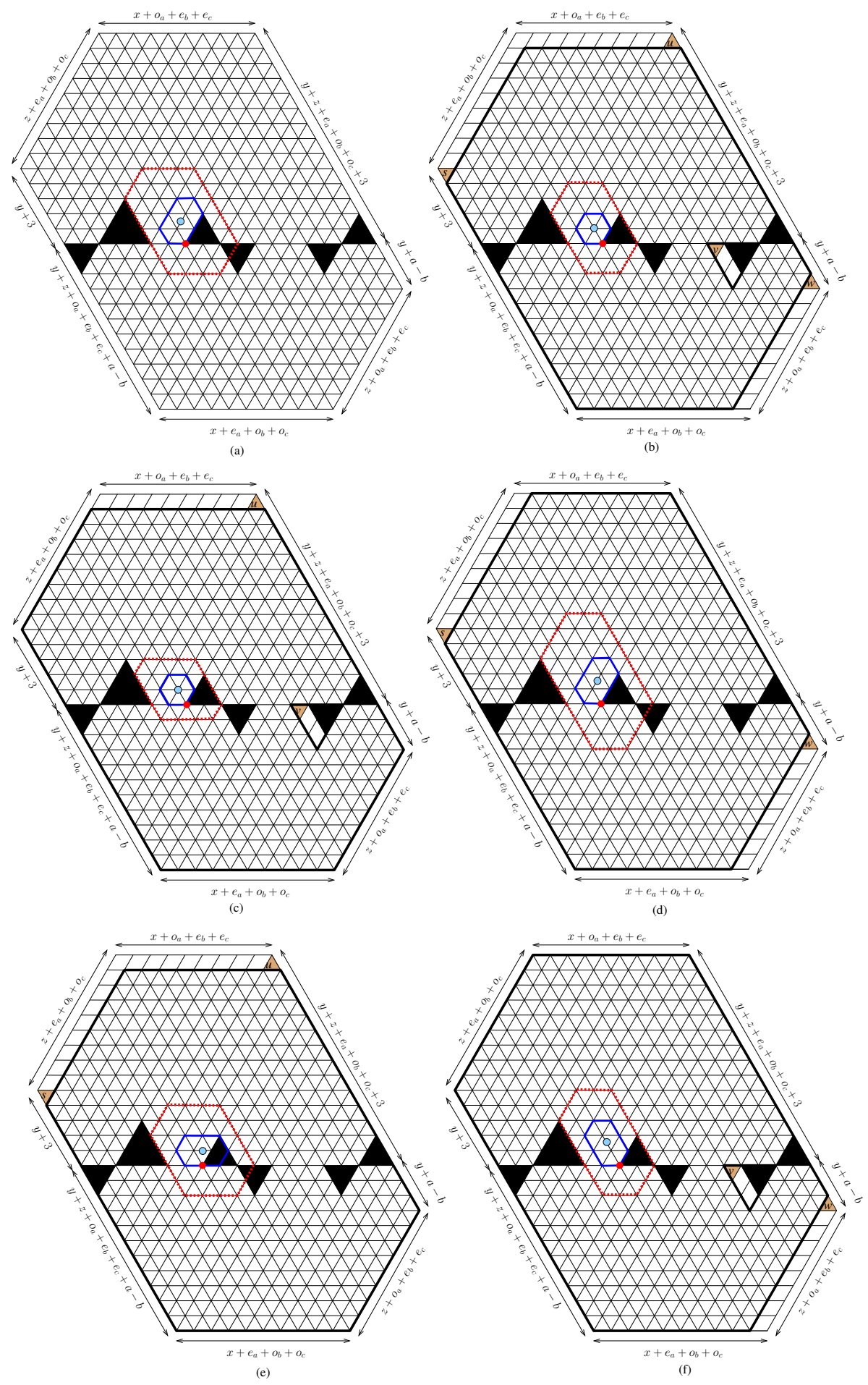

Figure 18: Obtaining a recurrence for $K^{(2)}$-type regions with $a>b$. Kuo condensation is applied to the region $K_{3,2,2}^{(2)}(2,3 ; 2,2 ; 2,2)$ (picture (a)) as shown on the picture (b). 
The $K^{(3)}$-recurrence for $a>b$ :

$$
\begin{aligned}
& \mathrm{M}\left(K_{x, y, z}^{(3)}(\mathbf{a} ; \mathbf{c} ; \mathbf{b})\right) \mathrm{M}\left(E_{x-1, y, z}^{(6)}\left(\mathbf{a} ; \mathbf{c} ; \mathbf{b}^{+1}\right)\right)= \\
& \mathrm{M}\left(E_{x, y+1, z-1}^{(6)}\left(\mathbf{a} ; \mathbf{c} ; \mathbf{b}^{+1}\right)\right) \mathrm{M}\left(K_{x-1, y-1, z+1}^{(3)}(\mathbf{a} ; \mathbf{c} ; \mathbf{b})\right) \\
& \quad+\mathrm{M}\left(F_{x, y, z}^{(2)}(\mathbf{a} ; \mathbf{c} ; \mathbf{b})\right) \mathrm{M}\left(G_{x-1, y, z}^{(4)}\left(\mathbf{b}^{+1} ; \overline{\mathbf{c}} ; \mathbf{a}\right)\right) .
\end{aligned}
$$

The $K^{(4)}$-recurrence for $a \leqslant b$ :

$$
\begin{aligned}
\mathrm{M}\left(K_{x, y, z}^{(4)}(\mathbf{a} ; \mathbf{c} ; \mathbf{b})\right) \mathrm{M}\left(E_{x-1, y-1, z}^{(1)}\left(\mathbf{a} ; \mathbf{c} ; \mathbf{b}^{+1}\right)\right)= \\
\operatorname{M}\left(E_{x, y, z-1}^{(1)}\left(\mathbf{a} ; \mathbf{c} ; \mathbf{b}^{+1}\right)\right) \mathrm{M}\left(K_{x-1, y-1, z+1}^{(4)}(\mathbf{a} ; \mathbf{c} ; \mathbf{b})\right) \\
+\operatorname{M}\left(F_{x, y, z}^{(3)}(\mathbf{a} ; \mathbf{c} ; \mathbf{b})\right) \operatorname{M}\left(G_{x-1, y-1, z}^{(1)}\left(\mathbf{a} ; \mathbf{c} ; \mathbf{b}^{+1}\right)\right) .
\end{aligned}
$$

The $K^{(4)}$-recurrence for $a>b$ :

$$
\begin{aligned}
\mathrm{M}\left(K_{x, y, z}^{(4)}(\mathbf{a} ; \mathbf{c} ; \mathbf{b})\right) \mathrm{M}\left(E_{x-1, y, z}^{(1)}\left(\mathbf{a} ; \mathbf{c} ; \mathbf{b}^{+1}\right)\right)= \\
\mathrm{M}\left(E_{x, y+1, z-1}^{(1)}\left(\mathbf{a} ; \mathbf{c} ; \mathbf{b}^{+1}\right)\right) \mathrm{M}\left(K_{x-1, y-1, z+1}^{(4)}(\mathbf{a} ; \mathbf{c} ; \mathbf{b})\right) \\
\quad+\mathrm{M}\left(F_{x, y, z}^{(3)}(\mathbf{a} ; \mathbf{c} ; \mathbf{b})\right) \mathrm{M}\left(G_{x-1, y, z}^{(1)}\left(\mathbf{a} ; \mathbf{c} ; \mathbf{b}^{+1}\right)\right) .
\end{aligned}
$$

\subsection{Recurrences for $\overline{\boldsymbol{E}}^{(i)}$-type regions}

We apply Theorem 47 to the $\bar{E}^{(1)}$ - and $\bar{E}^{(2)}$-type regions with the particular choice of the four vertices $u, v, w, s$, as shown in Figures 11(a) and (b), respectively. For the $\bar{E}^{(3)}$-type regions, we apply Theorem 48 as in Figure 11(c).

We now investigate $\bar{E}^{(1)}$-type regions with $a \leqslant b$. We also apply Kuo's Theorem 47 to the dual graph $G$ of the region $\bar{E}_{x, y, z}^{(1)}(\mathbf{a} ; \mathbf{c} ; \mathbf{b})$. Considering forced lozenges, we have

$$
\begin{gathered}
\mathrm{M}(G-\{u, v, w, s\})=\mathrm{M}\left(Q_{x, y, z-1}^{\leftarrow}\left(\mathbf{a} ; \mathbf{c} ; \mathbf{b}^{+1}\right)\right), \\
\mathrm{M}(G-\{u, v\})=\mathrm{M}\left(\bar{K}_{x, y, z-1}^{(5)}\left(\mathbf{a} ; \mathbf{c} ; \mathbf{b}^{+1}\right)\right), \\
\mathrm{M}(G-\{w, s\})=\mathrm{M}\left(\bar{G}_{x, y-1, z}^{(5)}\left(\mathbf{b} ; \mathbf{c}^{\leftrightarrow} ; \mathbf{a}\right)\right), \\
\mathrm{M}(G-\{u, s\})=\mathrm{M}\left(\bar{E}_{x+1, y, z-1}^{(1)}(\mathbf{a} ; \mathbf{c} ; \mathbf{b})\right), \\
\mathrm{M}(G-\{v, w\})=\mathrm{M}\left(Q_{x-1, y, z}^{\leftarrow}\left(\mathbf{a} ; \mathbf{c} ; \mathbf{b}^{+1}\right)\right) .
\end{gathered}
$$

The region of type $Q^{\leftarrow}$ was enumerated in Theorem 2.7 of [31]. Here, the sequence $\mathbf{c}^{\leftrightarrow}$ obtained by reverting the sequence $\mathbf{c}$ when it has an odd number of terms, otherwise, we revert and add a new 0 term in the beginning. More precisely, the leftover region after 
the removal of forced lozenges in (98) is not of our interests. We need to reflect the this region to get $\bar{G}_{x, y-1, z}^{(5)}\left(\mathbf{b} ; \mathbf{c}^{\leftrightarrow} ; \mathbf{a}\right)$.

Plugging the above five equations into that of Theorem 47, we obtain the $\bar{E}^{(1)}$ recurrence for $a \leqslant b$ :

$$
\begin{aligned}
& \mathrm{M}\left(\bar{E}_{x, y, z}^{(1)}(\mathbf{a} ; \mathbf{c} ; \mathbf{b})\right) \mathrm{M}\left(Q_{x, y, z-1}^{\leftarrow}\left(\mathbf{a} ; \mathbf{c} ; \mathbf{b}^{+1}\right)\right)= \\
& \quad \mathrm{M}\left(\bar{K}_{x, y, z-1}^{(5)}\left(\mathbf{a} ; \mathbf{c} ; \mathbf{b}^{+1}\right)\right) \mathrm{M}\left(\bar{G}_{x, y-1, z}^{(5)}\left(\mathbf{b} ; \mathbf{c}{ }^{\leftrightarrow} ; \mathbf{a}\right)\right) \\
& \quad+\mathrm{M}\left(\bar{E}_{x+1, y, z-1}^{(1)}(\mathbf{a} ; \mathbf{c} ; \mathbf{b})\right) \mathrm{M}\left(Q_{x-1, y, z}^{\leftarrow}\left(\mathbf{a} ; \mathbf{c} ; \mathbf{b}^{+1}\right)\right)
\end{aligned}
$$

Working out similarly, we get other recurrences:

The $\bar{E}^{(1)}$-recurrence for $a>b$

$$
\begin{aligned}
& \mathrm{M}\left(\bar{E}_{x, y, z}^{(1)}(\mathbf{a} ; \mathbf{c} ; \mathbf{b})\right) \mathrm{M}\left(Q_{x, y-1, z-1}^{\leftarrow}\left(\mathbf{a} ; \mathbf{c} ; \mathbf{b}^{+1}\right)\right)= \\
& \quad \mathrm{M}\left(\bar{K}_{x, y-1, z-1}^{(5)}\left(\mathbf{a} ; \mathbf{c} ; \mathbf{b}^{+1}\right)\right) \mathrm{M}\left(\bar{G}_{x, y-1, z}^{(5)}(\mathbf{b} ; \mathbf{c} \leftrightarrow ; \mathbf{a})\right) \\
& \quad+\operatorname{M}\left(\bar{E}_{x+1, y, z-1}^{(1)}(\mathbf{a} ; \mathbf{c} ; \mathbf{b})\right) \mathrm{M}\left(Q_{x-1, y-1, z}^{\leftarrow}\left(\mathbf{a} ; \mathbf{c} ; \mathbf{b}^{+1}\right)\right)
\end{aligned}
$$

The $\bar{E}^{(2)}$-recurrence for $a \leqslant b$

$$
\begin{aligned}
\mathrm{M}\left(\bar{E}_{x, y, z}^{(2)}(\mathbf{a} ; \mathbf{c} ; \mathbf{b})\right) \mathrm{M}\left(Q_{x-1, y, z-1}^{\nwarrow}\left(\mathbf{a}^{+1} ; \mathbf{c} ; \mathbf{b}^{+1}\right)\right)= \\
\mathrm{M}\left(\bar{F}_{x-1, y-1, z}^{(5)}\left(\mathbf{a} ; \mathbf{c} ; \mathbf{b}^{+1}\right)\right) \mathrm{M}\left(\bar{K}_{x, y+1, z-1}^{(5)}\left(\mathbf{a}^{+1} ; \mathbf{c} ; \mathbf{b}\right)\right) \\
\quad+\mathrm{M}\left(\bar{E}_{x-1, y, z-1}^{(2)}\left(\mathbf{a}^{+1} ; \mathbf{c} ; \mathbf{b}^{+1}\right)\right) \mathrm{M}\left(Q_{x, y, z}^{\nwarrow}(\mathbf{a} ; \mathbf{c} ; \mathbf{b})\right) .
\end{aligned}
$$

The $\bar{E}^{(2)}$-recurrence for $a>b$

$$
\begin{aligned}
\mathrm{M}\left(\bar{E}_{x, y, z}^{(2)}(\mathbf{a} ; \mathbf{c} ; \mathbf{b})\right) \mathrm{M}\left(Q_{x-1, y, z-1}^{\nwarrow}\left(\mathbf{a}^{+1} ; \mathbf{c} ; \mathbf{b}^{+1}\right)\right)= \\
\quad \mathrm{M}\left(\bar{F}_{x-1, y, z}^{(5)}\left(\mathbf{a} ; \mathbf{c} ; \mathbf{b}^{+1}\right)\right) \mathrm{M}\left(\bar{K}_{x, y, z-1}^{(5)}\left(\mathbf{a}^{+1} ; \mathbf{c} ; \mathbf{b}\right)\right) \\
\quad+\mathrm{M}\left(\bar{E}_{x-1, y, z-1}^{(2)}\left(\mathbf{a}^{+1} ; \mathbf{c} ; \mathbf{b}^{+1}\right)\right) \mathrm{M}\left(Q_{x, y, z}^{\nwarrow}(\mathbf{a} ; \mathbf{c} ; \mathbf{b})\right) .
\end{aligned}
$$

The $\bar{E}^{(3)}$-recurrence for $a \leqslant b$ (illustrated in Figure 19)

$$
\begin{aligned}
& \mathrm{M}\left(Q_{x, y+1, z-1}^{\nearrow}\left(\mathbf{a}^{+1} ; \mathbf{c} ; \mathbf{b}\right)\right) \mathrm{M}\left(\bar{G}_{x, y, z}^{(5)}(\mathbf{a} ; \mathbf{c} ; \mathbf{b})\right)= \\
& \quad \mathrm{M}\left(\bar{E}_{x, y, z}^{(3)}(\mathbf{a} ; \mathbf{c} ; \mathbf{b})\right) \mathrm{M}\left(Q_{x, y+1, z-1}^{\leftarrow}\left(\mathbf{b} ; \mathbf{c}^{\leftrightarrow} ; \mathbf{a}^{+1}\right)\right) \\
& \quad+\operatorname{M}\left(\bar{E}_{x+1, y, z-1}^{(3)}(\mathbf{a} ; \mathbf{c} ; \mathbf{b})\right) \mathrm{M}\left(Q_{x-1, y+1, z}^{\leftarrow}\left(\mathbf{b} ; \mathbf{c}^{\leftrightarrow} ; \mathbf{a}^{+1}\right)\right) .
\end{aligned}
$$

The $\bar{E}^{(3)}$-recurrence for $a>b$

$$
\begin{aligned}
& \mathrm{M}\left(Q_{x, y, z-1}^{\nearrow}\left(\mathbf{a}^{+1} ; \mathbf{c} ; \mathbf{b}\right)\right) \mathrm{M}\left(\bar{G}_{x, y, z}^{(5)}(\mathbf{a} ; \mathbf{c} ; \mathbf{b})\right)= \\
& \quad \mathrm{M}\left(\bar{E}_{x, y, z}^{(3)}(\mathbf{a} ; \mathbf{c} ; \mathbf{b})\right) \mathrm{M}\left(Q_{x, y, z-1}^{\leftarrow}\left(\mathbf{b} ; \mathbf{c}^{\leftrightarrow} ; \mathbf{a}^{+1}\right)\right) \\
& \quad+\mathrm{M}\left(\bar{E}_{x+1, y, z-1}^{(3)}(\mathbf{a} ; \mathbf{c} ; \mathbf{b})\right) \mathrm{M}\left(Q_{x-1, y, z}^{\leftarrow}\left(\mathbf{b} ; \mathbf{c}^{\leftrightarrow} ; \mathbf{a}^{+1}\right)\right) .
\end{aligned}
$$



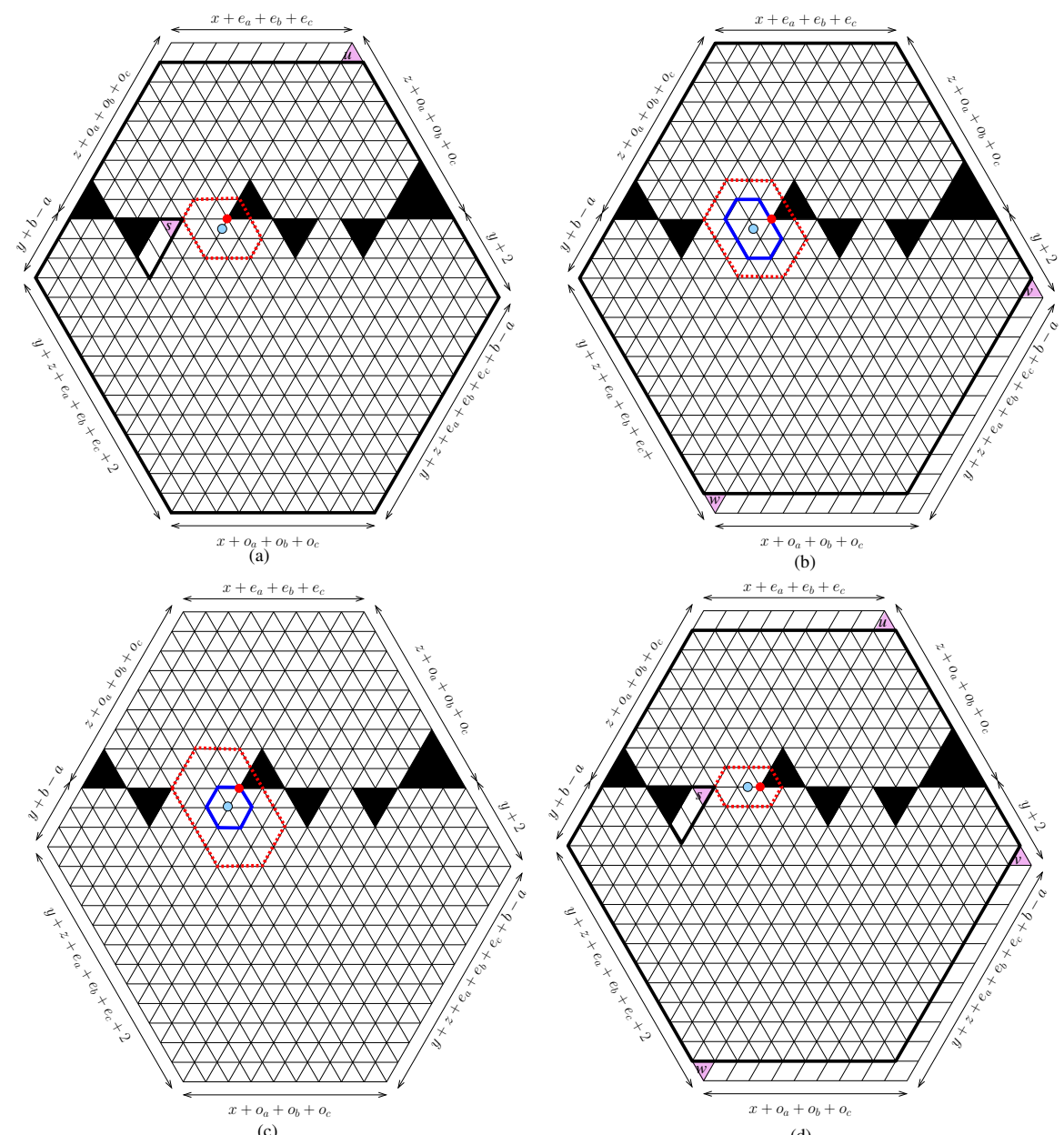

(c)
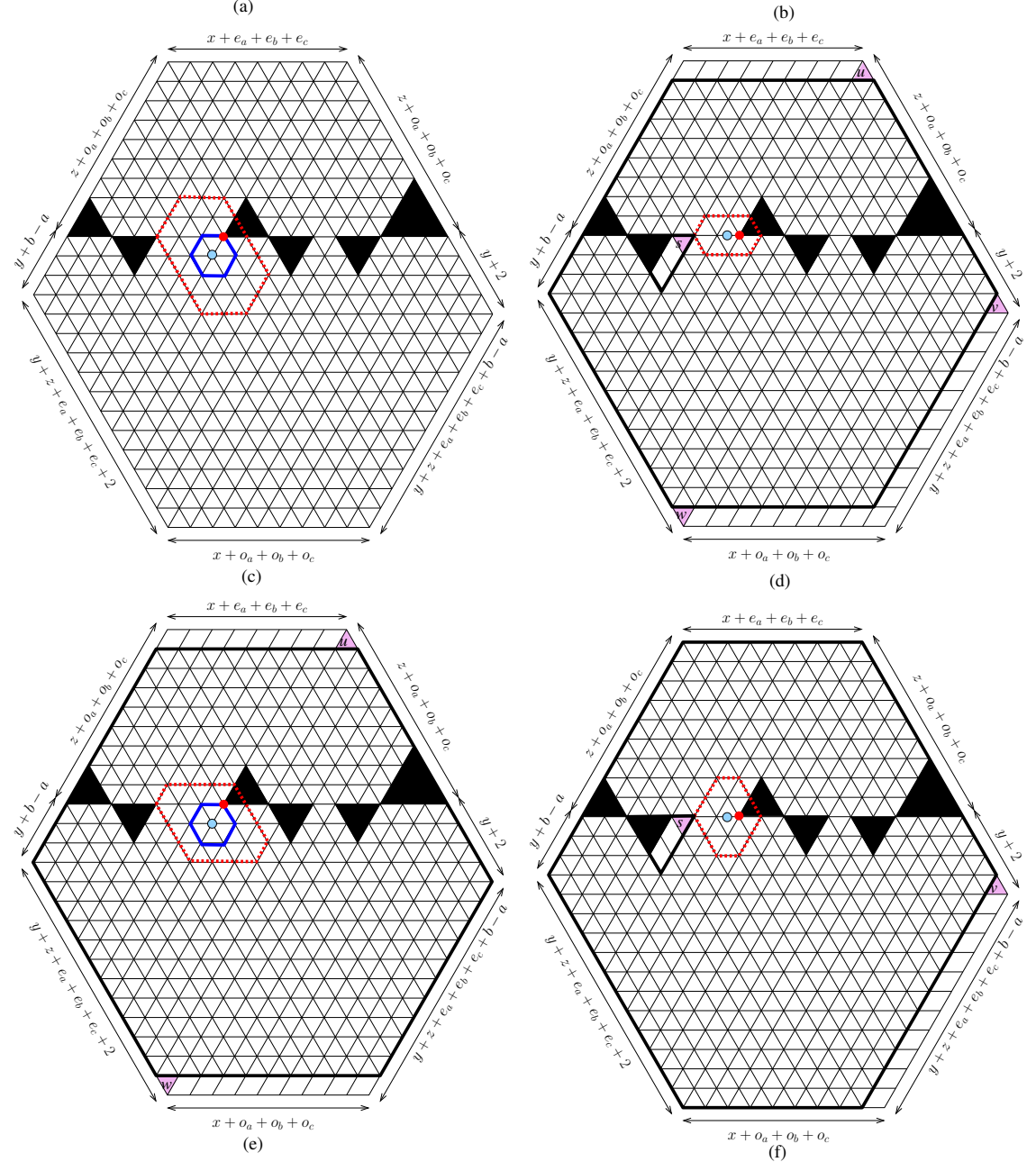

Figure 19: Obtaining a recurrence for $\bar{E}^{(3)}$-type regions with $a \leqslant b$. Kuo condensation is applied to the region $\bar{E}_{2,2,2}^{(3)}(2,2 ; 2,2 ; 3,2)$ (picture (a)) as shown on the picture (b). 


\subsection{Recurrences for $\bar{F}^{(i)}$-type regions}

We apply Kuo's Theorem 47 to the $\bar{F}^{(3)}-, \bar{F}^{(4)}$ - and $\bar{F}^{(5)}$-type regions, as shown in Figures $12(\mathrm{a})$, (b) and (c). The second Kuo's theorem, Theorem 48, is used for the $\bar{F}^{(6)}$-type regions, as in Figure 12(d).

We investigate the case of $\bar{F}^{(6)}$-type regions when $a \geqslant b$. We apply Kuo's Theorem 48 to the dual graph $G$ of the region $\bar{F}_{x, y, z}^{(6)}(\mathbf{a} ; \mathbf{c} ; \mathbf{b})$ with the choice of the four vertices $u, v, w, s$ shown in Figure 20(d). By considering forced lozenges as in Figures $20(\mathrm{a}),(\mathrm{b}),(\mathrm{d}),(\mathrm{e}),(\mathrm{f})$, we get

$$
\begin{gathered}
\mathrm{M}(G-\{u, s\})=\mathrm{M}\left(\bar{G}_{x, y, z-1}^{(5)}\left(\mathbf{a}^{+1} ; \mathbf{c} ; \mathbf{b}\right)\right), \\
\mathrm{M}(G-\{v, w\})=\mathrm{M}\left(\bar{K}_{x, y, z}^{(8)}(\mathbf{a} ; \mathbf{c} ; \mathbf{b})\right), \\
\mathrm{M}(G-\{u, v, w, s\})=\mathrm{M}\left(\bar{E}_{x, y, z-1}^{(1)}\left(\mathbf{b} ; \mathbf{c}^{\leftrightarrow} ; \mathbf{a}^{+1}\right)\right), \\
\mathrm{M}(G-\{u, w\})=\mathrm{M}\left(\bar{F}_{x+1, y, z-1}^{(6)}(\mathbf{a} ; \mathbf{c} ; \mathbf{b})\right), \\
\mathrm{M}(G-\{v, s\})=\mathrm{M}\left(\bar{E}_{x-1, y, z}^{(1)}\left(\mathbf{b} ; \mathbf{c}^{\leftrightarrow} ; \mathbf{a}^{+1}\right)\right) .
\end{gathered}
$$

We note that we reflected the leftover regions by a vertical line in (109) and (111) to get respectively the regions $\bar{E}_{x, y, z-1}^{(1)}\left(\mathbf{b} ; \mathbf{c}^{\leftrightarrow} ; \mathbf{a}^{+1}\right)$ and $\bar{E}_{x-1, y, z}^{(1)}\left(\mathbf{b} ; \mathbf{c}^{\leftrightarrow} ; \mathbf{a}^{+1}\right)$.

Plugging the above 5 equalities into that of Kuo's Theorem 48, we obtain the $\bar{F}^{(6)}$ recurrence for $a \geqslant b$ :

$$
\begin{aligned}
\mathrm{M}\left(\bar{G}_{x, y, z-1}^{(5)}\left(\mathbf{a}^{+1} ; \mathbf{c} ; \mathbf{b}\right)\right) \mathrm{M}( & \left.\bar{K}_{x, y, z}^{(8)}(\mathbf{a} ; \mathbf{c} ; \mathbf{b})\right)= \\
& \mathrm{M}\left(\bar{F}_{x, y, z}^{(6)}(\mathbf{a} ; \mathbf{c} ; \mathbf{b})\right) \mathrm{M}\left(\bar{E}_{x, y, z-1}^{(1)}\left(\mathbf{b} ; \mathbf{c}^{\leftrightarrow} ; \mathbf{a}^{+1}\right)\right) \\
& +\mathrm{M}\left(\bar{F}_{x+1, y, z-1}^{(6)}(\mathbf{a} ; \mathbf{c} ; \mathbf{b})\right) \mathrm{M}\left(\bar{E}_{x-1, y, z}^{(1)}\left(\mathbf{b} ; \mathbf{c}^{\leftrightarrow} ; \mathbf{a}^{+1}\right)\right) .
\end{aligned}
$$

Similar applications of Kuo condensation give the other 6 recurrences:

The $\bar{F}^{(3)}$-recurrence for $a<b$

$$
\begin{aligned}
& \mathrm{M}\left(\bar{F}_{x, y, z}^{(3)}(\mathbf{a} ; \mathbf{c} ; \mathbf{b})\right) \mathrm{M}\left(\bar{E}_{x, y-1, z-1}^{(1)}\left(\mathbf{a} ; \mathbf{c} ; \mathbf{b}^{+1}\right)\right)= \\
& \operatorname{M}\left(\bar{G}_{x, y-1, z-1}^{(2)}\left(\mathbf{a} ; \mathbf{c} ; \mathbf{b}^{+1}\right)\right) \mathrm{M}\left(\bar{K}_{x, y-1, z}^{(8)}(\mathbf{b} ; \mathbf{c} \leftrightarrow ; \mathbf{a})\right) \\
& \quad+\mathrm{M}\left(\bar{F}_{x+1, y, z-1}^{(3)}(\mathbf{a} ; \mathbf{c} ; \mathbf{b})\right) \mathrm{M}\left(\bar{E}_{x-1, y-1, z}^{(1)}\left(\mathbf{a} ; \mathbf{c} ; \mathbf{b}^{+1}\right)\right) .
\end{aligned}
$$

The $\bar{F}^{(3)}$-recurrence for $a \geqslant b$

$$
\mathrm{M}\left(\bar{F}_{x, y, z}^{(3)}(\mathbf{a} ; \mathbf{c} ; \mathbf{b})\right) \mathrm{M}\left(\bar{E}_{x, y, z-1}^{(1)}\left(\mathbf{a} ; \mathbf{c} ; \mathbf{b}^{+1}\right)\right)=
$$




$$
\begin{aligned}
& \mathrm{M}\left(\bar{G}_{x, y, z-1}^{(2)}\left(\mathbf{a} ; \mathbf{c} ; \mathbf{b}^{+1}\right)\right) \mathrm{M}\left(\bar{K}_{x, y-1, z}^{(8)}\left(\mathbf{b} ; \mathbf{c}^{\leftrightarrow} ; \mathbf{a}\right)\right) \\
& \quad+\mathrm{M}\left(\bar{F}_{x+1, y, z-1}^{(3)}(\mathbf{a} ; \mathbf{c} ; \mathbf{b})\right) \mathrm{M}\left(\bar{E}_{x-1, y, z}^{(1)}\left(\mathbf{a} ; \mathbf{c} ; \mathbf{b}^{+1}\right)\right) .
\end{aligned}
$$

The $\bar{F}^{(4)}$-recurrence for $a<b$

$$
\begin{aligned}
\mathrm{M}\left(\bar{F}_{x, y, z}^{(4)}(\mathbf{a} ; \mathbf{c} ; \mathbf{b})\right) \mathrm{M}\left(\bar{K}_{x-1, y, z-1}^{(5)}\left(\mathbf{a}^{+1} ; \mathbf{c} ; \mathbf{b}^{+1}\right)\right)= \\
\mathrm{M}\left(\bar{E}_{x-1, y-1, z}^{(2)}\left(\mathbf{a} ; \mathbf{c} ; \mathbf{b}^{+1}\right)\right) \mathrm{M}\left(\bar{G}_{x, y+1, z-1}^{(2)}\left(\mathbf{a}^{+1} ; \mathbf{c} ; \mathbf{b}\right)\right) \\
+\mathrm{M}\left(\bar{F}_{x-1, y, z-1}^{(4)}\left(\mathbf{a}^{+1} ; \mathbf{c} ; \mathbf{b}^{+1}\right)\right) \mathrm{M}\left(\bar{K}_{x, y, z}^{(5)}(\mathbf{a} ; \mathbf{c} ; \mathbf{b})\right) .
\end{aligned}
$$

The $\bar{F}^{(4)}$-recurrence for $a \geqslant b$

$$
\begin{aligned}
\mathrm{M}\left(\bar{F}_{x, y, z}^{(4)}(\mathbf{a} ; \mathbf{c} ; \mathbf{b})\right) \mathrm{M}\left(\bar{K}_{x-1, y, z-1}^{(5)}\left(\mathbf{a}^{+1} ; \mathbf{c} ; \mathbf{b}^{+1}\right)\right)= \\
\quad \mathrm{M}\left(\bar{E}_{x-1, y, z}^{(2)}\left(\mathbf{a} ; \mathbf{c} ; \mathbf{b}^{+1}\right)\right) \mathrm{M}\left(\bar{G}_{x, y, z-1}^{(2)}\left(\mathbf{a}^{+1} ; \mathbf{c} ; \mathbf{b}\right)\right) \\
+\mathrm{M}\left(\bar{F}_{x-1, y, z-1}^{(4)}\left(\mathbf{a}^{+1} ; \mathbf{c} ; \mathbf{b}^{+1}\right)\right) \mathrm{M}\left(\bar{K}_{x, y, z}^{(5)}(\mathbf{a} ; \mathbf{c} ; \mathbf{b})\right) .
\end{aligned}
$$

The $\bar{F}^{(5)}$-recurrence for $a<b$

$$
\begin{aligned}
\mathrm{M}\left(\bar{F}_{x, y, z}^{(5)}(\mathbf{a} ; \mathbf{c} ; \mathbf{b})\right) \mathrm{M}\left(Q_{x-1, y, z-1}^{\nearrow}\left(\mathbf{a}^{+1} ; \mathbf{c} ; \mathbf{b}^{+1}\right)\right)= \\
\operatorname{M}\left(\bar{E}_{x-1, y-1, z}^{(3)}\left(\mathbf{a} ; \mathbf{c} ; \mathbf{b}^{+1}\right)\right) \mathrm{M}\left(Q_{x, y+1, z-1}^{\nwarrow}\left(\mathbf{a}^{+1} ; \mathbf{c} ; \mathbf{b}\right)\right) \\
+\mathrm{M}\left(\bar{F}_{x-1, y, z-1}^{(5)}\left(\mathbf{a}^{+1} ; \mathbf{c} ; \mathbf{b}^{+1}\right)\right) \mathrm{M}\left(Q_{x, y, z}^{\nearrow}(\mathbf{a} ; \mathbf{c} ; \mathbf{b})\right) .
\end{aligned}
$$

The $\bar{F}^{(5)}$-recurrence for $a \geqslant b$

$$
\begin{aligned}
\mathrm{M}\left(\bar{F}_{x, y, z}^{(5)}(\mathbf{a} ; \mathbf{c} ; \mathbf{b})\right) \mathrm{M}\left(Q_{x-1, y, z-1}^{\nearrow}\left(\mathbf{a}^{+1} ; \mathbf{c} ; \mathbf{b}^{+1}\right)\right)= \\
\operatorname{M}\left(\bar{E}_{x-1, y, z}^{(3)}\left(\mathbf{a} ; \mathbf{c} ; \mathbf{b}^{+1}\right)\right) \mathrm{M}\left(Q_{x, y, z-1}^{\nwarrow}\left(\mathbf{a}^{+1} ; \mathbf{c} ; \mathbf{b}\right)\right) \\
+\mathrm{M}\left(\bar{F}_{x-1, y, z-1}^{(5)}\left(\mathbf{a}^{+1} ; \mathbf{c} ; \mathbf{b}^{+1}\right)\right) \mathrm{M}\left(Q_{x, y, z}^{\nearrow}(\mathbf{a} ; \mathbf{c} ; \mathbf{b})\right) .
\end{aligned}
$$

The $\bar{F}^{(6)}$-recurrence for $a<b$

$$
\begin{aligned}
& \mathrm{M}\left(\bar{G}_{x, y+1, z-1}^{(5)}\left(\mathbf{a}^{+1} ; \mathbf{c} ; \mathbf{b}\right)\right) \mathrm{M}\left(\bar{K}_{x, y, z}^{(8)}(\mathbf{a} ; \mathbf{c} ; \mathbf{b})\right)= \\
& \quad \mathrm{M}\left(\bar{F}_{x, y, z}^{(6)}(\mathbf{a} ; \mathbf{c} ; \mathbf{b})\right) \mathrm{M}\left(\bar{E}_{x, y+1, z-1}^{(1)}\left(\mathbf{b} ; \mathbf{c}^{\leftrightarrow} ; \mathbf{a}^{+1}\right)\right) \\
& \quad+\mathrm{M}\left(\bar{F}_{x+1, y, z-1}^{(6)}(\mathbf{a} ; \mathbf{c} ; \mathbf{b})\right) \mathrm{M}\left(\bar{E}_{x-1, y+1, z}^{(1)}\left(\mathbf{b} ; \mathbf{c}^{\leftrightarrow} ; \mathbf{a}^{+1}\right)\right) .
\end{aligned}
$$




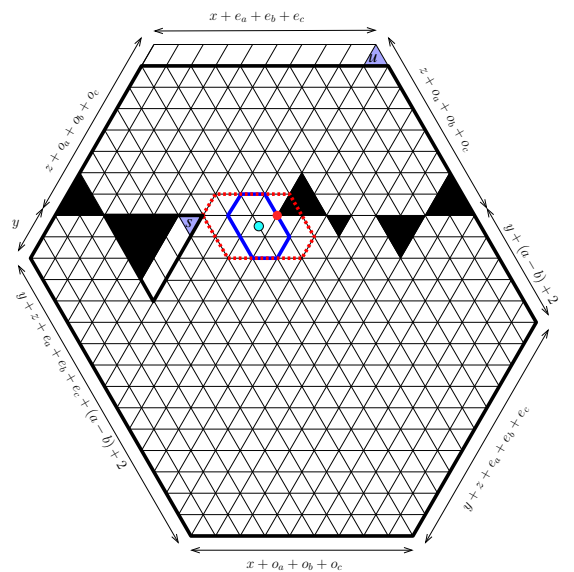

(a)

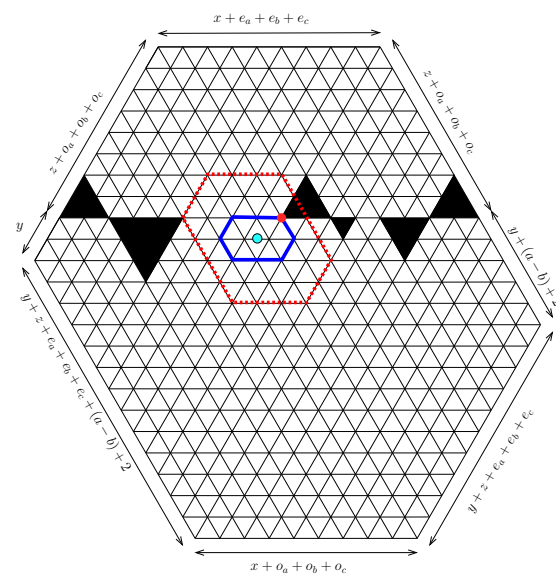

(c)

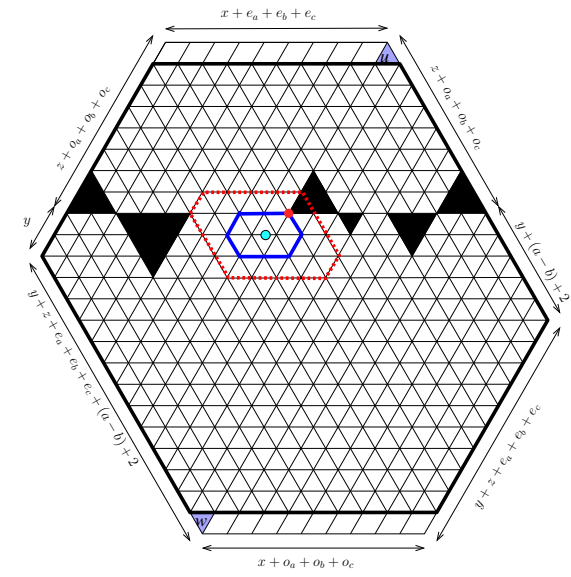

(e)
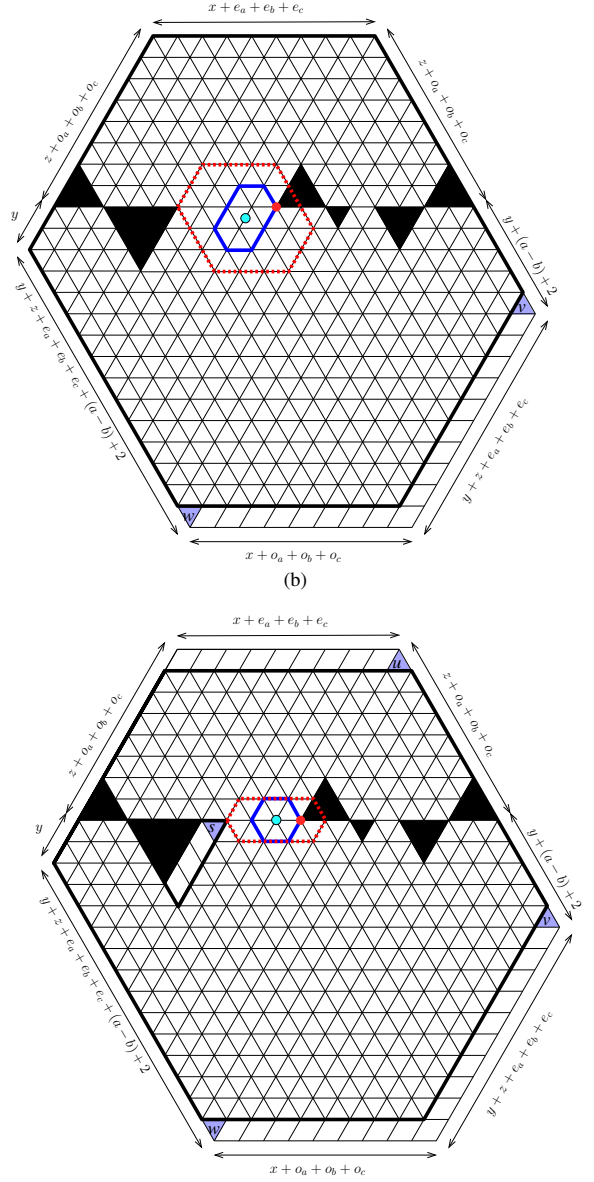

(d)

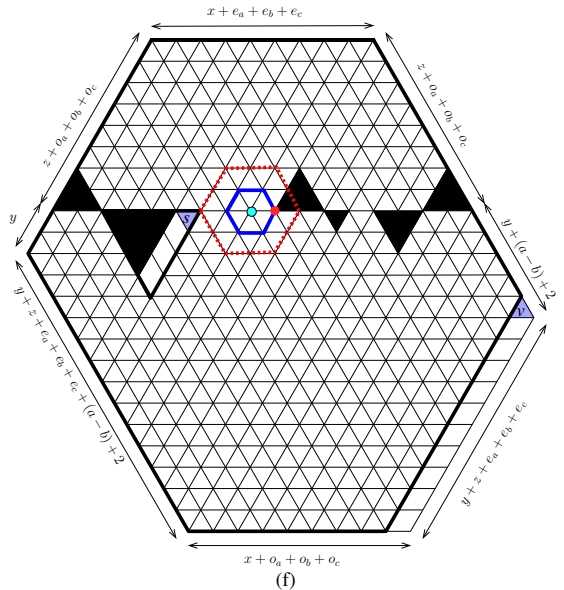

(f)

Figure 20: Obtaining a recurrence for $\bar{F}^{(6)}$-type regions with $a \geqslant b$. Kuo condensation is applied to the region $\bar{F}_{3,2,2}^{(6)}(2,3 ; 2,1 ; 2,2)$ (picture (c)) as shown on the picture (d). 


\subsection{Recurrences for $\bar{G}^{(i)}$-type regions}

We apply Theorem 47 to the $\bar{G}^{(2)}$ - and $\bar{G}^{(3)}$-type regions as in Figures $13(\mathrm{a})$ and (b). For the case of $\bar{G}^{(4)}$ - and $\bar{G}^{(5)}$-type regions, we employ Theorem 48 with the choice of the vertices $u, v, w, s$ shown in Figures 13(c) and (d).

We consider here the case of $\bar{G}^{(3)}$-type regions when $a<b$. We apply Kuo's Theorem 47 to the dual graph $G$ of the region $\bar{G}_{x, y, z}^{(3)}(\mathbf{a} ; \mathbf{c} ; \mathbf{b})$. By considering forced lozenges as in Figures 21(b)-(f), we get

$$
\begin{gathered}
\mathrm{M}(G-\{u, v, w, s\})=\mathrm{M}\left(\bar{E}_{x-1, y, z-1}^{(2)}\left(\mathbf{a}^{+1} ; \mathbf{c} ; \mathbf{b}^{+1}\right)\right), \\
\mathrm{M}(G-\{u, v\})=\mathrm{M}\left(\bar{K}_{x-1, y-1, z}^{(6)}\left(\mathbf{a} ; \mathbf{c} ; \mathbf{b}^{+1}\right)\right), \\
\mathrm{M}(G-\{w, s\})=\mathrm{M}\left(\bar{F}_{x, y+1, z-1}^{(4)}\left(\mathbf{a}^{+1} ; \mathbf{c} ; \mathbf{b}\right)\right), \\
\mathrm{M}(G-\{u, s\})=\mathrm{M}\left(\bar{G}_{x-1, y, z-1}^{(3)}\left(\mathbf{a}^{+1} ; \mathbf{c} ; \mathbf{b}^{+1}\right)\right), \\
\mathrm{M}(G-\{v, w\})=\mathrm{M}\left(\bar{E}_{x, y, z}^{(2)}(\mathbf{a} ; \mathbf{c} ; \mathbf{b})\right) .
\end{gathered}
$$

Plugging the above 5 recurrences into the equation in Kuo's Theorem 47, we obtain the $\bar{G}^{(3)}$-recurrence for $a<b$ :

$$
\begin{aligned}
& \mathrm{M}\left(\bar{G}_{x, y, z}^{(3)}(\mathbf{a} ; \mathbf{c} ; \mathbf{b})\right) \mathrm{M}\left(\bar{E}_{x-1, y, z-1}^{(2)}\left(\mathbf{a}^{+1} ; \mathbf{c} ; \mathbf{b}^{+1}\right)\right)= \\
& \operatorname{M}\left(\bar{K}_{x-1, y-1, z}^{(6)}\left(\mathbf{a} ; \mathbf{c} ; \mathbf{b}^{+1}\right)\right) \mathrm{M}\left(\bar{F}_{x, y+1, z-1}^{(4)}\left(\mathbf{a}^{+1} ; \mathbf{c} ; \mathbf{b}\right)\right) \\
&+\mathrm{M}\left(\bar{G}_{x-1, y, z-1}^{(3)}\left(\mathbf{a}^{+1} ; \mathbf{c} ; \mathbf{b}^{+1}\right)\right) \mathrm{M}\left(\bar{E}_{x, y, z}^{(2)}(\mathbf{a} ; \mathbf{c} ; \mathbf{b})\right) .
\end{aligned}
$$

Similarly, we have the other 7 recurrences:

The $\bar{G}^{(2)}$-recurrence for $a<b$

$$
\begin{aligned}
& \mathrm{M}\left(\bar{G}_{x, y, z}^{(2)}(\mathbf{a} ; \mathbf{c} ; \mathbf{b})\right) \mathrm{M}\left(\bar{E}_{x-1, y, z-1}^{(1)}\left(\mathbf{a}^{+1} ; \mathbf{c} ; \mathbf{b}^{+1}\right)\right)= \\
& \mathrm{M}\left(\bar{K}_{x-1, y-1, z}^{(5)}\left(\mathbf{a} ; \mathbf{c} ; \mathbf{b}^{+1}\right)\right) \mathrm{M}\left(\bar{F}_{x, y+1, z-1}^{(3)}\left(\mathbf{a}^{+1} ; \mathbf{c} ; \mathbf{b}\right)\right) \\
&+\mathrm{M}\left(\bar{G}_{x-1, y, z-1}^{(2)}\left(\mathbf{a}^{+1} ; \mathbf{c} ; \mathbf{b}^{+1}\right)\right) \mathrm{M}\left(\bar{E}_{x, y, z}^{(1)}(\mathbf{a} ; \mathbf{c} ; \mathbf{b})\right) .
\end{aligned}
$$

The $\bar{G}^{(2)}$-recurrence for $a>b$

$$
\begin{aligned}
\mathrm{M}\left(\bar{G}_{x, y, z}^{(2)}(\mathbf{a} ; \mathbf{c} ; \mathbf{b})\right) \mathrm{M}\left(\bar{E}_{x-1, y, z-1}^{(1)}\left(\mathbf{a}^{+1} ; \mathbf{c} ; \mathbf{b}^{+1}\right)\right)= \\
\quad \mathrm{M}\left(\bar{K}_{x-1, y, z}^{(5)}\left(\mathbf{a} ; \mathbf{c} ; \mathbf{b}^{+1}\right)\right) \mathrm{M}\left(\bar{F}_{x, y, z-1}^{(3)}\left(\mathbf{a}^{+1} ; \mathbf{c} ; \mathbf{b}\right)\right) \\
\quad+\mathrm{M}\left(\bar{G}_{x-1, y, z-1}^{(2)}\left(\mathbf{a}^{+1} ; \mathbf{c} ; \mathbf{b}^{+1}\right)\right) \mathrm{M}\left(\bar{E}_{x, y, z}^{(1)}(\mathbf{a} ; \mathbf{c} ; \mathbf{b})\right) .
\end{aligned}
$$


The $\bar{G}^{(2)}$-recurrence for $a=b$

$$
\begin{aligned}
\mathrm{M}\left(\bar{G}_{x, y, z}^{(2)}(\mathbf{a} ; \mathbf{c} ; \mathbf{b})\right) \mathrm{M}\left(\bar{E}_{x-1, y, z-1}^{(1)}\left(\mathbf{a}^{+1} ; \mathbf{c} ; \mathbf{b}^{+1}\right)\right)= \\
\mathrm{M}\left(\bar{K}_{x-1, y-1, z}^{(5)}\left(\mathbf{a} ; \mathbf{c} ; \mathbf{b}^{+1}\right)\right) \mathrm{M}\left(\bar{F}_{x, y, z-1}^{(3)}\left(\mathbf{a}^{+1} ; \mathbf{c} ; \mathbf{b}\right)\right) \\
\quad+\mathrm{M}\left(\bar{G}_{x-1, y, z-1}^{(2)}\left(\mathbf{a}^{+1} ; \mathbf{c} ; \mathbf{b}^{+1}\right)\right) \mathrm{M}\left(\bar{E}_{x, y, z}^{(1)}(\mathbf{a} ; \mathbf{c} ; \mathbf{b})\right) .
\end{aligned}
$$



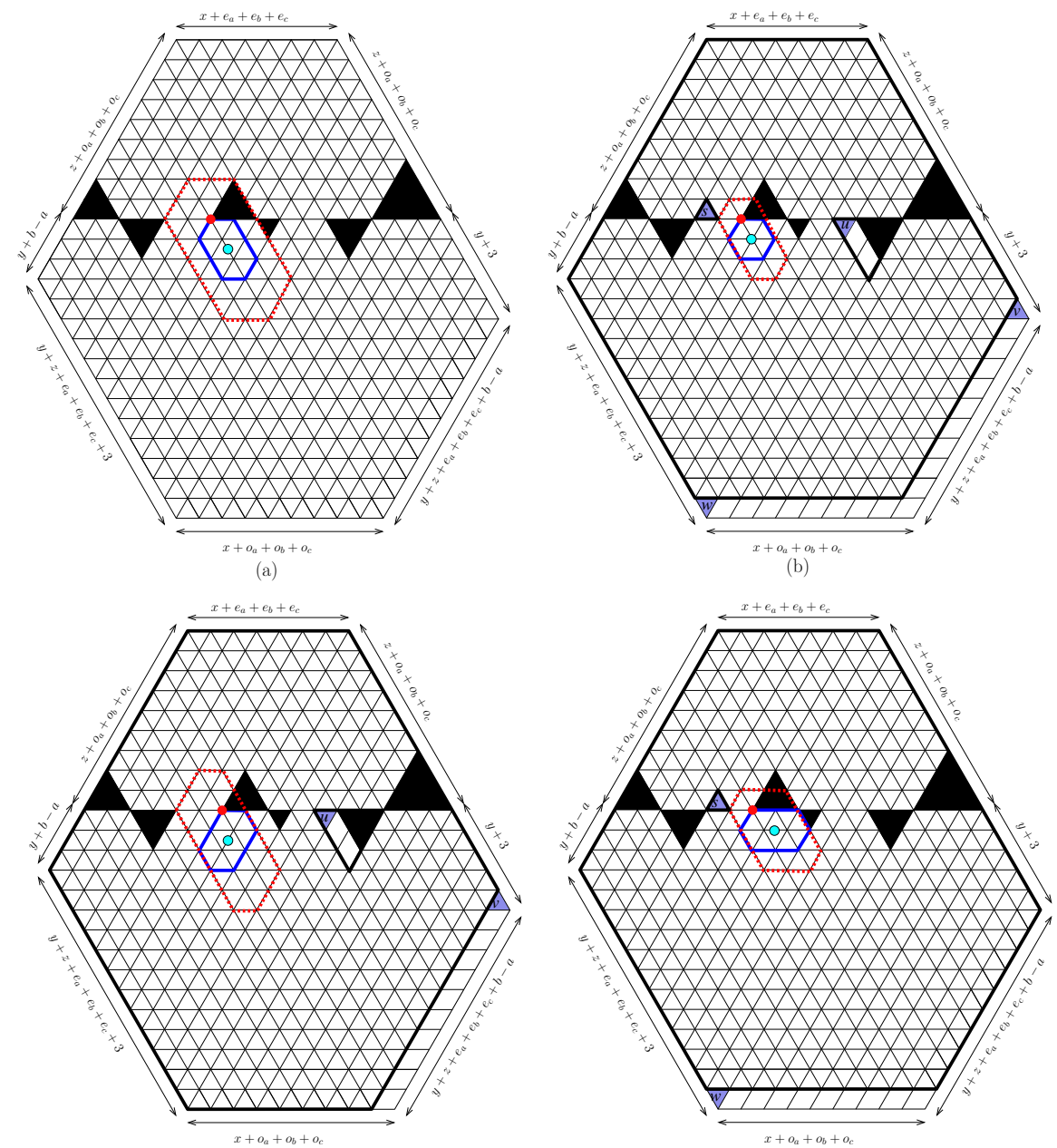

(c)

(d)

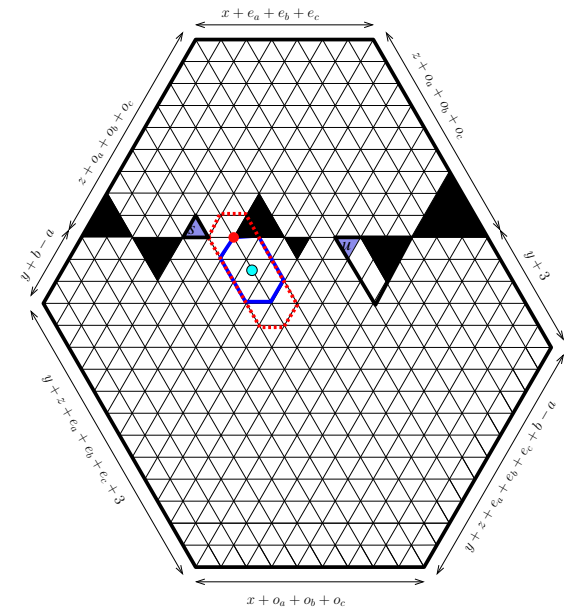

(e)

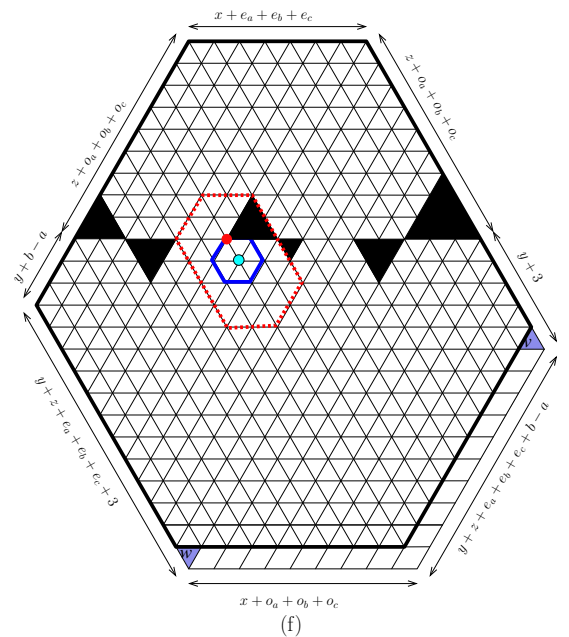

Figure 21: Obtaining a recurrence for $\bar{G}^{(3)}$-type regions with $a<b$. Kuo condensation is applied to the region $\bar{G}_{2,2,2}^{(3)}(2,2 ; 2,1 ; 3,2)$ (picture (a)) as shown on the picture (b). 
The $\bar{G}^{(3)}$-recurrence for $a>b$

$$
\begin{aligned}
\mathrm{M}\left(\bar{G}_{x, y, z}^{(3)}(\mathbf{a} ; \mathbf{c} ; \mathbf{b})\right) \mathrm{M}\left(\bar{E}_{x-1, y, z-1}^{(2)}\left(\mathbf{a}^{+1} ; \mathbf{c} ; \mathbf{b}^{+1}\right)\right)= \\
\operatorname{M}\left(\bar{K}_{x-1, y, z}^{(6)}\left(\mathbf{a} ; \mathbf{c} ; \mathbf{b}^{+1}\right)\right) \mathrm{M}\left(\bar{F}_{x, y, z-1}^{(4)}\left(\mathbf{a}^{+1} ; \mathbf{c} ; \mathbf{b}\right)\right) \\
+\mathrm{M}\left(\bar{G}_{x-1, y, z-1}^{(3)}\left(\mathbf{a}^{+1} ; \mathbf{c} ; \mathbf{b}^{+1}\right)\right) \mathrm{M}\left(\bar{E}_{x, y, z}^{(2)}(\mathbf{a} ; \mathbf{c} ; \mathbf{b})\right) .
\end{aligned}
$$

The $\bar{G}^{(3)}$-recurrence for $a=b$

$$
\begin{aligned}
& \mathrm{M}\left(\bar{G}_{x, y, z}^{(3)}(\mathbf{a} ; \mathbf{c} ; \mathbf{b})\right) \mathrm{M}\left(\bar{E}_{x-1, y, z-1}^{(2)}\left(\mathbf{a}^{+1} ; \mathbf{c} ; \mathbf{b}^{+1}\right)\right)= \\
& \operatorname{M}\left(\bar{K}_{x-1, y-1, z}^{(6)}\left(\mathbf{a} ; \mathbf{c} ; \mathbf{b}^{+1}\right)\right) \mathrm{M}\left(\bar{F}_{x, y, z-1}^{(4)}\left(\mathbf{a}^{+1} ; \mathbf{c} ; \mathbf{b}\right)\right) \\
&+\mathrm{M}\left(\bar{G}_{x-1, y, z-1}^{(3)}\left(\mathbf{a}^{+1} ; \mathbf{c} ; \mathbf{b}^{+1}\right)\right) \mathrm{M}\left(\bar{E}_{x, y, z}^{(2)}(\mathbf{a} ; \mathbf{c} ; \mathbf{b})\right) .
\end{aligned}
$$

The $\bar{G}^{(4)}$-recurrence for $a<b$

$$
\begin{aligned}
& \mathrm{M}\left(\bar{F}_{x, y+1, z-1}^{(5)}\left(\mathbf{a}^{+1} ; \mathbf{c} ; \mathbf{b}\right)\right) \mathrm{M}\left(\bar{E}_{x, y, z}^{(3)}(\mathbf{a} ; \mathbf{c} ; \mathbf{b})\right)= \\
& \operatorname{M}\left(\bar{G}_{x, y, z}^{(4)}(\mathbf{a} ; \mathbf{c} ; \mathbf{b})\right) \operatorname{M}\left(Q_{x, y+1, z-1}^{\nearrow}\left(\mathbf{a}^{+1} ; \mathbf{c} ; \mathbf{b}\right)\right) \\
& +\mathrm{M}\left(\bar{G}_{x+1, y, z-1}^{(4)}(\mathbf{a} ; \mathbf{c} ; \mathbf{b})\right) \mathrm{M}\left(Q_{x-1, y+1, z}^{\nearrow}\left(\mathbf{a}^{+1} ; \mathbf{c} ; \mathbf{b}\right)\right) .
\end{aligned}
$$

The $\bar{G}^{(4)}$-recurrence for $a \geqslant b$

$$
\begin{aligned}
& \mathrm{M}\left(\bar{F}_{x, y, z-1}^{(5)}\left(\mathbf{a}^{+1} ; \mathbf{c} ; \mathbf{b}\right)\right) \mathrm{M}\left(\bar{E}_{x, y, z}^{(3)}(\mathbf{a} ; \mathbf{c} ; \mathbf{b})\right)= \\
& \quad \mathrm{M}\left(\bar{G}_{x, y, z}^{(4)}(\mathbf{a} ; \mathbf{c} ; \mathbf{b})\right) \mathrm{M}\left(Q_{x, y, z-1}^{\nearrow}\left(\mathbf{a}^{+1} ; \mathbf{c} ; \mathbf{b}\right)\right) \\
& \quad+\mathrm{M}\left(\bar{G}_{x+1, y, z-1}^{(4)}(\mathbf{a} ; \mathbf{c} ; \mathbf{b})\right) \mathrm{M}\left(Q_{x-1, y, z}^{\nearrow}\left(\mathbf{a}^{+1} ; \mathbf{c} ; \mathbf{b}\right)\right) .
\end{aligned}
$$

The $\bar{G}^{(5)}$-recurrence for $a<b$

$$
\begin{aligned}
& \mathrm{M}\left(Q_{x, y+1, z-1}^{\leftarrow}\left(\mathbf{b} ; \mathbf{c}^{\leftrightarrow} ; \mathbf{a}^{+1}\right)\right) \mathrm{M}\left(\bar{E}_{x, y, z}^{(1)}\left(\mathbf{b} ; \mathbf{c}^{\leftrightarrow} ; \mathbf{a}\right)\right)= \\
& \quad \mathrm{M}\left(\bar{G}_{x, y, z}^{(5)}(\mathbf{a} ; \mathbf{c} ; \mathbf{b})\right) \mathrm{M}\left(\bar{K}_{x, y, z-1}^{(5)}\left(\mathbf{b} ; \mathbf{c}^{\leftrightarrow} ; \mathbf{a}^{+1}\right)\right) \\
& \quad+\mathrm{M}\left(\bar{G}_{x+1, y, z-1}^{(5)}(\mathbf{a} ; \mathbf{c} ; \mathbf{b})\right) \mathrm{M}\left(\bar{K}_{x-1, y, z}^{(5)}\left(\mathbf{b} ; \mathbf{c}^{\leftrightarrow} ; \mathbf{a}^{+1}\right)\right)
\end{aligned}
$$

The $\bar{G}^{(5)}$-recurrence for $a \geqslant b$

$$
\begin{aligned}
& \mathrm{M}\left(Q_{x, y, z-1}^{\leftarrow}\left(\mathbf{b} ; \mathbf{c}^{\leftrightarrow} ; \mathbf{a}^{+1}\right)\right) \mathrm{M}\left(\bar{E}_{x, y, z}^{(1)}\left(\mathbf{b} ; \mathbf{c}^{\leftrightarrow} ; \mathbf{a}\right)\right)= \\
& \quad \operatorname{M}\left(\bar{G}_{x, y, z}^{(5)}(\mathbf{a} ; \mathbf{c} ; \mathbf{b})\right) \mathrm{M}\left(\bar{K}_{x, y, z-1}^{(5)}\left(\mathbf{b} ; \mathbf{c}^{\leftrightarrow} ; \mathbf{a}^{+1}\right)\right) \\
& \quad+\mathrm{M}\left(\bar{G}_{x+1, y, z-1}^{(5)}(\mathbf{a} ; \mathbf{c} ; \mathbf{b})\right) \mathrm{M}\left(\bar{K}_{x-1, y-1, z}^{(5)}\left(\mathbf{b} ; \mathbf{c}^{\leftrightarrow} ; \mathbf{a}^{+1}\right)\right) .
\end{aligned}
$$




\subsection{Recurrences for $\bar{K}^{(i)}$-type regions}

For the final groups of regions, $\bar{K}^{(5)}-\bar{K}^{(8)}$, we apply Kuo condensation in the same way as we did for the $\bar{G}^{(i)}$-type regions above. The placements of the $u$-, $v$-, w- and $s$ - triangles are shown particularly in Figure 14.

We will demonstrate here the case of $\bar{K}^{(8)}$-type regions when $a \geqslant b$. We apply Kuo's Theorem 48 to the dual graph $G$ of the region $\bar{K}_{x, y, z}^{(8)}(\mathbf{a} ; \mathbf{c} ; \mathbf{b})$. By considering forced lozenges as in Figures 22(a),(b),(d),(e),(f), we get respectively

$$
\begin{gathered}
\mathrm{M}(G-\{u, s\})=\mathrm{M}\left(\bar{E}_{x, y, z-1}^{(1)}\left(\mathbf{b} ; \mathbf{c}^{\leftrightarrow} ; \mathbf{a}^{+1}\right)\right), \\
\mathrm{M}(G-\{v, w\})=\mathrm{M}\left(\bar{F}_{x, y, z}^{(3)}\left(\mathbf{b} ; \mathbf{c}^{\leftrightarrow} ; \mathbf{a}\right)\right), \\
\mathrm{M}(G-\{u, v, w, s\})=\mathrm{M}\left(\bar{G}_{x, y-1, z-1}^{(2)}\left(\mathbf{b} ; \mathbf{c}^{\leftrightarrow} ; \mathbf{a}^{+1}\right)\right), \\
\mathrm{M}(G-\{u, w\})=\mathrm{M}\left(\bar{K}_{x+1, y, z-1}^{(8)}(\mathbf{a} ; \mathbf{c} ; \mathbf{b})\right), \\
\mathrm{M}(G-\{v, s\})=\mathrm{M}\left(\bar{G}_{x-1, y-1, z}^{(2)}\left(\mathbf{b} ; \mathbf{c}^{\leftrightarrow} ; \mathbf{a}^{+1}\right) .\right.
\end{gathered}
$$

One could realize that we reflected the leftover regions by a vertical line in (135), (136), (137) and (139).

Plugging the above 5 equalities into that of Kuo's Theorem 48, we have the $\bar{K}^{(8)}$ recurrence for $a \geqslant b$

$$
\begin{aligned}
& \mathrm{M}\left(\bar{E}_{x, y, z-1}^{(1)}\left(\mathbf{b} ; \mathbf{c}^{\leftrightarrow} ; \mathbf{a}^{+1}\right)\right) \mathrm{M}\left(\bar{F}_{x, y, z}^{(3)}\left(\mathbf{b} ; \mathbf{c}^{\leftrightarrow} ; \mathbf{a}\right)\right)= \\
& \quad \mathrm{M}\left(\bar{K}_{x, y, z}^{(8)}(\mathbf{a} ; \mathbf{c} ; \mathbf{b})\right) \mathrm{M}\left(\bar{G}_{x, y-1, z-1}^{(2)}\left(\mathbf{b} ; \mathbf{c}^{\leftrightarrow} ; \mathbf{a}^{+1}\right)\right) \\
& \quad+\mathrm{M}\left(\bar{K}_{x+1, y, z-1}^{(8)}(\mathbf{a} ; \mathbf{c} ; \mathbf{b})\right) \mathrm{M}\left(\bar{G}_{x-1, y-1, z}^{(2)}\left(\mathbf{b} ; \mathbf{c}^{\leftrightarrow} ; \mathbf{a}^{+1}\right)\right) .
\end{aligned}
$$

We also have 7 more recurrences by similar applications of Kuo condensation: The $\bar{K}^{(5)}$-recurrence for $a<b$

$$
\begin{aligned}
\mathrm{M}\left(\bar{K}_{x, y, z}^{(5)}(\mathbf{a} ; \mathbf{c} ; \mathbf{b})\right) \mathrm{M}\left(Q_{x-1, y, z-1}^{\leftarrow}\left(\mathbf{a}^{+1} ; \mathbf{c} ; \mathbf{b}^{+1}\right)\right)= \\
\quad \mathrm{M}\left(Q_{x-1, y-1, z}^{\nwarrow}\left(\mathbf{a} ; \mathbf{c} ; \mathbf{b}^{+1}\right)\right) \mathrm{M}\left(\bar{E}_{x, y+1, z-1}^{(1)}\left(\mathbf{a}^{+1} ; \mathbf{c} ; \mathbf{b}\right)\right) \\
\quad+\mathrm{M}\left(\bar{K}_{x-1, y, z-1}^{(5)}\left(\mathbf{a}^{+1} ; \mathbf{c} ; \mathbf{b}^{+1}\right)\right) \mathrm{M}\left(Q_{x, y, z}^{\leftarrow}(\mathbf{a} ; \mathbf{c} ; \mathbf{b})\right)
\end{aligned}
$$

The $\bar{K}^{(5)}$-recurrence for $a \geqslant b$

$$
\mathrm{M}\left(\bar{K}_{x, y, z}^{(5)}(\mathbf{a} ; \mathbf{c} ; \mathbf{b})\right) \mathrm{M}\left(Q_{x-1, y, z-1}^{\leftarrow}\left(\mathbf{a}^{+1} ; \mathbf{c} ; \mathbf{b}^{+1}\right)\right)=
$$




$$
\begin{aligned}
& \mathrm{M}\left(Q_{x-1, y, z}^{\nwarrow}\left(\mathbf{a} ; \mathbf{c} ; \mathbf{b}^{+1}\right)\right) \mathrm{M}\left(\bar{E}_{x, y, z-1}^{(1)}\left(\mathbf{a}^{+1} ; \mathbf{c} ; \mathbf{b}\right)\right) \\
& \quad+\mathrm{M}\left(\bar{K}_{x-1, y, z-1}^{(5)}\left(\mathbf{a}^{+1} ; \mathbf{c} ; \mathbf{b}^{+1}\right)\right) \mathrm{M}\left(Q_{x, y, z}^{\leftarrow}(\mathbf{a} ; \mathbf{c} ; \mathbf{b})\right) .
\end{aligned}
$$

The $\bar{K}^{(6)}$-recurrence for $a<b$

$$
\begin{aligned}
\mathrm{M}\left(\bar{K}_{x, y, z}^{(6)}(\mathbf{a} ; \mathbf{c} ; \mathbf{b})\right) \mathrm{M}\left(\bar{F}_{x-1, y, z-1}^{(5)}\left(\mathbf{a}^{+1} ; \mathbf{c} ; \mathbf{b}^{+1}\right)\right)= \\
\operatorname{M}\left(\bar{G}_{x-1, y-1, z}^{(4)}\left(\mathbf{a} ; \mathbf{c} ; \mathbf{b}^{+1}\right)\right) \mathrm{M}\left(\bar{E}_{x, y+1, z-1}^{(2)}\left(\mathbf{a}^{+1} ; \mathbf{c} ; \mathbf{b}\right)\right) \\
+\mathrm{M}\left(\bar{K}_{x-1, y, z-1}^{(6)}\left(\mathbf{a}^{+1} ; \mathbf{c} ; \mathbf{b}^{+1}\right)\right) \mathrm{M}\left(\bar{F}_{x, y, z}^{(5)}(\mathbf{a} ; \mathbf{c} ; \mathbf{b})\right) .
\end{aligned}
$$

The $\bar{K}^{(6)}$-recurrence for $a \geqslant b$

$$
\begin{aligned}
\mathrm{M}\left(\bar{K}_{x, y, z}^{(6)}(\mathbf{a} ; \mathbf{c} ; \mathbf{b})\right) \mathrm{M}\left(\bar{F}_{x-1, y, z-1}^{(5)}\left(\mathbf{a}^{+1} ; \mathbf{c} ; \mathbf{b}^{+1}\right)\right)= \\
\quad \mathrm{M}\left(\bar{G}_{x-1, y, z}^{(4)}\left(\mathbf{a} ; \mathbf{c} ; \mathbf{b}^{+1}\right)\right) \mathrm{M}\left(\bar{E}_{x, y, z-1}^{(2)}\left(\mathbf{a}^{+1} ; \mathbf{c} ; \mathbf{b}\right)\right) \\
+\mathrm{M}\left(\bar{K}_{x-1, y, z-1}^{(6)}\left(\mathbf{a}^{+1} ; \mathbf{c} ; \mathbf{b}^{+1}\right)\right) \mathrm{M}\left(\bar{F}_{x, y, z}^{(5)}(\mathbf{a} ; \mathbf{c} ; \mathbf{b})\right) .
\end{aligned}
$$

The $\bar{K}^{(7)}$-recurrence for $a<b$

$$
\begin{aligned}
& \mathrm{M}\left(\bar{E}_{x, y+1, z-1}^{(3)}\left(\mathbf{a}^{+1} ; \mathbf{c} ; \mathbf{b}\right)\right) \mathrm{M}\left(\bar{F}_{x, y, z}^{(6)}(\mathbf{a} ; \mathbf{c} ; \mathbf{b})\right)= \\
& \quad \mathrm{M}\left(\bar{K}_{x, y, z}^{(7)}(\mathbf{a} ; \mathbf{c} ; \mathbf{b})\right) \mathrm{M}\left(\bar{G}_{x, y+1, z-1}^{(5)}\left(\mathbf{a}^{+1} ; \mathbf{c} ; \mathbf{b}\right)\right) \\
& +\mathrm{M}\left(\bar{K}_{x+1, y, z-1}^{(7)}(\mathbf{a} ; \mathbf{c} ; \mathbf{b})\right) \mathrm{M}\left(\bar{G}_{x-1, y+1, z}^{(5)}\left(\mathbf{a}^{+1} ; \mathbf{c} ; \mathbf{b}\right)\right) .
\end{aligned}
$$

The $\bar{K}^{(7)}$-recurrence for $a \geqslant b$

$$
\begin{aligned}
& \mathrm{M}\left(\bar{E}_{x, y, z-1}^{(3)}\left(\mathbf{a}^{+1} ; \mathbf{c} ; \mathbf{b}\right)\right) \mathrm{M}\left(\bar{F}_{x, y, z}^{(6)}(\mathbf{a} ; \mathbf{c} ; \mathbf{b})\right)= \\
& \quad \mathrm{M}\left(\bar{K}_{x, y, z}^{(7)}(\mathbf{a} ; \mathbf{c} ; \mathbf{b})\right) \mathrm{M}\left(\bar{G}_{x, y, z-1}^{(5)}\left(\mathbf{a}^{+1} ; \mathbf{c} ; \mathbf{b}\right)\right) \\
& \quad+\mathrm{M}\left(\bar{K}_{x+1, y, z-1}^{(7)}(\mathbf{a} ; \mathbf{c} ; \mathbf{b})\right) \mathrm{M}\left(\bar{G}_{x-1, y, z}^{(5)}\left(\mathbf{a}^{+1} ; \mathbf{c} ; \mathbf{b}\right)\right) .
\end{aligned}
$$

The $\bar{K}^{(8)}$-recurrence for $a<b$

$$
\begin{aligned}
& \mathrm{M}\left(\bar{E}_{x, y+1, z-1}^{(1)}\left(\mathbf{b} ; \mathbf{c}^{\leftrightarrow} ; \mathbf{a}^{+1}\right)\right) \mathrm{M}\left(\bar{F}_{x, y, z}^{(3)}\left(\mathbf{b} ; \mathbf{c}^{\leftrightarrow} ; \mathbf{a}\right)\right)= \\
& \quad \mathrm{M}\left(\bar{K}_{x, y, z}^{(8)}(\mathbf{a} ; \mathbf{c} ; \mathbf{b})\right) \mathrm{M}\left(\bar{G}_{x, y, z-1}^{(2)}\left(\mathbf{b} ; \mathbf{c}^{\leftrightarrow} ; \mathbf{a}^{+1}\right)\right) \\
& \quad+\mathrm{M}\left(\bar{K}_{x+1, y, z-1}^{(8)}(\mathbf{a} ; \mathbf{c} ; \mathbf{b})\right) \mathrm{M}\left(\bar{G}_{x-1, y, z}^{(2)}\left(\mathbf{b} ; \mathbf{c}^{\leftrightarrow} ; \mathbf{a}^{+1}\right)\right) .
\end{aligned}
$$




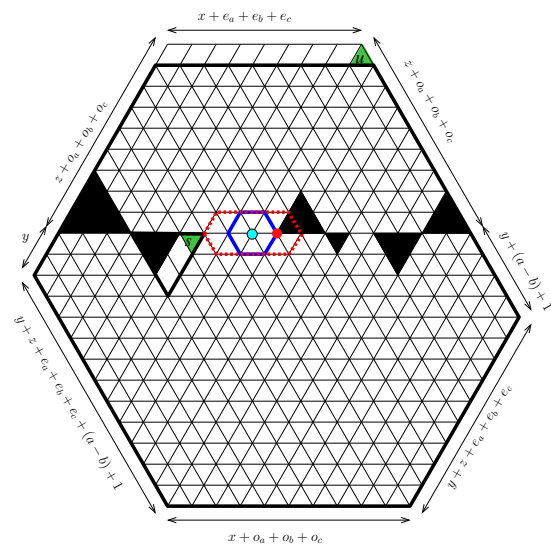

(a)

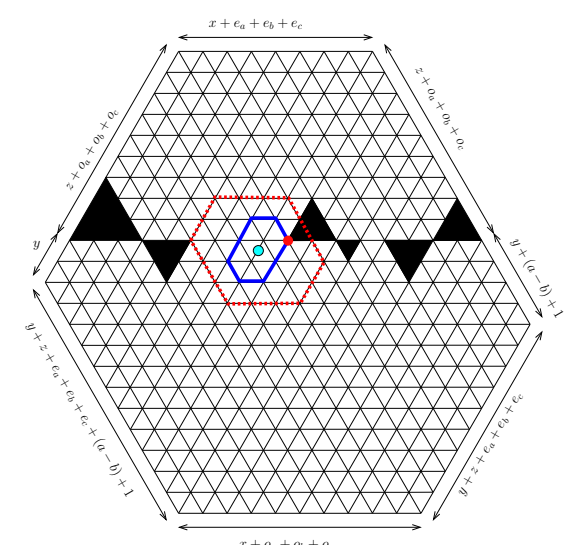

(c)

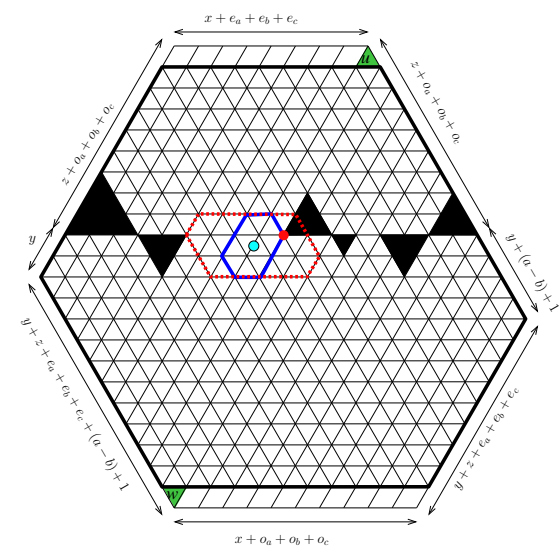

(e)

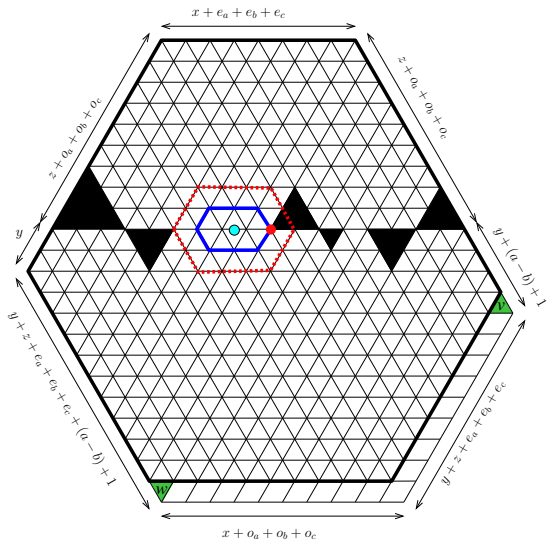

(b)
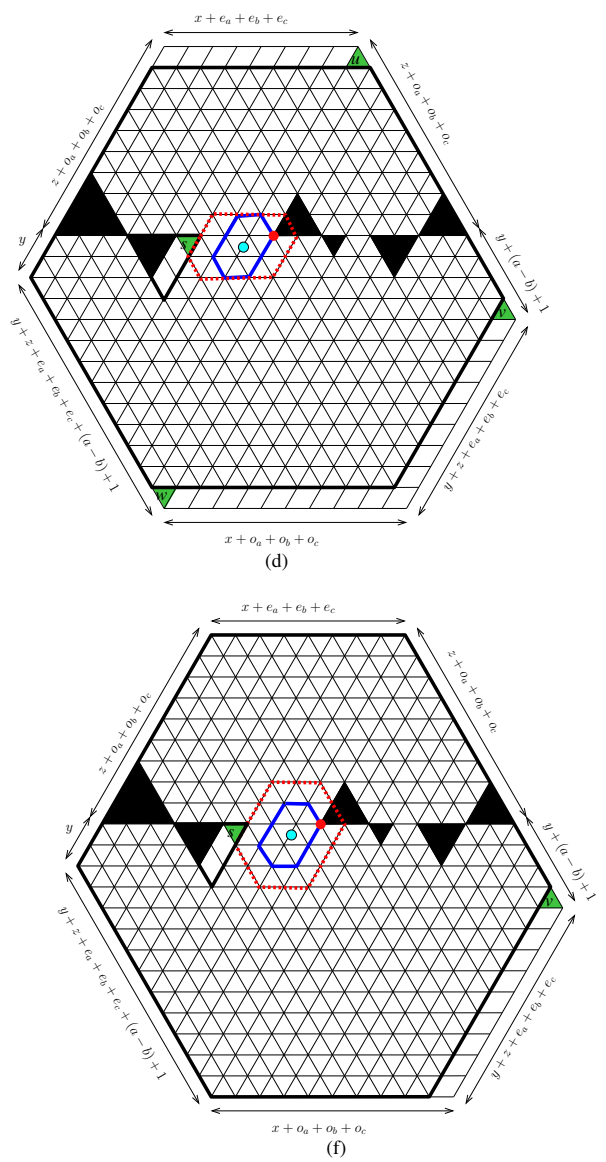

Figure 22: Obtaining a recurrence for $\bar{K}^{(8)}$-type regions with $a \geqslant b$. Kuo condensation is applied to the region $\bar{K}_{3,2,2}^{(8)}(3,2 ; 2,1 ; 2,2)$ (picture (c)) as shown on the picture (d). 


\subsection{Two extremal cases for off-central regions}

This subsection is devoted to two special cases where specific parameters in the off-central regions achieve minimal values. Our first case is when some of the triangles in our ferns have side-length 0.

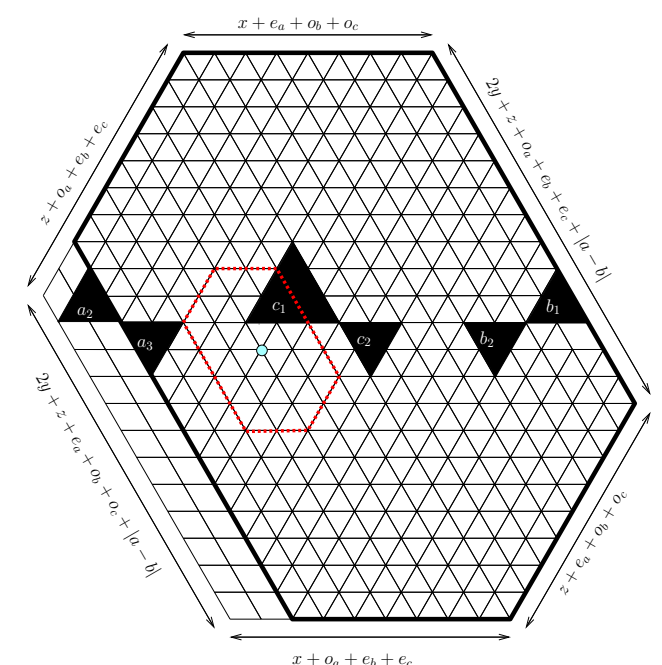

(a)

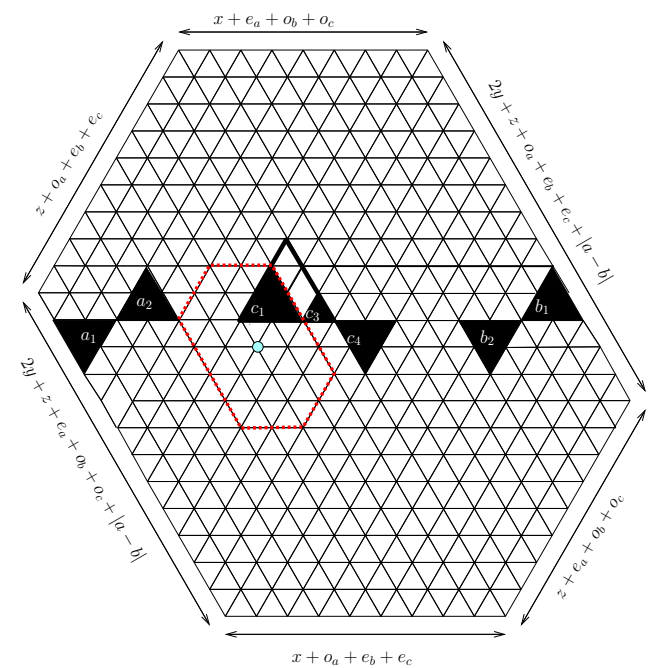

(b)

Figure 23: Eliminating triangles of side-length 0 from the ferns.

Lemma 49. For any off-central region, we can find a new region of the same type (1) whose number of tilings is the same, (2) whose h-parameter is at most that of the original region, (3) whose left and right ferns consist of triangles with positive side-lengths, and (4) whose middle fern contains at most one possible triangle of side length 0 at the beginning.

Proof. The proof is essentially the same as that of Lemma 18 in [31]; however, to make our paper more self-contained, we present the full proof here. We only consider the $R:=E_{x, y, z}^{(2)}(\mathbf{a} ; \mathbf{c} ; \mathbf{b})$ region, and the other regions can be treated similarly.

We will show how to eliminate 0-triangles from the ferns without changing the tiling number or increasing the $h$-parameter. We consider the following three 0 -eliminating procedures for the left fern:

(1) If $a_{1}=a_{2}=\ldots=a_{2 i}=0$, for some $i \geqslant 1$, we can simply truncate the first $2 i$ zero terms in the sequence $\mathbf{a}$. The new region is congruent with the old one; however, strictly speaking, it has fewer 0-triangles in the left fern.

(2) If $a_{1}=0$ and $a_{2}>0$, then we can remove forced lozenges along the southwest side of the region $R$ and obtain the region $E_{x, y, z}^{(2)}\left(a_{3}, \ldots, a_{m} ; \mathbf{c} ; \mathbf{b}\right)$ (see Figure $23(\mathrm{a})$ ). The new region has the same number of tilings as the original one, the $h$-parameter $a_{1}$-unit less than $h$, and fewer 0 -triangles in the left fern.

(3) If $a_{i}=0$, for some $i>1$, then we can eliminate this 0 -triangle by combining the $(i-1)$-th and the $(i+1)$-th triangles in the fern (as shown in Figure 23(b)). 
Repeating these three procedures if needed, one can eliminate all 0-triangles from the left fern. Working similarly for the right fern, we obtain a region with no 0-triangle in the left and right ferns. For the middle fern, we apply procedure (3) to eliminate all 0-triangles, except for a possible 0-triangle at the beginning. This finishes our proof.

In the second special case, we deal with off-central regions whose $y$-parameter achieves its minimal value.

Lemma 50. For any off-central region $R$ with the $y$-parameter minimal, we can find another off-central region whose number of tilings is the same as that of $R$ and whose $h$-parameter is strictly smaller than that of $R$.

Proof. By Lemma 49, we can assume that $a_{i}{ }^{\prime}$ s, $b_{j}{ }^{\prime}$ s, $c_{t}$ 's are all positive, for $i \geqslant 1, j \geqslant 1$, $t \geqslant 2$.

Recall that if $R$ has the root of its middle fern $d$ unit above (resp., below) the center of the auxiliary hexagon $H_{0}$, for $d=0, \frac{1}{2}, 1, \frac{3}{2}$, then $y \geqslant \max (a-b,-2 d)$ (resp., $y \geqslant$ $\max (b-a,-2 d))$. We call these regions upper regions (resp., lower regions). We note that all $\bar{E}^{(i)}$ 's, $\bar{F}^{(i)}$ s, $\bar{G}^{(i)}$ 's, and $\bar{K}^{(i)}$ 's regions are upper ones. There are six cases to distinguish:

Case 1a: $R$ is an upper region, and $a \geqslant b$.

We consider the case when $R$ has the root of the middle fern $d$-unit above the center of the auxiliary hexagon, for $d=1 / 2,1,3 / 2$. If $a \geqslant b$, then $y \geqslant 0$, we can find a 'bar' or 'un-bar' counterpart of the region with the same number of tilings and the $h$-parameter strictly smaller. In particular, by considering forced lozenges, we get

$$
\begin{aligned}
& \mathrm{M}\left(E_{x, 0, z}^{(i)}(\mathbf{a} ; \mathbf{c} ; \mathbf{b})\right)=\mathrm{M}\left(\bar{E}_{x, \min \left(a_{1}, a-b\right), z}^{(i)}\left(a_{2}, \ldots, a_{m} ; \mathbf{c} ; \mathbf{b}\right)\right), \\
& \mathrm{M}\left(F_{x, 0, z}^{(j)}(\mathbf{a} ; \mathbf{c} ; \mathbf{b})\right)=\mathrm{M}\left(\bar{F}_{x, \min \left(a_{1}, a-b\right), z}^{(j)}\left(a_{2}, \ldots, a_{m} ; \mathbf{c} ; \mathbf{b}\right)\right), \\
& \mathrm{M}\left(G_{x, 0, z}^{(k)}(\mathbf{a} ; \mathbf{c} ; \mathbf{b})\right)=\mathrm{M}\left(\bar{G}_{x, \min \left(a_{1}, a-b\right), z}^{(k)}\left(a_{2}, \ldots, a_{m} ; \mathbf{c} ; \mathbf{b}\right)\right), \\
& \mathrm{M}\left(K_{x, 0, z}^{(l)}(\mathbf{a} ; \mathbf{c} ; \mathbf{b})\right)=\mathrm{M}\left(\bar{K}_{x, \min \left(a_{1}, a-b\right), z}^{(l)}\left(a_{2}, \ldots, a_{m} ; \mathbf{c} ; \mathbf{b}\right)\right),
\end{aligned}
$$

for $i=1,2,3,4, j=3,4,5,6, k=2,3,4,5$, and $l=5,6,7,8$ (see Figure 24 for examples). Similarly, we get

$$
\begin{aligned}
& \mathrm{M}\left(\bar{E}_{x, 0, z}^{(i)}(\mathbf{a} ; \mathbf{c} ; \mathbf{b})\right)=\mathrm{M}\left(E_{x, \min \left(a_{1}, a-b\right), z}^{(i)}\left(a_{2}, \ldots, a_{m} ; \mathbf{c} ; \mathbf{b}\right)\right), \\
& \mathrm{M}\left(\bar{F}_{x, 0, z}^{(j)}(\mathbf{a} ; \mathbf{c} ; \mathbf{b})\right)=\mathrm{M}\left(F_{x, \min \left(a_{1}, a-b\right), z}^{(j)}\left(a_{2}, \ldots, a_{m} ; \mathbf{c} ; \mathbf{b}\right)\right), \\
& \mathrm{M}\left(\bar{G}_{x, 0, z}^{(k)}(\mathbf{a} ; \mathbf{c} ; \mathbf{b})\right)=\mathrm{M}\left(G_{x, \min \left(a_{1}, a-b\right), z}^{(k)}\left(a_{2}, \ldots, a_{m} ; \mathbf{c} ; \mathbf{b}\right)\right), \\
& \mathrm{M}\left(\bar{K}_{x, 0, z}^{(l)}(\mathbf{a} ; \mathbf{c} ; \mathbf{b})\right)=\mathrm{M}\left(K_{x, \min \left(a_{1}, a-b\right), z}^{(l)}\left(a_{2}, \ldots, a_{m} ; \mathbf{c} ; \mathbf{b}\right)\right),
\end{aligned}
$$

for $i=1,2,3,4, j=3,4,5,6, k=2,3,4,5$, and $l=5,6,7,8$ (see Figure 25 for examples). 


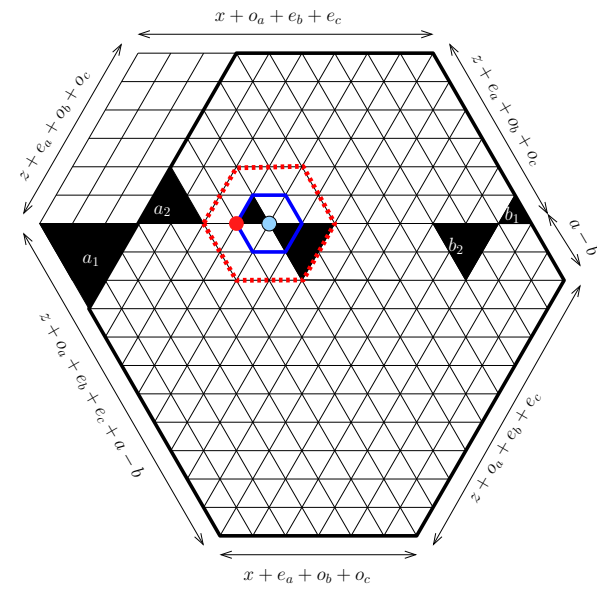

(a)

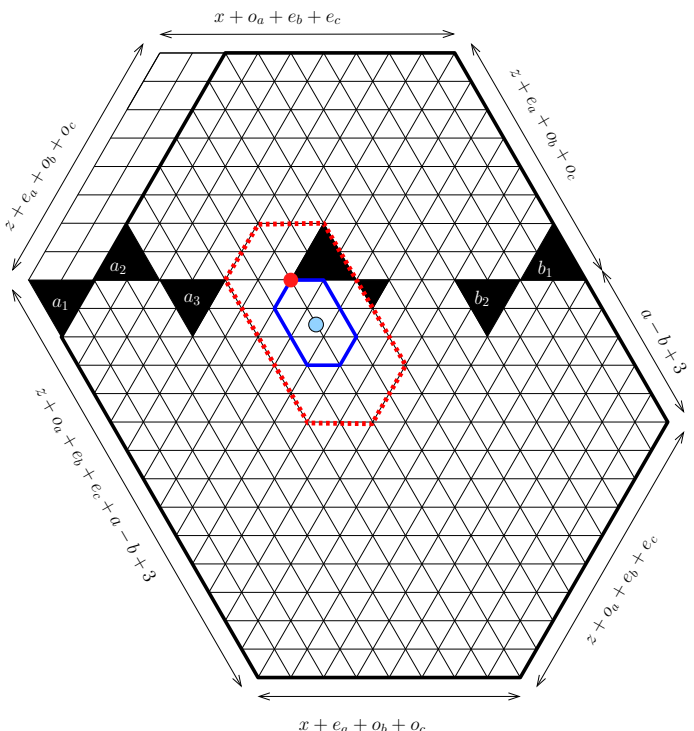

(b)

Figure 24: (a) Obtaining an $\bar{E}^{(1)}$-type region from the region $E_{x, 0, z}^{(1)}(\mathbf{a} ; \mathbf{c} ; \mathbf{b})$ with $a \geqslant b$. (b) Obtaining an $\bar{G}^{(3)}$-type region from the region $G_{x, 0, z}^{(3)}(\mathbf{a} ; \mathbf{c} ; \mathbf{b})$ with $a \geqslant b$.

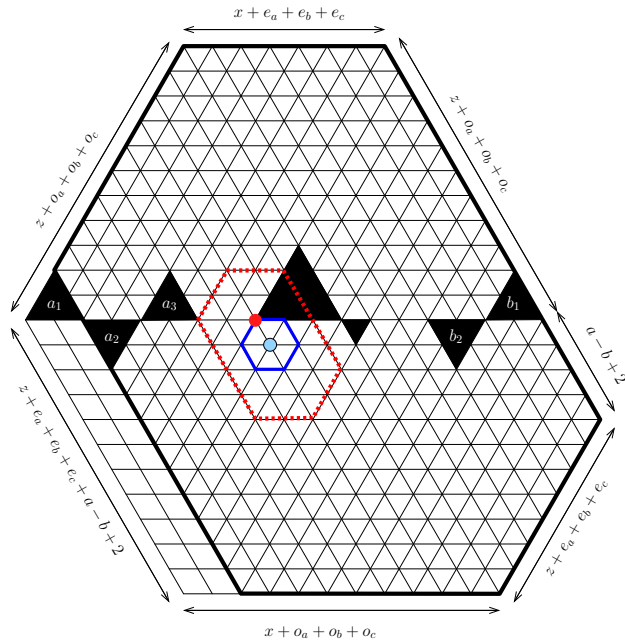

(a)

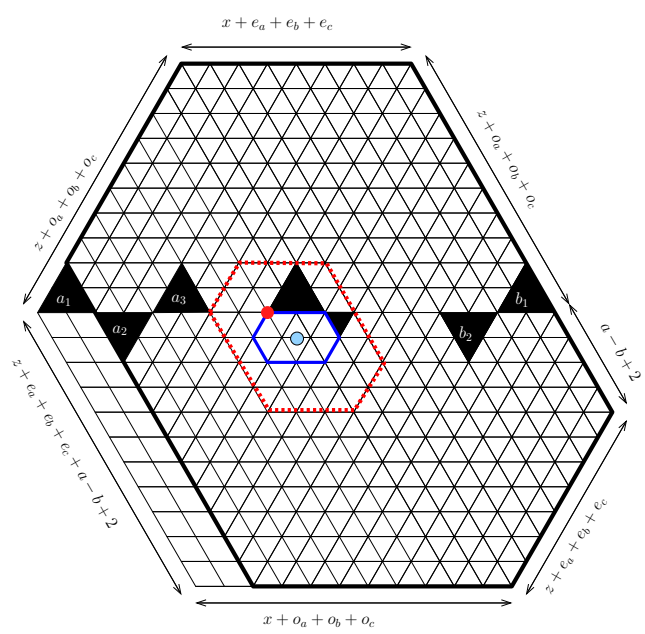

(b)

Figure 25: (a) Obtaining an $E^{(2)}$-type region from the region $\bar{E}_{x, 0, z}^{(2)}(\mathbf{a} ; \mathbf{c} ; \mathbf{b})$ with $a \geqslant b$. (b) Obtaining an $E^{(4)}$-type region from the region $\bar{E}_{x, 0, z}^{(4)}(\mathbf{a} ; \mathbf{c} ; \mathbf{b})$ with $a \geqslant b$.

Case 1b: $R$ is an upper region, and $0<2 d \leqslant b-a$.

We have, in this case, $y \geqslant-2 d$. When $y=-2 d$, we have

$$
\begin{aligned}
& \mathrm{M}\left(E_{x,-2 d, z}^{(i)}(\mathbf{a} ; \mathbf{c} ; \mathbf{b})\right)=\mathrm{M}\left(\bar{E}_{x, \min \left(b_{1}, b-a\right)-2 d, z}^{(i)}\left(\mathbf{a} ;{ }^{0} \mathbf{c} ; b_{2}, \ldots, b_{n}\right)\right), \\
& \mathrm{M}\left(F_{x,-2 d, z}^{(j)}(\mathbf{a} ; \mathbf{c} ; \mathbf{b})\right)=\mathrm{M}\left(\bar{F}_{x, \min \left(b_{1}, b-a\right)-2 d, z}^{(j)}\left(\mathbf{a} ;{ }^{0} \mathbf{c} ; b_{2}, \ldots, b_{n}\right)\right),
\end{aligned}
$$




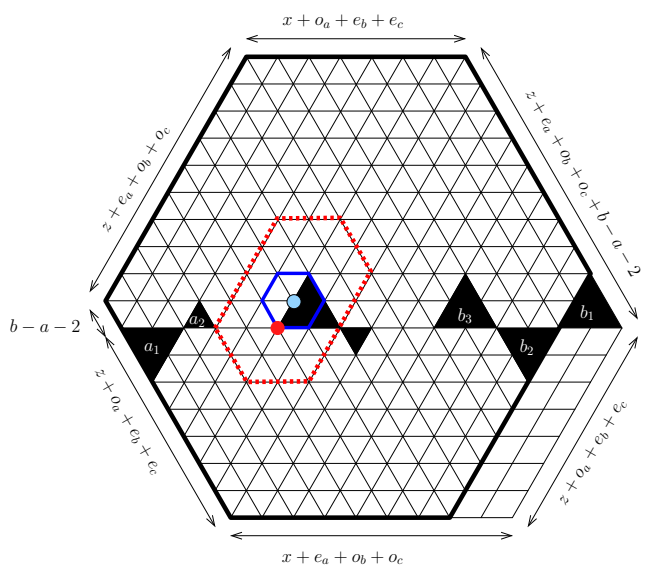

(a)

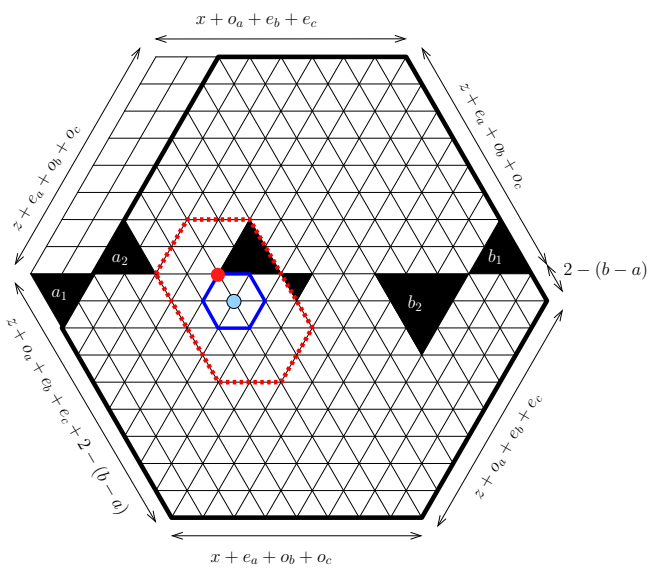

(b)

Figure 26: (a) Obtaining an (horizontally reflected) $\bar{E}^{(2)}$-type region from the region $E_{x,-2, z}^{(2)}(\mathbf{a} ; \mathbf{c} ; \mathbf{b})$ in the case $b-a \geqslant 2$. (b) Obtaining an $\bar{E}^{(2)}$-type region from the region $E_{x,-2, z}^{(2)}(\mathbf{a} ; \mathbf{c} ; \mathbf{b})$ in the case $0<b-a \leqslant 2$.

$$
\begin{aligned}
& \mathrm{M}\left(G_{x,-2 d, z}^{(k)}(\mathbf{a} ; \mathbf{c} ; \mathbf{b})\right)=\mathrm{M}\left(\bar{G}_{x, \min \left(b_{1}, b-a\right)-2 d, z}^{(k)}\left(\mathbf{a} ;{ }^{0} \mathbf{c} ; b_{2}, \ldots, b_{n}\right)\right), \\
& \mathrm{M}\left(K_{x,-2 d, z}^{(l)}(\mathbf{a} ; \mathbf{c} ; \mathbf{b})\right)=\mathrm{M}\left(\bar{K}_{x, \min \left(b_{1}, b-a\right)-2 d, z}^{(l)}\left(\mathbf{a} ;{ }^{0} \mathbf{c} ; b_{2}, \ldots, b_{n}\right)\right),
\end{aligned}
$$

for $i=2,3, j=4,5,6, k=2,3,4,5$, and $l=5,6,7,8$ (illustrated in Figure 26(a)). Recall that we use the notation ${ }^{0} \mathbf{c}$ for the sequence obtained from $\mathbf{c}$ by including a new 0 term in the front. It is easy to see that the regions on the right-hand sides of the above equalities satisfy the conditions of the lemma.

Case 1c: $R$ is an upper region, and $0<b-a \leqslant 2 d$.

We have here $y \geqslant a-b$. When $y=a-b$, we have

$$
\begin{aligned}
& \mathrm{M}\left(E_{x, a-b, z}^{(i)}(\mathbf{a} ; \mathbf{c} ; \mathbf{b})\right)=\mathrm{M}\left(\bar{E}_{x, a-b, z}^{(i)}\left(a_{2}, \ldots, a_{m} ; \mathbf{c} ; \mathbf{b}\right)\right), \\
& \mathrm{M}\left(F_{x,-2 d, z}^{(j)}(\mathbf{a} ; \mathbf{c} ; \mathbf{b})\right)=\mathrm{M}\left(\bar{F}_{x, a-b, z}^{(j)}\left(a_{2}, \ldots, a_{m} ; \mathbf{c} ; \mathbf{b}\right)\right), \\
& \mathrm{M}\left(G_{x,-2 d, z}^{(k)}(\mathbf{a} ; \mathbf{c} ; \mathbf{b})\right)=\mathrm{M}\left(\bar{G}_{x, a-b, z}^{(k)}\left(a_{2}, \ldots, a_{m} ; \mathbf{c} ; \mathbf{b}\right)\right), \\
& \mathrm{M}\left(K_{x,-2 d, z}^{(l)}(\mathbf{a} ; \mathbf{c} ; \mathbf{b})\right)=\mathrm{M}\left(\bar{K}_{x, a-b, z}^{(l)}\left(a_{2}, \ldots, a_{m} ; \mathbf{c} ; \mathbf{b}\right)\right),
\end{aligned}
$$

for $i=2,3, j=4,5,6, k=2,3,4,5$, and $l=5,6,7,8$ (see Figure 26(b) for an example). It is easy to see that the regions on the right-hand sides of the above equalities satisfy the conditions of the lemma.

Case 2a: $R$ is a lower region and $a \leqslant b$.

We have $y \geqslant 0$. When $y=0$, we get by removing forced lozenges:

$$
\mathrm{M}\left(E_{x, 0, z}^{(i)}(\mathbf{a} ; \mathbf{c} ; \mathbf{b})\right)=\mathrm{M}\left(\bar{E}_{x, \min \left(b_{1}, b-a\right), z}^{(3+i \bmod 6)}\left(b_{2}, \ldots, b_{n} ; \overline{\mathbf{c}} ; \mathbf{a}\right)\right),
$$




$$
\begin{aligned}
& \mathrm{M}\left(F_{x, 0, z}^{(j)}(\mathbf{a} ; \mathbf{c} ; \mathbf{b})\right)=\mathrm{M}\left(\bar{F}_{x, \min \left(b_{1}, b-a\right), z}^{(4+j \bmod 8)}\left(b_{2}, \ldots, b_{n} ; \overline{\mathbf{c}} ; \mathbf{a}\right)\right) \\
& \mathrm{M}\left(G_{x, 0, z}^{(k)}(\mathbf{a} ; \mathbf{c} ; \mathbf{b})\right)=\mathrm{M}\left(\bar{G}_{x, \min \left(b_{1}, b-a\right), z}^{(4+k} \bmod \left(b_{2}, \ldots, b_{n} ; \overline{\mathbf{c}} ; \mathbf{a}\right)\right) \\
& \mathrm{M}\left(K_{x, 0, z}^{(l)}(\mathbf{a} ; \mathbf{c} ; \mathbf{b})\right)=\mathrm{M}\left(\bar{K}_{x, \min \left(b_{1}, b-a\right), z}^{(4+l)}\left(b_{2}, \ldots, b_{n} ; \overline{\mathbf{c}} ; \mathbf{a}\right)\right)
\end{aligned}
$$

for $i=1,4,5,6, j=1,2,3,7,8, k=1,6,7,8$, and $l=1,2,3,4$. Recall that $\overline{\mathbf{c}}$ is the sequence obtained from $\mathbf{c}$ by reverting the order of the terms if we have an even number of terms, otherwise, we revert the sequence and include a new 0 term in front of the resulting one. One readily sees that the regions on the right-hand sides of the above equalities satisfy the conditions of the lemma.

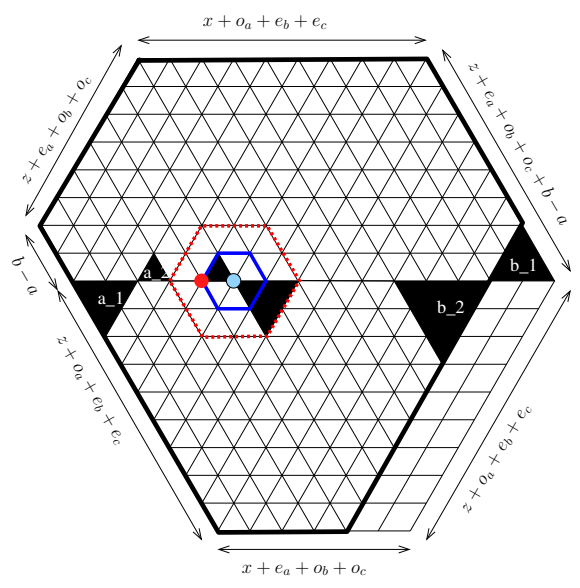

(a)

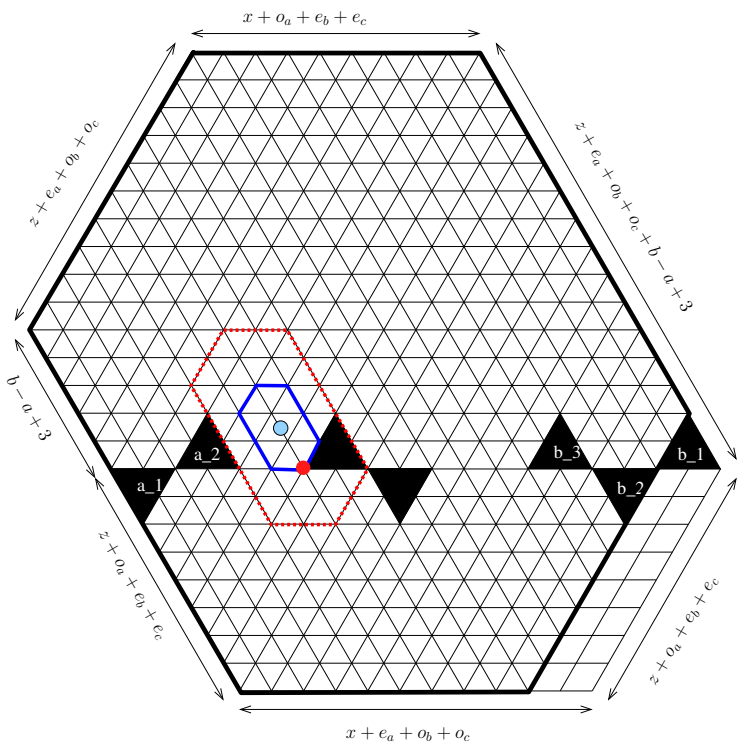

(b)

Figure 27: (a) Obtaining an $\bar{E}^{(1)}$-type region from the region $E_{x, 0, z}^{(1)}(\mathbf{a} ; \mathbf{c} ; \mathbf{b})$ with $a \leqslant b$. (b) Obtaining an $\bar{K}^{(6)}$-type region from the region $K_{x, 0, z}^{(2)}(\mathbf{a} ; \mathbf{c} ; \mathbf{b})$ with $a \leqslant b$.

Case 2b: $R$ is a lower region and $0<2 d \leqslant a-b$.

We have $y \geqslant-2 d$. If $y=-2 d$, then by investigating forced lozenges, we get

$$
\begin{aligned}
& \mathrm{M}\left(E_{x, a-b, z}^{(i)}(\mathbf{a} ; \mathbf{c} ; \mathbf{b})\right)=\mathrm{M}\left(\bar{E}_{x, \min \left(a_{1}, a-b\right)-2 d, z}^{(3+i \bmod 6)}\left(a_{2}, \ldots, a_{m} ; \mathbf{c} ; \mathbf{b}\right)\right), \\
& \mathrm{M}\left(F_{x,-2 d, z}^{(j)}(\mathbf{a} ; \mathbf{c} ; \mathbf{b})\right)=\mathrm{M}\left(\bar{F}_{x, \min \left(a_{1}, a-b\right)-2 d, z}^{(4+j}\left(a_{2}, \ldots, a_{m} ; \mathbf{c} ; \mathbf{b}\right)\right), \\
& \mathrm{M}\left(G_{x,-2 d, z}^{(k)}(\mathbf{a} ; \mathbf{c} ; \mathbf{b})\right)=\mathrm{M}\left(\bar{G}_{x, \min \left(a_{1}, a-b\right)-2 d, z}^{(4+k}\left(a_{2}, \ldots, a_{m} ; \mathbf{c} ; \mathbf{b}\right)\right), \\
& \mathrm{M}\left(K_{x,-2 d, z}^{(l)}(\mathbf{a} ; \mathbf{c} ; \mathbf{b})\right)=\mathrm{M}\left(\bar{K}_{x, \min \left(a_{1}, a-b\right)-2 d, z}^{(4+l \bmod 8)}\left(a_{2}, \ldots, a_{m} ; \mathbf{c} ; \mathbf{b}\right)\right),
\end{aligned}
$$

for $i=5,6, j=1,2,8, k=1,6,7,8$, and $l=1,2,3,4$ (see Figure 28(a) for an example). Again, we can check that the new regions (the ones on the right-hand sides of the above 


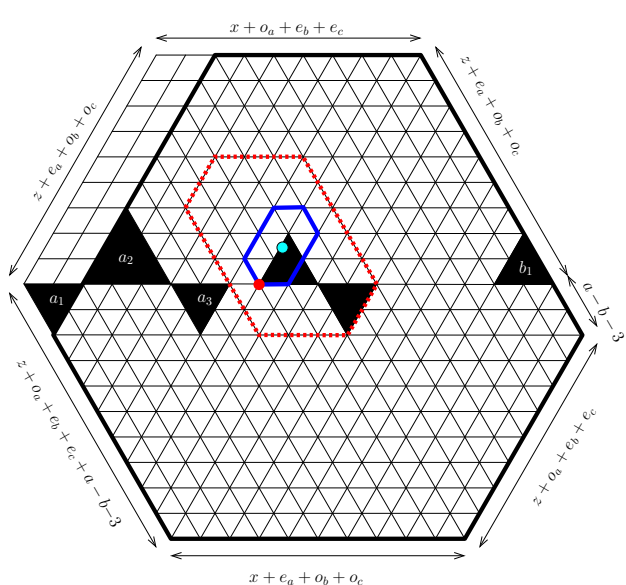

(a)

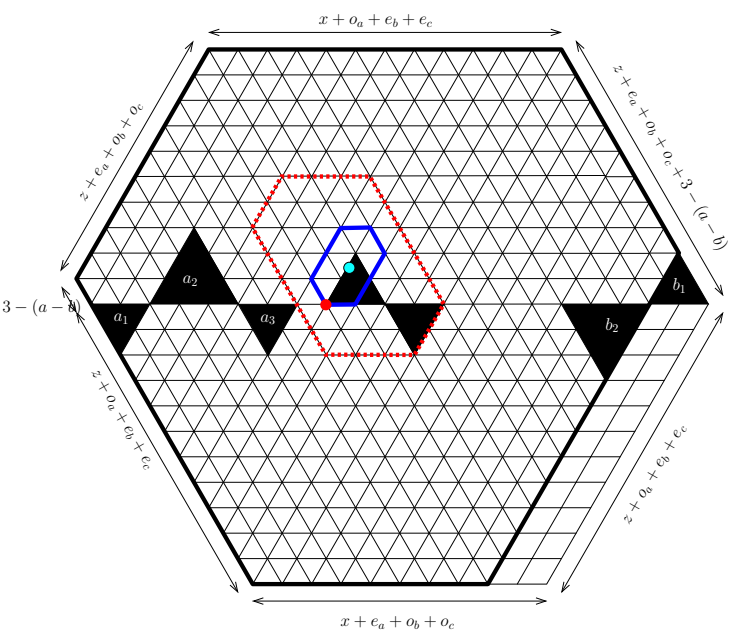

(b)

Figure 28: (a) Obtaining an $\bar{K}^{(3)}$-type region from the region $K_{x,-3, z}^{(3)}(\mathbf{a} ; \mathbf{c} ; \mathbf{b})$ with $a-b \geqslant 3$. (b) Obtaining an (horizontally reflected) $\bar{K}^{(7)}$-type region from the region $K_{x, a-b, z}^{(3)}(\mathbf{a} ; \mathbf{c} ; \mathbf{b})$ with $0<a-b \leqslant 3$.

identities) have the $h$-parameter strictly less than that of the corresponding regions on the left-hand sides.

Case 2c: $R$ is a lower region and $0<a-b \leqslant 2 d$.

We have $y \geqslant b-a$ (in this case $b-a$ is negative). If $y=b-a$, then

$$
\begin{aligned}
& \mathrm{M}\left(E_{x, a-b, z}^{(i)}(\mathbf{a} ; \mathbf{c} ; \mathbf{b})\right)=\mathrm{M}\left(\bar{E}_{x, b-a, z}^{(3+i \bmod 6)}\left(\mathbf{a} ;{ }^{0} \mathbf{c} ; b_{2}, \ldots, b_{n}\right)\right) \\
& \mathrm{M}\left(F_{x,-2 d, z}^{(j)}(\mathbf{a} ; \mathbf{c} ; \mathbf{b})\right)=\mathrm{M}\left(\bar{F}_{x, b-a, z}^{(4+j \bmod 8)}\left(\mathbf{a} ;{ }^{0} \mathbf{c} ; b_{2}, \ldots, b_{n}\right)\right), \\
& \mathrm{M}\left(G_{x,-2 d, z}^{(k)}(\mathbf{a} ; \mathbf{c} ; \mathbf{b})\right)=\mathrm{M}\left(\bar{G}_{x, b-a, z}^{(4+k \bmod 8)}\left(\mathbf{a} ;{ }^{0} \mathbf{c} ; b_{2}, \ldots, b_{n}\right)\right), \\
& \mathrm{M}\left(K_{x,-2 d, z}^{(l)}(\mathbf{a} ; \mathbf{c} ; \mathbf{b})\right)=\mathrm{M}\left(\bar{K}_{x, b-a, z}^{(4+l \bmod 8)}\left(\mathbf{a} ;{ }^{0} \mathbf{c} ; b_{2}, \ldots, b_{n}\right)\right),
\end{aligned}
$$

for $i=5,6, j=1,2,8, k=1,6,7,8$, and $l=1,2,3,4$ (illustrated in Figure 28(b)). One readily sees that the regions on the right-hand sides of the above equalities satisfy the conditions of the lemma. This finishes our proof.

\subsection{Combined proof for the tiling formulas of all off-central regions}

We simultaneously prove all 30 tiling formulas in Section 2 by induction on $h=p+x+z$. Recall that $p$ denotes the quasi-perimeter of the region, i.e., the perimeter of the base hexagon, and that our region is obtained by removing three ferns from the base hexagon.

By Lemma 49, we can assume that our three ferns consist of all triangles of positive side-lengths, except for a possible 0 -triangle in front of the middle fern.

The base cases are $x=0, z=0$, and $p<6$. 


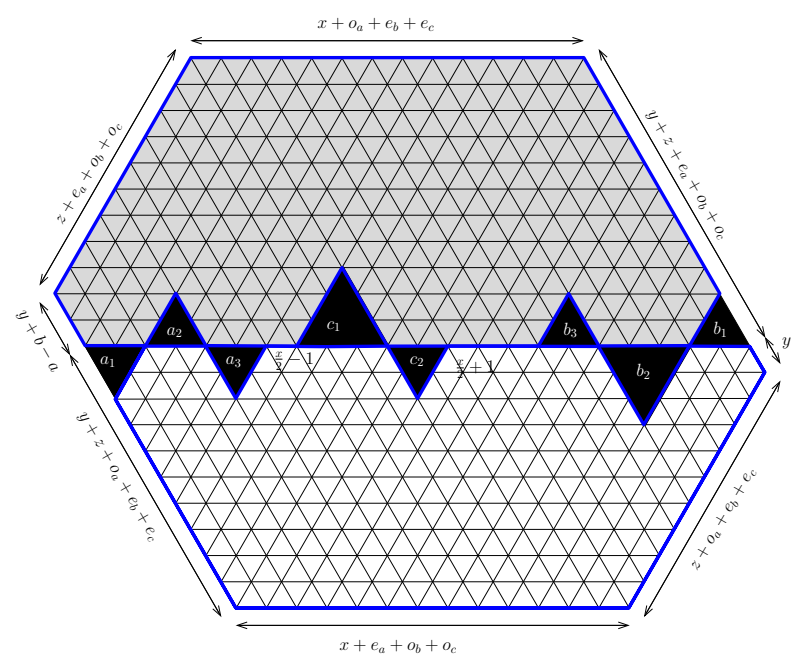

Figure 29: Dividing the region $E_{x, y, 0}^{(1)}(\mathbf{a} ; \mathbf{c} ; \mathbf{b})$ in to two dented semihexagons.

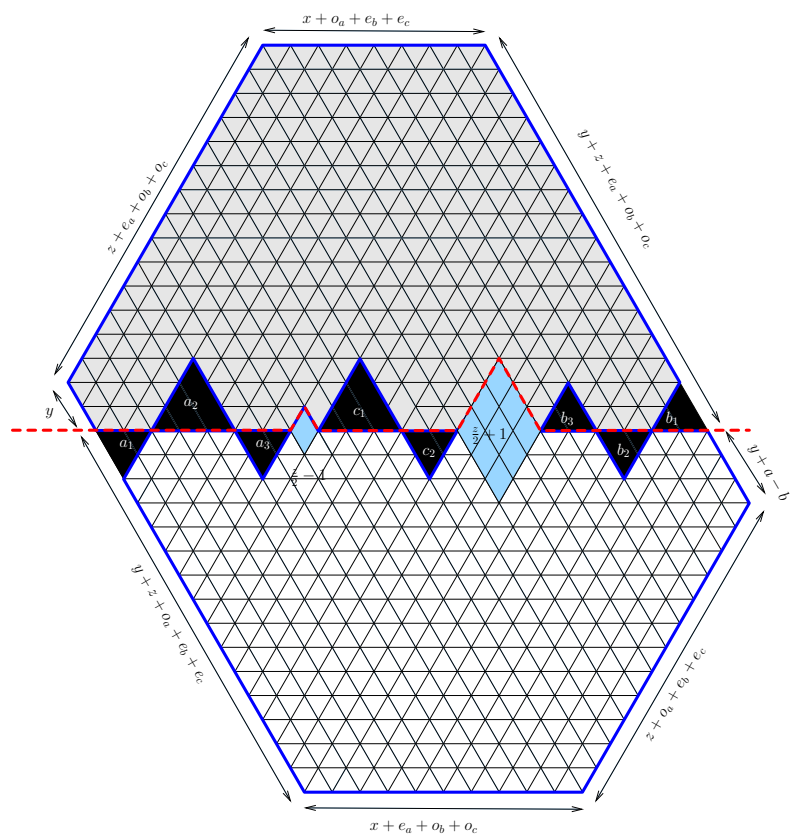

Figure 30: Dividing the region $E_{0, y, z}^{(1)}(\mathbf{a} ; \mathbf{c} ; \mathbf{b})$ in to two dented semihexagons.

If $z=0$, we divide our region into two dented semihexagons with dents by cutting along the horizontal lattice line containing our three ferns. These semihexagons correspond to the two s-terms in our tiling formulas (shown in Figure 29). By the Region-splitting Lemma 46, the tiling number of our region is equal to the product of tiling numbers of the two dented semihexagons. Then our theorems follow from Cohn-Larsen-Propp's formula (5).

If $x=0$, we also partition the region into two parts along the same lattice line above; the only difference is that we now add two 'bumps' of the same size as the gaps between 
the three ferns. The upper part is a dented semihexagon, and the lower part (after removing several vertical forced lozenges at the two bumps) is congruent with another dented semihexagon (illustrated in Figure 30). Again, the theorems follow from CohnLarsen-Propp's formula (5) and the Region-splitting Lemma 46.

If $p<6$, by Lemma 45 , we have $2 x+4 z<6$. It implies that at least one of $x$ and $z$ is 0 . This base case is then reduced to the above base cases.

For the induction step, we assume that $x$ and $z$ are positive, that $p \geqslant 6$, and that all our 30 tiling formulas hold for off-central regions whose $h$-parameter is strictly less than $h=p+x+z$.

If $y$ does not achieve its minimal value, by the 66 recurrences in Sections 3.3-3.10, the tiling number of each of our off-central regions can be written in terms of tiling numbers of other regions, that are either the central case treated in [31, Theorems 2-9] or other off-central regions with a smaller $h$-parameter. Its means that by Theorems $2-9$ in [31] and the induction hypothesis, we get an explicit formula for the tiling number of each of our off-central regions. Then, by performing a straightforward simplification, one can verify that the latter explicit tiling formula agrees with our theorem.

If $y$ achieves its minimal value, our 66 recurrences do not work anymore. However, by Lemma 50, each of our regions has the same tiling number as a new off-central region with a smaller $h$-parameter. Thus, our theorems again follow the induction hypothesis. This finishes our proof.

\section{Acknowledgement}

The author would like to thank Dennis Stanton and Hjalmar Rosengren for pointing out to him paper [43]. The author also thanks to anonymous reviewers for carefully reading the original version of the paper and giving helpful comments.

\section{References}

[1] F. Abeles, Dodgson condensation: The historical and mathematical development of an experimental method, Linear Algebra Appl. 429 (2-3) (2008), 429-438

[2] G. E. Andrews, Plane partitions (III): The weak Macdonald conjecture, Invent. Math., 53 (1979), 193-225.

[3] M. Ciucu, Enumeration of lozenge tilings of punctured hexagons, J. Combin. Theory Ser. A, 83 (1998), 268-272.

[4] M. Ciucu, Plane partition I: A generalization of MacMahon's formula, Memoirs of Amer. Math. Soc., 178 (2005), no. 839, 107-144.

[5] M. Ciucu, A generalization of Kuo condensation, J. Combin. Theory Ser. A, 134 (2015), 221-241.

[6] M. Ciucu, Another dual of MacMahon's theorem on plane partitions, Adv. Math., 306 (2017), 427-450. 
[7] M. Ciucu, T. Eisenkölbl, C. Krattenthaler, and D. Zare, Enumeration of lozenge tilings of hexagons with a central triangular hole, J. Combin. Theory Ser. A, 95 (2001), 251-334.

[8] M. Ciucu and I. Fischer, Lozenge tilings of hexagons with arbitrary dents, Adv. Appl. Math., 73 (2016), 1-22.

[9] M. Ciucu and C. Kattenthaler, The number of centered lozenge tilings of a symmetric hexagon, J. Combin. Theory Ser. A, 86 (1999), 103-126.

[10] M. Ciucu and C. Krattenthaler, A dual of MacMahon's theorem on plane partitions, Proc. Natl. Acad. Sci. USA, 110 (2013), 4518-4523.

[11] M. Ciucu and T. Lai, Lozenge tilings doubly-intruded hexagons, J. Combin. Theory Ser. A, 167 (2019), 294-339.

[12] H. Cohn, M. Larsen, and J. Propp, The shape of a typical boxed plane partition, New York J. Math., 4 (1998), 137-165.

[13] C.L. Dodgson, Condensation of determinants, Proc. Roy. Soc. London, 15 (1866), $150-155$.

[14] M. Fulmek, Graphical Condensation, Overlapping Pfaffians and Superpositions of Matchings, Electron. J. Combin., 17(1) (2010), \#R83.

[15] T. Eisenkölbl, Rhombus tilings of a hexagon with three fixed border tiles, J. Combin. Theory Ser. A, 88 (2) (1999), 368-378.

[16] H. Helfgott and I. Gessel, Tilings of diamonds and hexagons with defects, Electron. J. Combin., 6 (1999), \#R16.

[17] R. Kenyon and D. Wilson, The space of circular planar electrical networks, SIAM J. Discrete Math., 31(1), 1-28.

[18] D.E. Knuth, Overlapping pfaffians, Electron. J. Combin., 3 (1996), R5.

[19] C. Koutschan, M. Kauer, and Zeilberger, A proof of George Andrews' and David Robbins' a TSPP-conjecture. Proc. Natl. Acad. Sci. USA, 108 (2011), 2196-2199.

[20] C. Krattenthaler, Plane partitions in the work of Richard Stanley and his school, "The Mathematical Legacy of Richard P. Stanley" P. Hersh, T. Lam, P. Pylyavskyy and V. Reiner (eds.), Amer. Math. Soc., R.I., 2016, pp. 246-277.

[21] E. H. Kuo, Applications of graphical condensation for enumerating matchings and tilings, Theor. Comput. Sci., 319 (2004), 29-57.

[22] E. H. Kuo, Graphical Condensation Generalizations Involving Pfaffians and Determinants, ArXiv Mathematics e-prints, May 2006. arXiv:math/0605154.

[23] G. Kuperberg, Symmetries of plane partitions and the permanent-determinant method, J. Comnin. Theory Ser. A, 68 (1994), 115-151.

[24] M. Leoni, G. Musiker, S. Neel, and P. Turner, Aztec Castles and the dP3 Quiver, J. Phys. A: Math. Theor., 47 (2014), \#474011.

[25] T. Lai, A generalization of Aztec dragons, Graph Combin., 2(5) (2016), 1979-1999. 
[26] T. Lai, A q-enumeration of lozenge tilings of a hexagon with three dents, Adv. Applied Math., 82 (2017), 23-57.

[27] T. Lai, A q-enumeration of a hexagon with four adjacent triangles removed from the boundary, European J. Combin., 64 (2017), 66-87.

[28] T. Lai, Lozenge Tilings of a Halved Hexagon with an Array of Triangles Removed from the Boundary, SIAM J. Discrete Math., 32(1) (2018), 783-814.

[29] T. Lai, Lozenge Tilings of a Halved Hexagon with an Array of Triangles Removed from the Boundary, Part II, Electron. J. Combin., 25(4) (2018), \#P4.58.

[30] T. Lai, Tiling Enumeration of Doubly-intruded Halved Hexagons, ArXiv Mathematics e-prints, Jan 2018. arXiv: 1801.00249

[31] T. Lai, Lozenge Tilings of Hexagons with Central Holes and Dents, Electron. J. Combin., 27(1) (2020), \#P1.61.

[32] T. Lai, Proof of a Conjecture of Kenyon and Wilson on Semicontiguous Minors, J. Combin. Theory Ser. A, 116 (2019), 134-163.

[33] T. Lai and G. Musiker, Beyond Aztec castles: Toric cascades in the dP3 quiver, Comm. Math. Phys. , 356(3) (2017), 823-88.

[34] T. Lai and R. Rohatgi, Cyclically Symmetric Tilings of a Hexagon with Four Holes, Adv. Appl. Math., 96 (2018), 249-285.

[35] P. A. MacMahon, Memoir on the theory of the partition of numbers-Part V. Partition in two-dimensional space, Phil. Trans. R. S., 1911, A.

[36] T. Muir, The Theory of Determinants in the Historical Order of Development, Vol. I, Macmillan, London, 1906.

[37] S. Okada and C. Krattenthaler, The number of rhombus tilings of a 'punctured' hexagon and the minor summation formula, Adv. in Appl. Math.,21 (1998), 381404.

[38] J. Propp, Enumeration of matchings: Problems and progress, New Perspectives in Geometric Combinatorics, Cambridge Univ. Press, Cambridge, 1999, 255-291.

[39] J. Propp, Generalized domino-shuffling, Theoret. Comput. Sci., 303 (2003), 267-301.

[40] R. Proctor, Odd symplectic groups, Invent. Math, 92(2) (1988), 307-332.

[41] R. Rohatgi, Enumeration of lozenge tilings of halved hexagons with a boundary defect, Electron. J. Combin., 22(4) (2015), P4.22.

[42] R. Rohatgi, Enumeration of tilings of a hexagon with a maximal staircase and a unit triangle removed, Australas. J. Combin., 65(3) (2016), 220-231.

[43] H. Rosengren, Selberg integrals, Askey-Wilson polynomials and lozenge tilings of a hexagon with a triangular hole, J. Combin. Theory Ser. A 138 (2016), 29-59.

[44] D. E Speyer, Perfect Matchings and the Octahedron Recurrence, J. Algebraic Combin., 25(6) (2007), 309-348.

[45] R. Stanley, Symmetries of plane partitions, J. Combin. Theory Ser. A, 43 (1986), $103-243$. 
[46] J. R. Stembridge, The enumeration of totally symmetric plane partitions, Adv. in Math., 111 (1995), 227-243.

[47] W. Yan, Y. Yeh, and F. Zhang, Graphical condensation of plane graphs: A combinatorial approach, Theoret. Comput. Sci., 349(3) (2005), 452-461.

[48] W. Yan and F. Zhang, Graphical condensation for enumerating perfect matchings, J. Combin. Theory Ser. A, 110 (2005), 113-125.

[49] D. Zeilberger, Dodgson's Determinant-Evaluation Rule proved by Two-Timing Men and Women, Electron. J. Combin., 4(2)(1997),\#R22. 